\title{
Probabilistic Analysis of a Thin-walled Beam with a Crack
}

\author{
Chalitphan Kunaporn
}

Dissertation submitted to the faculty of the Virginia Polytechnic Institute and State University in partial fulfillment of the requirements for the degree of

\author{
Doctor of Philosophy \\ In \\ Engineering Mechanics
}

\author{
Mahendra P. Singh \\ Surot Thangjitham \\ Mayuresh J. Patil \\ Rakesh K. Kapania \\ Saad A. Ragab \\ Scott W. Case
}

January 21, 2010

Blacksburg, Virginia

Keywords: Cracked beam, Thin-walled, Reliability method, Crack propagation, Divergence 


\title{
Probabilistic Analysis of a Thin-walled Beam with a Crack
}

Chalitphan Kunaporn

\begin{abstract}
(ABSTRACT)
It is reasonable to assume that an aircraft might experience some in-flight discrete source damage caused by various incidents. It is, thus, necessary to evaluate the impact of such damage on the performance of the aircraft. This study is focused on evaluating the effect of a simple discrete damage in an aircraft wing on its static and dynamic response. The damaged wing is modeled by a thin-walled beam with a longitudinal crack the response of which can be obtained analytically. As uncertainties are present in the location and size of the crack as well as in the applied loads, their effects are incorporated into the framework consisting of structural response, crack propagation and aeroelasticity.
\end{abstract}

The first objective of this study is to examine the effect of damage represented by a crack on the wing flexibility that influences its deformation and aero-elastic divergence characteristics. To study this, the thin-walled beam is modeled by Benscoter thin-walled beam theory combined with Gunnlaugsson and Pedersen compatibility conditions to accurately account for the discontinuity at the interface of the cracked and uncracked beam segments. Instead of conducting a detailed finite element analysis, the solution is obtained in an exact sense for general distributed loads representing the wind pressure effects. This analytical approach is shown to provide very accurate values for the global beam response compared with the detailed finite element shell analysis. This analytical solution is, then, used to study the beam response probabilistically. The crack location and size are assumed to be uncertain and are, thus, characterized by random variable. For a specified limit state, the probability of failure can be conveniently calculated by the first order second moment analysis using the safety index approach. The same analytical solution is also used to study the aero-elastic divergence characteristics of a wing, the inner structure of which is represented by a thin-walled beam with a crack of uncertain size and position along the beam. 
The second objective of this study is to examine the time growth of a crack under dynamic gust type of loading to which a wing is likely to be exposed during flight. Damage propagating during operation further deteriorates the safety of the aircraft and it is necessary to study its time growth so that its impact on the performance can be evaluated before it reaches its unstable state. The proposed framework for the crack growth analysis is based on classical fracture mechanics where the remaining flight time is obtained by Monte Carlo simulation in which various uncertainties are taken into account. To obtain equivalent cyclic loading required for crack growth analysis, random vibration analysis of the thin-walled beam is conducted for stochastic wind load defined by a gust load spectral density function. The probability of failure represented by the crack size approaching the critical crack size within the flight duration or the remaining flight time before a crack reaches its limiting value are obtained.

This study with a simple representation of a wing and damage is anticipated to provide initial guidance for future studies to examine the impact of discrete source damage on the in-flight performance of the aircrafts, with the ultimate goal of minimizing the adverse effect and enhancing the safety of aircrafts experiencing damage. 


\section{Acknowledgements}

I gratefully acknowledge the encouragement, guidance and support from my advisor Professor Mahendra Singh, my co-advisor Professor Surot Thangjitham and my research project supervisors Professor Mayuresh Patil and Professor Rakesh Kapania. I am also thankful to my other committee members, Professor Saad Ragab and Professor Scott Case for comments and suggestions to improve this research.

This research was conducted with financial support from National Aeronautics and Space Administration, IRAC program (Agreement \#NNX08AC49A) under the technical monitoring by Dr. Thiagaraja Krishnamurthy of NASA Langley Research Center. The support is gratefully acknowledged. I also thank to all other IRAC group students for their friendships and technical support during the project. I am also indebted to the faculty and staff of the department of engineering science and mechanics for continuing supports throughout this degree.

My deepest gratitude belongs to my mom, my dad and my sister for their endless love. Special thanks to my uncles, my aunts, my cousins and my friends for their encouragement. 
To my beloved mom, dad and sister 


\section{Contents}

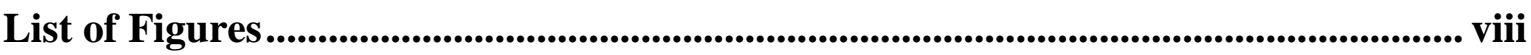

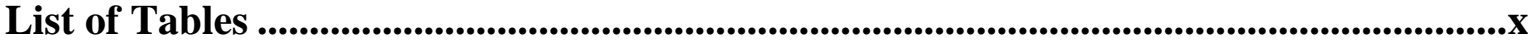

Chapter 1: Introduction .................................................................................................1

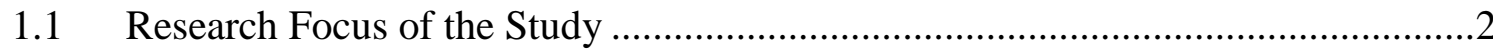

1.1.1 Modeling of Thin-walled Beams ...............................................................4

1.1.2 Probabilistic Methods and Reliability Analysis ..........................................

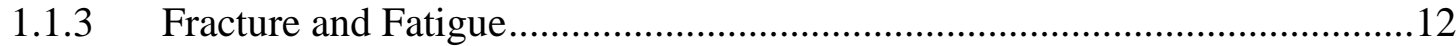

1.2 Dissertation Organization.......................................................................... 13

Chapter 2: $\quad$ Probabilistic Analysis of a Thin-walled Beam with a Crack.................16

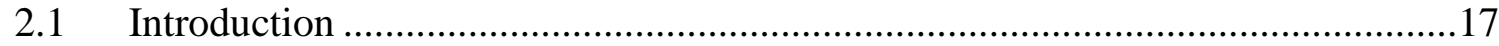

2.2 Analytical Model of a Thin-walled Beam with a Crack...................................18

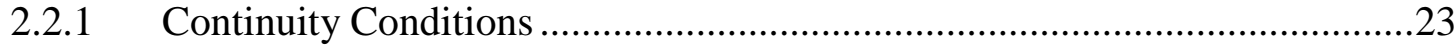

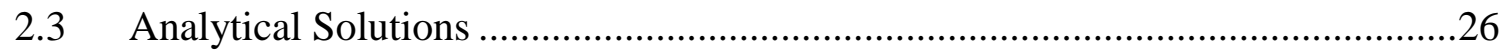

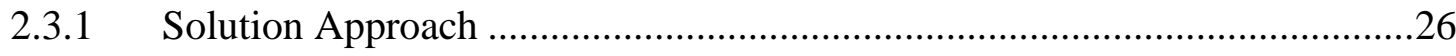

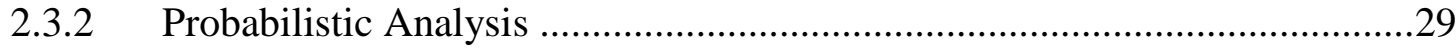

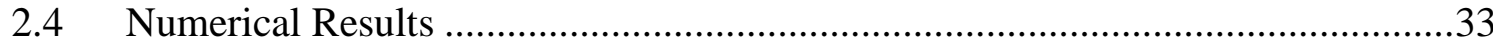

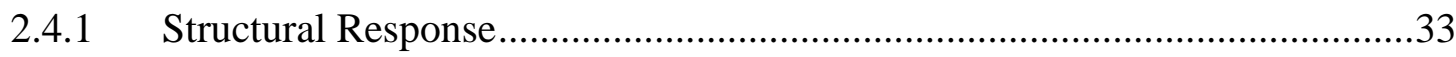

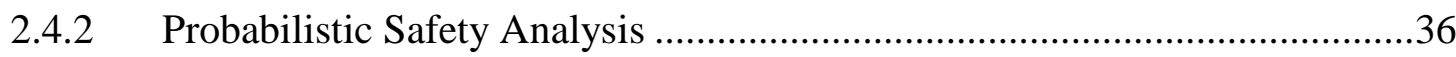

2.5 Summary and Concluding Remarks ............................................................41

Chapter 3: $\quad$ Random Crack Propagation of a Thin-walled Beam with a Crack.....42

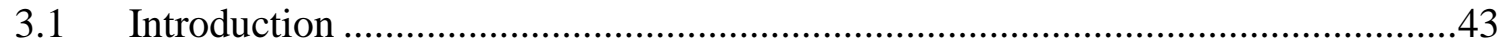

3.2 Crack Growth Modeling .........................................................................45

3.3 Dynamic Analysis of a Thin-walled Beam …............................................47

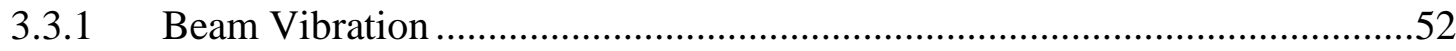

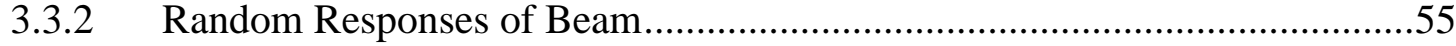

3.3.3 Stochastic Characteristics of Responses .................................................59

3.4 Crack Growth Simulations under Stochastic Loadings .....................................61 


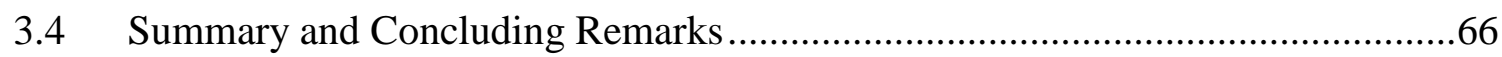

Chapter 4: Aeroelastic Divergence of a Thin-walled Beam with a Crack...............67

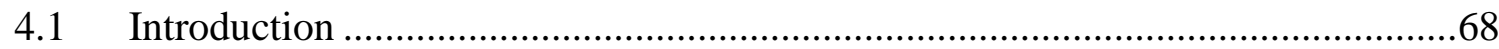

4.2 Aeroelastic Modeling of a Wing as a Cracked Thin-walled Beam ........................70

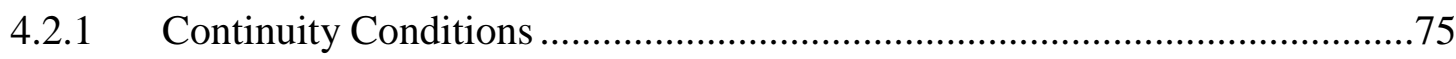

4.2.2 Aerodynamic Loadings and Divergence ………….......................................78

4.3 Analytical Solutions …………………………...............................................

4.3.1 Pressure at Divergence: Iterative Approach....................................................83

4.3.2 Pressure at Divergence: Direct Approach ....................................................85

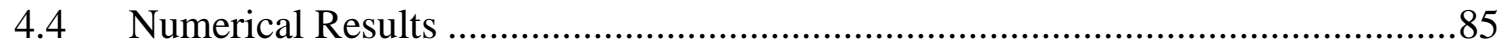

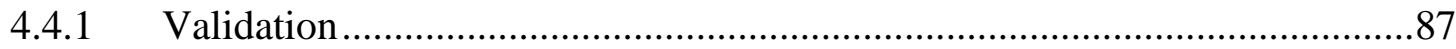

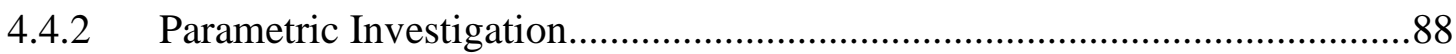

4.4.3 Probabilistic Characteristics of the Divergence Dynamic Pressure ................90

4.5 Summary and Concluding Remarks .................................................................97

Chapter 5: Summary and Concluding Remarks ...............................................................98

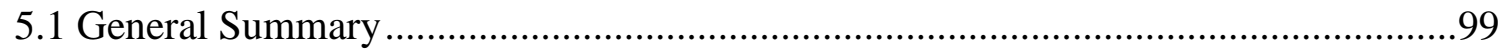

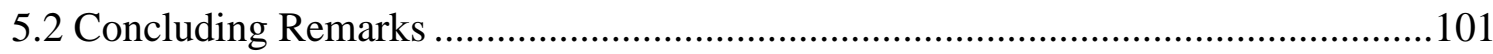

5.3 Suggestions for Future Research ......................................................................... 101

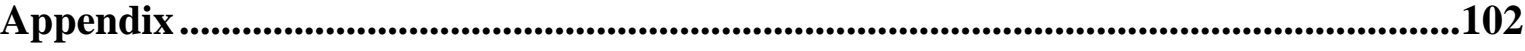

Appendix A Numerical Examples of Jordan Canonical Form ........................................103

Appendix B Assembled Matrix Components..................................................................107

Appendix C Configurations of Shell Model used to validate the results obtained by

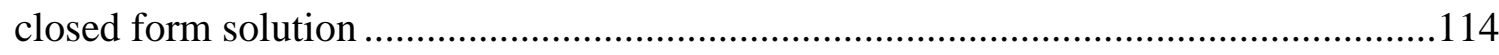

References ...............................................................................................................................................115 


\section{List of Figures}

Figure 1.1 Free body diagram of a flexural-torsional member ......................................... 5

Figure 2.1 Damaged structure modeled with a cracked thin-walled cantilever......................... 19

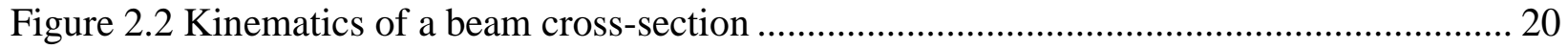

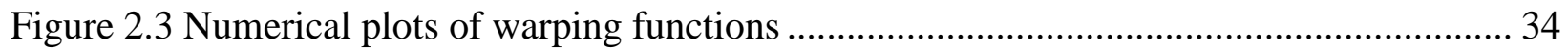

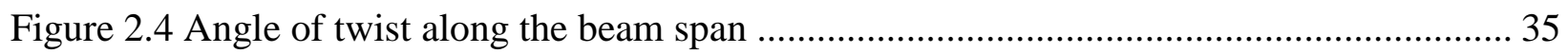

Figure 2.5 Lateral deflection along the beam span ............................................................ 35

Figure 2.6 Distributed twisting moment along the beam span ........................................... 36

Figure 2.7 Plots of safety indices and probabilities of failure versus coefficient of variation for $\alpha$ $=0.3$, quarter-ellipse loading and different mean crack sizes ........................................... 38

Figure 2.8 Plots of safety indices and probabilities of failure versus coefficient of variation for mean crack sizes of $15 \%, \alpha=0.3$ and three different loadings .......................................... 39 Figure 2.9 Plots of safety indices and probabilities of failure versus coefficient of variation for mean crack sizes of $20 \%, \alpha=0.3$ and three different loadings 39 Figure 2.10 Plots of safety indices and probabilities of failure versus location of crack for mean crack sizes of $15 \%$, coefficient of variation of $20 \%, \alpha=0.3$ and three different loadings ......

Figure 2.11 Plots of safety indices and probabilities of failure versus location of crack for coefficient of variation of $20 \%, \alpha=0.3$, quarter-ellipse loading and two different mean crack

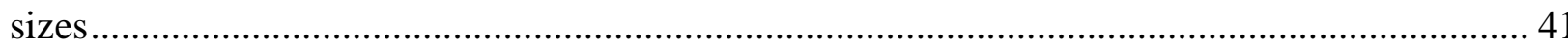

Figure 3.1 Cracked wing model based on a thin-walled cantilever ...................................... 48

Figure 3.2 Kinematics of a beam cross-section ............................................................ 48

Figure 3.3 Power spectral density of the twisting distributed moment (Input) .........................58

Figure 3.4 Power spectral density of the shear stress at the mid span (Output) ........................ 58

Figure 3.5 Comparison of the calculated probability density function of the peaks of the shear

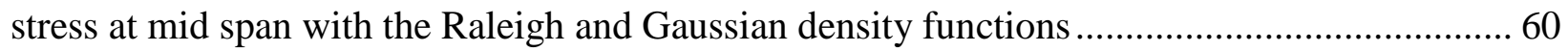
Figure 3.6 Root mean square values of dynamic shear stresses at various locations along the span

Figure 3.7 Evolution of crack growth with time for three damage locations, $L / 4$ (left), $L / 2$ (middle) and $3 L / 4$ (right) from the fixed end. 
Figure 3.8 Histograms of the crack length distribution at three time instants of 1 hour (left), 2 hours (middle) and 3 hours (right) for three damage locations from the fixed end..... 65 Figure 3.9 Critical crack length distribution for three damage locations, $L / 4$ (left), $L / 2$ (middle) and $3 L / 4$ (right) from the fixed end. 65 Figure 3.10 Time evolution of the probability of failure for three damage locations, $L / 4$ (left), $L / 2$ (middle) and $3 L / 4$ (right) from the fixed end.... 66

Figure 4.1 Damaged structure modeled with a cracked thin-walled cantilever......................... 70

Figure 4.2 Kinematics of a beam cross-section ............................................................. 71

Figure 4.3 Airfoil as a shell around the thin-walled beam wing structure................................ 78

Figure 4.4 Configurations of an airfoil showing the angle of incident and the angle of twist ..... 79

Figure 4.5 Numerical plots of warping functions used in the study. ..................................... 86

Figure 4.6 Divergence dynamic pressures for different crack sizes located at three different positions along the beam. 89

Figure 4.7 Normalized divergence dynamic pressures for different crack sizes located at three different positions along the beam 89 Figure 4.8 Divergence dynamic pressures for three different size cracks located at different locations along the beam. 90 Figure 4.9 Distributions of divergence dynamic pressures for the crack location, $L_{c}: N(1.5,0.375)$ and three different distributions of crack sizes

Figure 4.10 Distributions of divergence dynamic pressures for the crack location, $L c$ :

$N(3.0,0.375)$ and three different distributions of crack sizes 92

Figure 4.11 Distributions of divergence dynamic pressures for the crack location, $L c$ :

$N(4.5,0.375)$ and three different distributions of crack sizes 93

Figure 4.12 Probability of divergence dynamic pressure for cracked beam being less than a factor time the divergence dynamic pressure of uncracked beam for three different coefficients of variation of crack size 96 


\section{List of Tables}

Table 2.1 Final converged values of design points and safety index values for a crack with

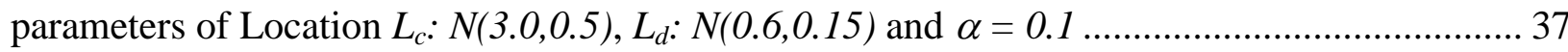
Table 2.2 Comparison of probability of failure values calculated by Rackwitz-Fiessler algorithm

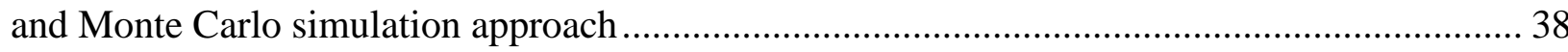

Table 3.1 Comparison of natural frequencies $(\mathrm{Hz})$ of a rectangular thin-walled cantilever........ 55

Table 3.2 Statistical parameters of shear stresses at different locations .................................... 60

Table 3.3 Equivalent deterministic parameters of stress responses at different locations ........... 62

Table 4.1 Divergence dynamic pressures $\left(q_{\text {div }}\right)$ obtained by different approaches ..................... 87

Table 4.2 Statistics measures of divergence dynamic pressures due to various combinations of

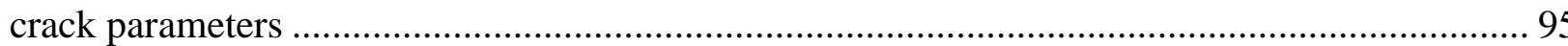




\section{Chapter 1: Introduction}




\section{Introduction}

It is realistic to expect an aircraft having some damage caused by various reasons such fatigue induced cracks, impact with an object during, gust overload during its flight, etc. Nguyen et al. [1] have identified several such damage. Such damage can adversely affect the flight performance of the aircraft. Thus, in 2007, NASA launched a research initiative called Integrated Resilient Aircraft Control (IRAC) to study the effects of damage with the ultimate objective of assessing and increasing the survivability of the aircraft under various adverse conditions. This identified three research sub-topics for the development of computationally efficient: (i) methods for calculating the load constraints that are useful for control adaptation given damage, (ii) next generation methods for estimating the progression of the damage and (iii) a probabilistic methodology to estimate damage growth and remaining life considering all significant uncertainties of the event and the system. The focus of these sub-topics was on the effect of the so-called discrete source damage. Discrete source damage, while not leading to an immediate loss of the flight vehicle, may induce substantial reduction in the strength and stiffness that could greatly reduce the load carrying capability of the flight vehicle structure [2-4]. A rapid assessment of the damage and its effect on the further load carrying capability (maneuver loads) of the aircraft during the remainder of the flight is, thus, of crucial importance.

\subsection{Research Focus of the Study}

Discrete source damage is of special concern if it occurs in the aircraft wings as the wings are among the most important structural and aerodynamic components of any aircraft. The damageinduced reduction of the wing stiffness may require unexpected changes in control system of aircraft [2] as it may result in altered aeroelastic forces and wing deformations. Such damage may also grow with the time during the flight, further worsening the safety of the aircraft. It is thus important to be able to calculate the altered wing response in the presence of damage as well as to examine the growth of damage with time to determine if the aircraft can land safely before the damage becomes too large. The research in this study addresses these three objectives of calculating (a) the response of a damaged wing under static and dynamic loads that are likely to be applied to an aircraft wing, (b) the growth of a crack with time under applied loads and (c) the changes in divergence condition initiated by damage. Since there can be uncertainties in the 
crack location and size as well as in the applied loads, this study considers the effect of these uncertainties in the wing response and crack growth.

The aircraft wings can be in different shapes and have different geometric and structural characteristics. To accurately represent the structural characteristics of such wings for a numerical study, one would need a finite element model of the beam. However, to formulate an analytical/numerical methodology to investigate the two aforementioned aims with reduced computational demand, herein in this study a simple shape of a thin walled beam of closed cross section is assumed to represent a wing. The damage in this beam is represented by the presence of a crack, the size and location of which are assumed to be uncertain quantities represented by random variables. Such a simplified model can be analyzed for different loading and damage scenario relatively easily without attempting to use a large finite element model with several thousand degrees of freedom. This study with a simple representation of a wing and damage is expected to provide the initial guidance about including the effects of the load and damage uncertainties in more realistic performance analysis and evaluation of actual aircraft wings.

The research framework of this study is focused around three related topics dealing with effects of crack on the response and performance of wings represented by thin-walled beams. In the first part, the focus is on the global response of a thin walled beam in the presence of a crack. This provides a basis to examine the effects of the random crack parameters on the global response quantities such as the angle of twist and deflections of the beam. Crack parameters that are considered are the size of the crack and its location along the span. The impact of the crack is evaluated in probabilistic terms such as the response exceeding a threshold. Although it is not a part of this research, this methodology can also be extended to examine the effect of the uncertainties on the control system constraints.

The focus of the second part of the study is on the local analysis where the growth of a crack in a thin walled beam subjected to external stochastic loads is of major concern. In this part also a cantilevered thin-walled beam of rectangular cross section with a crack is used as a simple representation of a damaged wing. Like the first part, the initial crack length and crack location are assumed to be random variables. 
The third part of study focuses on the effect of the crack on the aero-elastic response of the wing, again represented by a thin-walled beam. The interaction of the aero-dynamic loading with the twisting response of the wing can lead to a phenomenon, called divergence. Divergence in general can be estimated without difficulties; however, when damage is present, this condition is subjected to change. This study focuses on the effect of the crack characteristics such as its size and location on the divergence dynamic pressure. For random characteristics of the crack, the probability of the divergence dynamic pressure falling below a limit state value is also evaluated using the safety index approach.

Inclusion of damage and examining its impact on the performance of a structure like an aircraft wing accurately under dynamic and static loads is a difficult problem. Even for a wing modeled as a thin-walled beam, examining the effect of a damage like a crack which introduces discontinuity in the structural model requires special approaches in modeling to capture the damage effect accurately in calculating the response and evaluating its impact on the structural performance. To accurately include the effect of a crack in static and dynamic situations and to implement the probabilistic concepts in the analysis of the assumed structures, a handful of discontinuity modeling approaches and probabilistic approaches were reviewed in this study in order to select the most appropriate approach for the problem at hand. In the following, we present a survey of this literature review to provide the background to a reader about approaches that are used in the following chapters of this study.

\subsubsection{Modeling of Thin-walled Beams}

Modeling of torsion and thin-walled beams has been of interest for more than a century and several theories have been developed. At the early stage of development, bending and torsion in thin-walled beam had been considered independently following the treatment for a beam of solid cross-sections. Theories on bending are well established and they thus are discussed here only for the completeness sake. The Euler-Bernoulli beam theory which ignores shear deformations and Timoshenko's beam theory which includes shear deformation have been used to describe the bending of solid cross as well as section thin-walled beams. Development of theories for thinwalled beam under torsion has many interesting aspects due to warping that are worth discussing. 
In 1853, St.Venant [3, 4] was perhaps the first one to address the issue of torsion in beams without warping. Prandtl [3, 5] later introduced the membrane analogy approach in 1903, making it possible to apply St.Venant's theory to thin-walled cross-section. In 1905, Timoshenko introduced the concept of non-uniform torsion with warping when the governing equations of an I-beam with one end fixed were derived [6]. In 1921, Maillart [3] introduced the concept of shear center and explained the coupling between torsion and bending in unsymmetrical cross-sections.

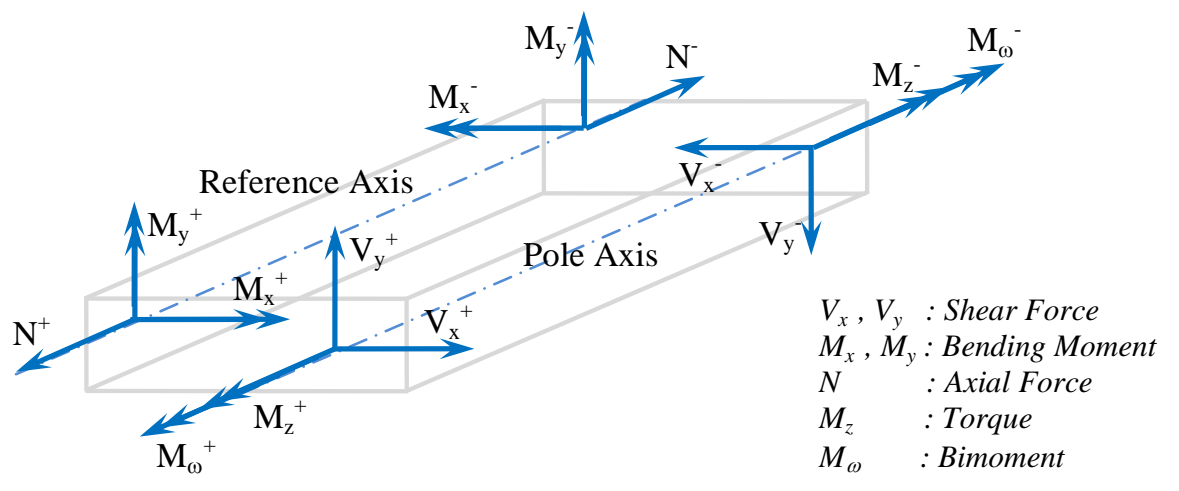

Figure 1.1 Free body diagram of a flexural-torsional member

During 1930s, Vlasov [7] published a book in Russian describing a generalized theory of coupled flexural-torsional of thin-walled beams currently recognized as Vlasov's beam-torsion theory. His theory included an additional component subjected to the restraining warping and this is currently referred as "Vlasov shear". Timoshenko and Goodier [7] also developed independently a generalized theory for coupled flexural-torsional beams [5]. In 1954, Benscoter $[4,8]$ introduced a more generalized theory for thin-walled beams with closed cross-section assuming an additional warping degree of freedom. This warping degree of freedom was assumed independent of the rate of twist, thus allowing the term of shear strain to be described in an exact sense. Since then, the theories by Vlasov and Benscoter have been used as the basis in most studies of thin-walled beams.

Some important contributions that are worth acknowledging were made during 1960 and 1980. In 1964, Capurso [11] presented an idea of generalized higher order warping functions by incorporating additional degrees of freedom to better define the deformation modes of a crosssection. Nishino et al. [9] in 1976 introduced a shearable theory for closed cross section thin- 
walled beams that took into account the transverse shear effect and distortion of cross-section. In 1977, Bishop and Price [10] published a work, demonstrating how to include the effects of shear deformation and rotary inertia in flexural-torsional beam vibration regardless of the warping stiffness. They suggested that these effects are negligible in the deformation shapes of fundamental modes but become appreciable in the corresponding natural frequencies. In 1992, Capuani and Laudiero [11] generalized Timoshenko's beam formulation to use in coupled vibration analysis by including the effect of shear strains due to non-uniform bending and torsion. The thin-walled beam torsion and bending theories proposed so far can be categorized into four groups $[12,13]$.

Euler-Bernoulli's theory: This beam theory for bending of Euler-Bernoulli was extended to include the coupled flexural-torsional effects by combining with St.Venant's shear as shown in Dokumaci $[14,15]$ or St.Venant's and Vlasov's shear as in Bishop [16].

Vlasov's theory: Vlasov originally introduced a thin-walled beam theory for non-uniform torsion considering both St.Venant's and Vlasov's shear components. The theory basically generalized Euler-Bernoulli's theory by taking the effect of rotary inertia into consideration [12]. This theory is discussed in many textbooks on thin-walled beam [4, 5, 17]. Applications based on this theory can be found in [17-22].

Timoshenko's theory: This theory takes into account the rotary inertia and the shear deformation which allows the cross-section to deform in more realistic fashion. Early works based on this theory did not include the effect of restraining warping such as in [10] while later works that include warping stiffness can be found in [12, 13, 18-23].

Benscoter's theory: The assumption of warping displacement being dependent on the rate of twist could lead to significant errors in calculating shear stresses near the location of warping restraints and in closed cross-sections. Benscoter [8], thus, introduced a more generalized approach of employing an additional warping degree of freedom to describe displacement fields independently. This theory is illustrated in [13, 17-19]. 


\subsubsection{Thin-walled Beam of Discontinuous Cross Sections}

In many situations, the thin-walled beams may have discontinuities in their cross sections introduced by cuts and openings. For the analysis of such a structure with sharp variation at interfaces of open and closed cross sections, classical thin-walled beam theories mentioned above cannot be applied without any modifications [17]. The sharp variations introduce discontinuities requiring special compatibility considerations. In classical beam theories, this issue had not been discussed, until Gunnlaugsson and Pedersen [18] in 1982 introduced the concept of least-square compatibility conditions. The idea proposed a practical way to minimize differences of warping displacements across interfaces between two contiguous beams possess different cross-sections. The conditions of least squares were implemented into Benscoter's beam model including transverse shear deformation. Tralli [19] in 1986 presented the use of a hybrid beam-plate element to solve the same problem of a beam subjected to discontinuities or sharp variations at interfaces. In 1991, Pedersen [20] further improved this earlier work with Gunnlaugsson [18]. This work utilized the concept of generalized higher-order warping functions originally introduced by Capurso [21] to provide more accurate results both for static and dynamic responses [22]. Prokic [17, 23, 24] published a new warping function to be used in three-dimensional thin-walled beams. The approach is able to handle a beam of any arbitrary closed and open cross-sections as well as the issue of discontinuity. In 1996, Park et al. [25, 26] used the work of Gunnlaugsson and Pederson [18] in their study on the effect of shear lag on deflection and stress concentration caused by shear warping deformation.

Shakourzadeh et al. [13] published a work discussing on differences between Vlasov's and Benscoter's beam theory. Their results showed some pitfalls of Vlasov's beam when applied to beams of closed-sections. Due to the complexity of full three-dimensional analysis of Prokic's works, Saade et al. [27] published a work that simplified Prokic's works by employing a single warping function instead of three-dimensional warping functions. This work gains the advantages from two different theories by utilizing Vlasov's beam in open cross-section for simplicity whereas applying Benscoter's beam in closed cross-section requiring the shear flow along periphery. 


\subsubsection{Vibration of Thin-walled Beam}

This section is dedicated to the discussion on the solutions of thin-walled beam vibration problem. The review concentrates only on the solutions to torsional and coupled flexuraltorsional vibrations as the pure flexural and axial vibrations are not the cases of interest in this study. Different solution approaches have been developed independently. Exact analytical solutions are feasibly only for beams with simple boundary conditions [28] while approximate solutions are available through many techniques of discretization such as Lumped mass, Rayleigh-Ritz, Assumed mode, Galerkin and Finite element methods. Basically, these approaches can be divided into two categories. The first discretizes the continuous system by lumping the masses at discrete points while the second discretizes by assuming the final form of solution as the product of time and space-dependent functions [29]. All publications demonstrating applications of these methods to thin-walled beams are briefly discussed chronologically.

In 1954, Gere [30] published the classical work on exact solutions of pure torsional vibration problems of thin-walled beams of doubly symmetric cross-sections with various boundary conditions. Gere and Lin [31] published another paper on both exact and approximate solutions of coupled flexural-torsional vibrations for beams of non-symmetric cross-sections. In 1973, Falco and Gasparetto [15] applied the method of transfer matrix to a mass-discretized system so as to determine the natural frequencies and corresponding modes for the coupled flexuraltorsional vibration problem excluding warping rigidity.

Hallauer [32] in 1982 derived analytically the expression of dynamic stiffness matrix for coupled flexural-torsional Euler's beams. In 1984, Rozmarynowski and Szymczak [33] derived a solution to the nonlinear problem of torsional vibration of bi-symmetric cross-section using a finite element method. Noor et al. [34] developed a mixed finite element method to solve the coupled flexural-torsional vibration of a curved beam. In 1990, Ahmad and Guile [35] presented a work on the comparison of coupled flexural-torsional natural frequencies between beam and shell models based on finite element. Results show consistency between models at fundamental modes. Banerjee and Williams [36] in 1992 demonstrate an analytical method to obtain the exact expression of dynamic stiffness matrix for coupled flexural-torsional Timoshenko's beam. In 
1993, Mcgee et al. [37] presented an analytical solution to the case of torsional vibration under a pre-twisted loading.

Bercin and Tanaka [12] in 1997 employed the method proposed by Banerjee and Williams [36] to obtain numerical results for the case of a thin-wall beam of monosymmetric crosssection. They found that the effects of warping stiffness, shear deformation and rotary inertia were significant for the modal frequency when the thickness of cross-section increases. Eisenberger [38] derived an exact solution of modal frequencies and dynamic stiffness matrix for torsional vibration of variable cross-section. In 1999, Wu [39] proposed an alternative method to estimate natural frequencies and mode shapes. The approach consisted of applying an analyticnumerical combined method to the equivalent two degree of freedom spring-mass system.

In 2000, Kuang [40] utilized the Galerkin technique to approximate the frequencies and mode shapes of coupled flexural-torsional vibrations of shear walls. Ambrosini [41] presented the use of state-variable technique to analyze the free vibration of coupled flexural-torsional beams in the frequency domain. Mohri [42] studied the vibration characteristics of buckled thin-walled beams. Prokic [43, 44] published two companion papers in 2005 and 2006 giving comprehensive derivations of coupled flexural-torsional Vlasov's and Timoshenko's beam vibrations including their exact solutions for the case of uniform simply-supported beams. In 2006, Kapania and Kim [45] investigated the uses of various orthogonal polynomial functions in the approximate solutions of flexural-torsional vibrations of slewing beams. They found that the use of simple Legendre polynomials provide the best efficiency in obtaining results compared with other types of orthogonal polynomials. Senjanovic [46] demonstrated the use of one dimensional finite element model to approximate the dynamic response of ship hull. Several other studies by Song and Librescu [47], Librescu [48], Suresh [49], Qin [50], Librescu [51], Jun et al. [52] and Wang [53] are also available in the literature describing the solutions to thin-walled beam vibrations of anisotropic materials.

\subsubsection{Probabilistic Methods and Reliability Analysis}

The concepts of probability were introduced to engineering applications to deal with the issues of uncertainty occurring naturally in the design of engineering structures. The review of 
literatures on this section focuses on the development of these concepts and methodologies as applied to the field of structural engineering. Both static and dynamic methodologies are discussed for the sake of completeness.

\subsubsection{Reliability Method}

The concept of reliability-based design was originally introduced by Freudenthal et al. [54-56] during 1950s and 1960s. Since in most engineering applications, one would usually have information only about the first two moments of the random variables and not full distribution information, in 1967 Cornell [57] introduced the safety index approach as a measure of safety or probability of survival of structures in terms of the first two moments of its load and capacity variables. In 1969 and 1974, Cornell and Ang [58, 59] formalized this concept of reliability evaluation approach called as the first-order reliability method (FORM) or particularly first-order second-moment method (FOSM). The approach used the first-order linear approximation of limit state function. Due to neglecting higher order terms, the result contained significant error for the case of non-linear limit state function and the safety index of reliability could not be uniquely defined for different but equivalent forms of the limit state function. Realizing this, in 1974 Hasofer and Lind [60] introduced the use of transformed or reduced coordinated system to normalized random variables. This idea enabled the use of an optimization technique based on Lagrange multipliers to obtain the invariant safety index values for nonlinear limit state functions. This approach is currently referred as Hasofer-Lind method. In 1976, Rackwitz [61] formulated an iterative algorithm to circumvent the inconvenient procedure of solving multiple equations in the Lagrange multiplier approach. Due to the limitation of Hasofer-Lind method being only applicable to normal variables, Rackwitz and Fiessler [62] in 1976, introduced a method of estimating the equivalent normal distribution of non-normal variables. In 1978, Rackwitz and Fiessler [63] published an alternative algorithm of iteration based on NewtonRaphson recursive formula. This method has an advantage over Rackwitz's first method as it did not require the definition of the limit state equation in closed-form. This procedure is currently recognized as the Rackwitz-Fiessler algorithm. However, the algorithms sometimes do not converge to yield the final result in some certain cases due to numerical divergence. A method proposed by Broyden-Fletcher-Goldfarb-Shannon method or so-called BFGS algorithm is recommended for those particular cases [64]. 
To improve the accuracy of the results obtained using the first-order approximation (FORM), the second-order reliability method (SORM) was first introduced by Fiessler et al. [65] using the technique of quadratic approximations. In the case of correlated random variables, an additional step of orthogonal transformations needs to be incorporated into the formulations. Der Kiureghian and Liu developed a semi-empirical formulation to facilitate the complexity in obtaining the correlation matrix [64]. Most of the time, the solution of a structural engineering problem can only be obtained numerically and its limit state function is not explicitly defined in a closed form. Those standard approaches are inconvenient and complicated to be used to obtain the results. Therefore, computational approaches are preferable for the cases of implicit limit state function. Three categories of approaches are essentially Monte Carlo Simulation, the Response Surface Approach and Sensitivity-based Analysis [64]. Comprehensive details of derivations and calculations are described in several published books in this area by Ang [66], Elishakoff [67], Melchers [68], Rao [69], Haldar [64], Nowak [70], Nikolaidis [71, 72], Choi [73] and Maymon [74].

\subsubsection{Random Vibration of Beam-type Structure}

Probabilistic structural dynamics was originally developed in the late 1950s. Its objective was to deal with the issue of uncertainty in the motion of structures due to unpredictable excitations. Stochastic input yields the output of stochastic characteristics [75]. Books by Crandall [76] in 1959, Crandall and Mark [77] in 1963 and Lin [78] in 1967 are among the classics on this topic, introducing the theory of random vibrations. In 1969, Shinozuka and Yang [79] demonstrated the application of random vibrations to linear structures. In 1980, Davies [80] presented a work on random vibration of beam subjected to impact loads dedicated to the application in piping systems. Elishakoff and Livshits [81, 82] in 1984 and 1989 presented closed-form randomvibration solutions of Euler and Timoshenko beam bending respectively. In 1993, Singh and Abdelnaser [83, 84] derived analytical random-vibration solutions of externally-damped viscoelastic Timoshenko beams and cantilevered composite beams with coupled bending-torsion. Eslimy-Isfahany [85] presented a work in 1996 on the response of an aircraft wing modeled using coupled bending-torsion beam subjected to random loads. Jun et al. [86] in 2004 employed the concepts from a publication of Bercin and Tanaka [12] so as to derive the analytical expressions of the response of a mono-symmetric thin-walled beams subjected to random 
excitations using combined approach of the normal mode superposition and frequency response. Jun and Xiading [87] also extended their derivations to composite thin-walled beams. Fundamental concepts of random vibrations are now given in several books in this area by Nigam [88, 89], Newland [90], Lin and Cai [75], Lutes and Sarkani [91] and Soong and Grigoriu [92].

\subsubsection{Fracture and Fatigue}

Since this study deals with the development of cracks under uncertain dynamic loading, here a brief review of the literature on this topic related to this study is presented. In particular the focus of this discussion will be on the literature involved in the study of growth of a crack with random properties and modeling uncertainties under random dynamic loading. Early contributions to the area of random fatigue were introduced during 1940s and 1950s in a series of publications by Freudenthal, Gumbel and Heller [93-95]. These early studies focused only on the initiation of cracks. Prominent references in this area are the books by Heller [96], Haugen [97], Provan [98], Sobczyk [99] and two latest published books by Schijve [100] and Castillo [101].

A methodology based on theory of fracture mechanics was originally proposed during early 1960s for fatigue crack growth investigations. The approach focused on the continuum regime or intermediate phase of crack propagation between the phases of crack initiation and final fracture phase. The approach assumes that even a crack size below a critical crack level will keep propagating when subjected to cyclic loading [102]. This approach is appropriate for failure analysis of damaged structures in which damage appears as a long crack. The fundamental concept of this approach is that the rate of crack growth is correlated to the change in stress intensity factor, which is described by an empirical differential-equation model. Within the intermediate zone of crack propagation, concepts of linear elastic fracture mechanics are applicable. Three classical models are Paris-Erdogan equation [103], Forman equation [104] and Walker equation [105]. Some slight differences between these models are mainly due to the use of the constants obtained by fitting experimental data. The load ratio or R-ratio is recognized to be an important factor in these fatigue crack growth models $[99,106]$. Two important articles in the area were published in 1979 by Skelton [110] and Wanhill [111]. Skelton [107] proposed a

method to be used for fatigue crack growth subjected to high strain and Wanhill [108] published 
a study of fatigue crack propagation tested on a flight simulation. The failure of aerospace structural element is sometimes governed by low-cycle fatigue. In 1984, Kujawski and Ellyin $[109,110]$ developed a particular crack growth model taking into account of plastic strain energy occurring when overload exists as in case of low-cycle fatigue. For high-stress and low-cycle fatigue, Rahman et al. [111] in 1997 presented three different schemes to calculating J-integral based on EPFM to be used in Paris equation instead of stress intensity factor of LEFM. Comprehensive review of crack propagation models can be found in Beden et al. [112].

The stochastic model of crack propagation was introduced during late 1970s. In 1979, Virkler [113] found from test results that the statistical distribution of cycle to failure could be represented by log normal distribution. Yang and Donath [114] in 1983 and Yang et al. [115] in 1985 published works associated with stochastic crack propagation in fastener holes. Statistical distributions of crack size over time and probability of failure were obtained analytically. Results from their empirical model were consistent to the experimental data for various load spectra. In 1985, Lin and Yang [116] introduced the approximation of time-dependent crack size using a Markov process. Results showed consistency for practical applications as far as fatigue life is not too short. Oh [117] in 1979 and Sobczyk [118] in 1982 proposed an idea of using Markov chain and Markov diffusion process to model the stochastic growth of crack. Sobczyk [122] in 1989 and Trebicki [123] in 1991 considered the intermittent behavior of stochastic crack growth using cumulative jump model based on Poisson process while Ditlevsen and Sobczyk [119] included an additional effect due to retardation into the same model. Liu et al. [120] in 1996 introduced the use of two different first-order reliability methods (FORM) to analyze the stochastic growth of crack. The Lagrange multiplier based FORM approach appeared to be the most efficient and accurate. Other models and comprehensive discussions on probabilistic modeling of crack propagation can now be found in several books such as by Bloom [121], Ellyin and Fakinlede [122], Bogdanoff and Kozin [123], Provan [98], Sobczyk et al. [99], Ellyin [124], Maymon [125, 126] and Castillo [101].

\subsection{Dissertation Organization}

This dissertation consists of three main chapters dealing with the three topics mentioned earlier. Each chapter is written as a technical paper, ready for submission for its possible 
publication. The papers in Chapter 2 and Chapter 3 have already been published in the AIAA SDM conference held in Florida in April 2010. A brief outline of each chapter is provided in the following.

Chapter 2 presents the formulation for the global response analysis of a thin-walled cantilevered beam with a longitudinal crack. The model of rectangular beam with a crack parallel to the beam on one of the faces is considered to represent a damaged wing. The thin-walled beam is modeled using the Benscoter [8] theory coupled with Gunnlaugsson and Pedersen [18] compatibility conditions to deal with the issue of warping and deformation compatibility between the open and closed cross sections. The uncracked portion of the beam is represented by a closed cross section thin-walled beam and the cracked portion by an open cross section thin walled beam. The governing equations of the beam for general loading configuration with flexural, axial and torsional loads are obtained by the principle of virtual work. The couple equations of motion are solved analytically in closed-form using a special form of eigenfunction expansion approach so-called the Jordan canonical form to define the beam response under the applied loads. This closed form solution is then used in the probabilistic analysis where the parameters of crack location and crack length are considered as random variables with known mean and standard deviation values. The probability of a response quantity exceeding a limiting value is obtained using the safety index approach. Numerical results are obtained for the probability of the cracked beam response exceeding a factor time the un-cracked beam response using the safety index approach. The numerical results obtained in the first version of this study were presented in a paper in the SDM conference in April 2010 [127].

Chapter 3 describes a local analysis approach wherein a methodology for determining the growth of a crack under random dynamic loading is presented. The crack growth in the study is defined by the Forman equation which is a modified version of the famous Paris-Erdogan crack growth model. This model requires the definition of the stress field in uncracked beam, stress intensity factor, stress factor ratio and information about stress cycles. To obtain the stress cycle information for the beam, the beam is again modeled by Benscoter [8] thin-walled theory with combined bending torsion effects. The equations of motion of such a beam subjected to generalized loading are obtained by the variational methods. These equations of motion can be 
solved analytically using the state-space form approach or by the Rayleigh Ritz approach with assumed modes. Here in this study, the results are obtained by the latter approach. The loading on the beam is defined by gust spectra characterized by a spectral density function. The equations of motion are solved to obtain the stress response spectral density function. This spectral density function is used to calculate the spectral moments of the stress response that are needed to define the probability density function of the stress peaks. The probability density function of the stress peaks is used to define the statistics of the peak stress response such as equivalent stress cycles and stress ratio factors that are needed in the crack growth analysis. Since the crack parameters, the location and the size, along with a few other parameters in the crack growth model equation are assumed to be random variables, the crack growth must also be defined in probabilistic terms. That is, at a given time the size of the crack is a random variable and thus must be characterized by its probability density function. To determine this probability density function of the crack length, Monte Carlo simulation is used. For each simulated set of variable values, the growth model equation is solved numerically to define the time evolution of the crack size. At a given time, the sample values of the crack size obtained for each simulation define the sample probability distribution of the crack length. The probabilistic comparison of the crack length with the fracture toughness provides a good estimate of the probability of failure with time. The results of the initial study completed on this topic are presented in a SDM Conference paper [128]. This study is hoped to provide the guidelines for the estimation of the time to failure and flight risk posed by the presence of a crack on the wing of an aircraft.

In Chapter 4, the analytical solution developed in Chapter 2 is extended for aero-elastic analysis of the beam representing the wing. In particular, the closed form analytical solution of a beam with a crack is used to calculate the divergence dynamic pressure for the wing considering the interaction between the angle of attack and the twisting moment acting on the wing. Two approaches herein called as the iterative and direct approaches are used. The impact of the uncertainties in the crack parameters (its size and location along the beam) on the divergence dynamic pressure is also investigated probabilistically.

The dissertation ends with Chapter 5 which finally presents an overall summary of the study and its main concluding remarks. 


\section{Chapter 2: Probabilistic Analysis of a Thin- walled Beam with a Crack}


Probabilistic Analysis of a Thin-walled Beam with a Crack* Chalitphan Kunaporn, Mahendra P. Singh, Mayuresh J. Patil and Rakesh K. Kapania Virginia Polytechnic Institute and State University, Blacksburg, VA 24061

This paper presents the analysis of a thin walled beam with a longitudinal crack of random location and size. The research objective is to understand the response characteristics of such a damaged beam with the ultimate goal of examining the growth of a crack under random loading. This initial study is expected to guide the future analysis of an aircraft wing with uncertain damage characteristics. An analytical method is presented to obtain the response of a simple thin-walled beam of a closed cross section with a longitudinal crack of finite size. For random location and size of the crack, the methodology for the first order reliability analysis with analytically calculated response is described. The numerical results of the reliability analysis of the beam for the reliability defined as the non-exceedance of a limit state are presented.

\subsection{Introduction}

The damage in a wing can significantly affect the performance of an aircraft. With the motivation of examining the performance of an aircraft with a damaged wing, this study examines the response of a hollow thin-walled beam with a longitudinal crack. The crack location and size are considered random. First the paper presents an analytical formulation to obtain the response of the beam as a function of the crack parameters followed by probabilistic and reliability analysis.

The currently available thin-walled beam theories are adequate to model beams without a crack and are able to provide accurate values of the overall response. They require appropriate modifications to include a crack and such modification are described in this study. However, even with such a modification, they are not able to accurately capture stresses near the crack. So the present work focuses primarily on overall deformation analysis of such a beam.

\footnotetext{
* This article is prepared for submission to the AIAA journal.
} 
A beam with a longitudinal crack can be represented by three interconnected thin-walled beams. The portion of the beam with the crack can be modeled as an open section thin-walled beam, whereas the other two sections as closed section thin-walled beams. Such a beam when subjected to lateral loads or a twisting moment is expected to warp. The thin-walled beam theories that allow us to include warping can be mainly divided into two categories: Vlasov beam theory $[4,5,22,43,129]$ and Benscoter beam theory $[8,13,18,20,26,130,131]$. In this study, the derivation is based on Benscoter beam theory where the warping degree of freedom is considered independent of the rate of twist. The equilibrium equations and the boundary conditions for the system are derived using the principle of virtual work. The coupled equilibrium equations are then expressed in state space form which allows us to solve them using the Jordan canonical form approach. The accuracy of the calculated response by the proposed approach is validated by a detailed shell finite element analysis.

To investigate the effect of random characteristics of a crack on the performance, a first order reliability analysis method [61-64, 66] is used. The higher order analyses [65] could also be used to improve the reliability estimates especially if the limit state boundary is highly nonlinear. This is, however, not attempted in this study as the limit state boundaries used in this study are nearly linear. For the reliability analysis, the limit state is defined in terms of the angle of twist exceeding some limiting value. Since the response quantity of interest is not explicitly defined in closed-form in terms of the crack parameters, the gradients needed for the reliability analysis must be calculated by chain rule, applied at successive analytical steps that are used in the response calculations. The numerical results demonstrating the methodology are presented.

\subsection{Analytical Model of a Thin-walled Beam with a Crack}

The damaged structure is modeled as a hollow cantilever thin-walled beam of a rectangular shape shown in Figure 2.1. The damage is represented by a longitudinal crack of a given length on the top face. The beam is divided into three parts with the cracked part between the two undamaged parts. The undamaged beam portions are represented as the closed cross-section beam and the damaged portion as the open cross-section beam as shown in Figure 2.1. The kinematics of thin-walled bar is described using the combination of the Cartesian $(x, y, z)$ and 
orthogonal curvilinear $(s, n, z)$ coordinates. For convenience, the global $(Z)$ and local $\left(z_{i}\right)$ coordinates are used interchangeably.

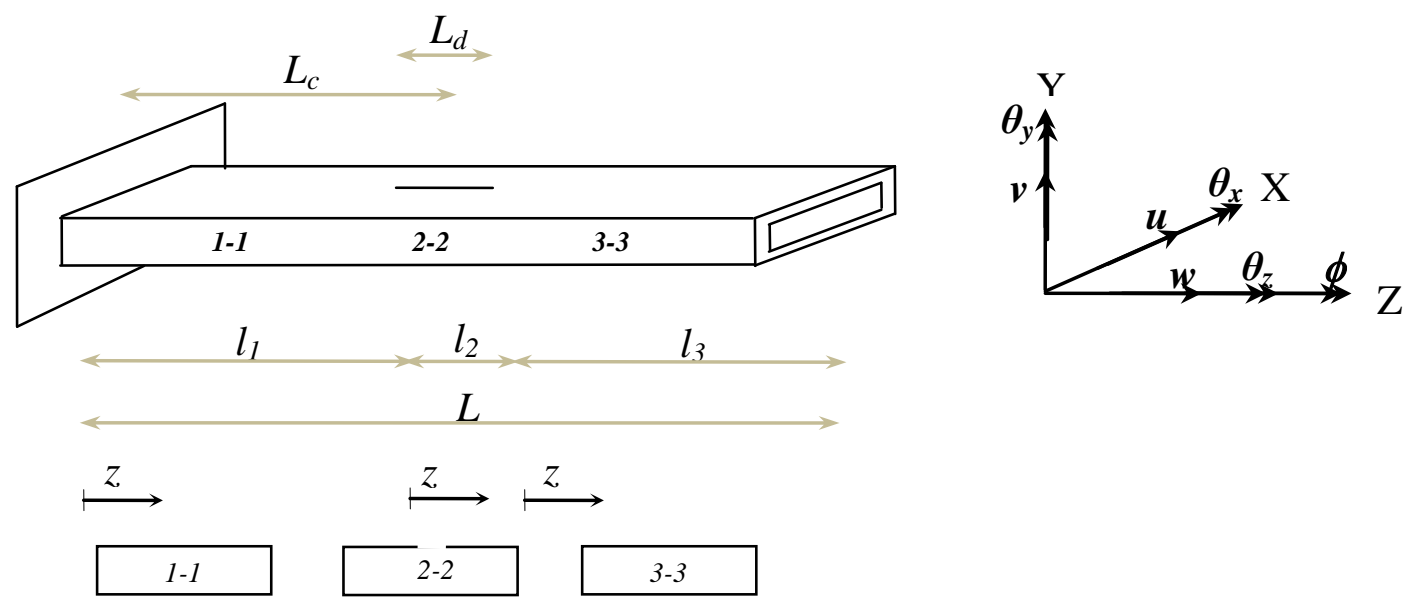

Figure 2.1 Damaged structure modeled with a cracked thin-walled cantilever

The closed cross-section is assumed to be not deformable in its own plane. Therefore, only rigid body motions are allowed for the in-plane displacements. The warping in both cross sections introduces axial deformations along the $z$-direction and shear deformations in the plane of the cross section. An arbitrary point, $P\left(x_{p}, y_{p}\right)$, or so-called "Pole" is used as the reference to describe all displacement fields. The pole of a cross-section along the beam span is chosen at the shear centers and the angle of twist, $\theta_{z}$, is assumed to be about this point. Only two coordinates, $\theta_{z}$, and warping displacement, $w$, are needed to define the displacement and stress field to derive the governing equations. The tangential displacements, $v_{s}$, and the out-of-plane warping displacement, $w$ are used to define the displacement field in the $(s, n, z)$ coordinate system. To calculate the warping function of a thin-walled section, we choose a reference point, $C O\left(x_{c o}, y_{c o}\right)$, where the coordinate $s=0$ for contour integration. The dimensions $b$ and $h$, respectively, are the width and height of the beam cross-section. 


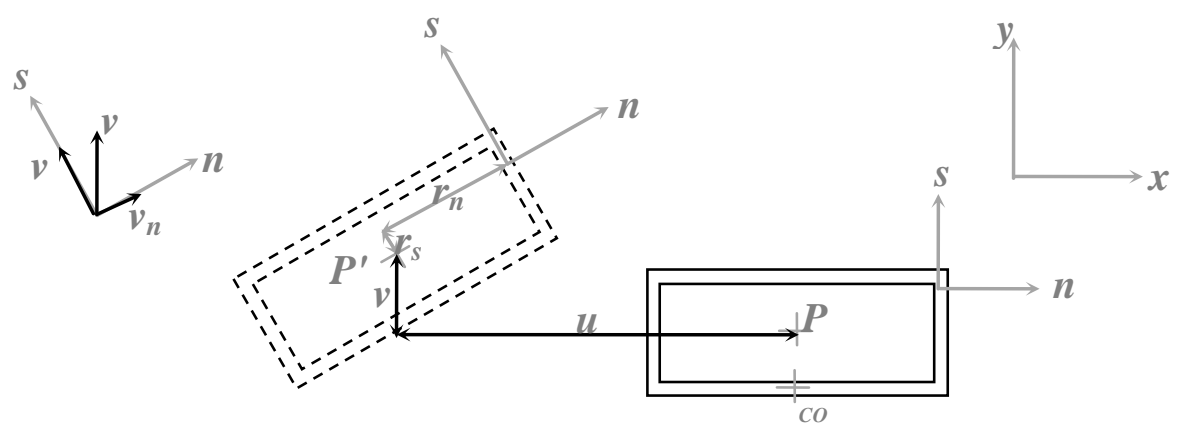

Figure 2.2 Kinematics of a beam cross-section

An additional degree of freedom, $\phi(z)$, is introduced as the measure of warping. As in the Benscoter's thin-walled beam theory [8], it is assumed to be independent of the axial rotation degree of freedom of the beam. In terms of these, the tangential and warping displacements can now be written as follows [18]:

$$
\begin{gathered}
v_{s}(s, n, z)=u(z) \frac{\partial x(s)}{\partial s}+v(z) \frac{\partial y(s)}{\partial s}+r_{n}(s, n) \theta_{z}(z) \\
w(s, n, z)=w_{o}(z)+y(s) \theta_{x}(z)-x(s) \theta_{y}(z)-\psi(s, n) \phi(z)
\end{gathered}
$$

where $u(z)$ and $v(z)$ are the displacements of the pole in $x$ and $y$ directions, respectively; $w_{o}(z)$ is the warping displacement of the contour origin; $\theta_{x}(z)$ and $\theta_{y}(z)$ are the rotations of pole with respect to the $x$ and $y$ axis, respectively; $\theta_{z}(z)$ is the angle of twist; $\psi(s, n)$ is the warping function; $\phi(z)$ is the measure of warping that in special cases can be approximated to the rate of twist; $\theta_{z}^{\prime}$. ( ) denotes the derivative with respect to $z ; x(s)$ and $y(s)$ are Cartesian coordinate rewritten in terms of the tangential coordinate. In the linear theory, the rotations of pole are described as follows:

$$
\begin{aligned}
& \theta_{x}(z)=\gamma_{y z}(z)-v^{\prime}(z) \\
& \theta_{y}(z)=\gamma_{x z}(z)-u^{\prime}(z)
\end{aligned}
$$

where $\gamma_{x z}$ and $\gamma_{y z}$ are transverse strains. 
In the shear deformable beam theory, the Euler-Bernoulli hypothesis is no longer applicable as $\gamma_{x z} \neq 0$ and $\gamma_{y z} \neq 0$ and the transverse strains are included in the derivation. Infinitesimal strain tensor in linear theory is expressed in terms of displacements. Based on the thin-walled theory, the energy equation requires only two main strain components, $\varepsilon_{z z}$ which is the normal strain in $z$ direction and $\gamma_{s z}=2 \varepsilon_{s z}$ which is the shear strain in $s z$ plane. They are defined as follows:

$$
\begin{gathered}
\varepsilon_{z z}=\frac{\partial w}{\partial z} \\
\gamma_{s z}=2 \varepsilon_{s z}=\frac{\partial v_{s}}{\partial z}+\frac{\partial w}{\partial s}
\end{gathered}
$$

Both the open and the two closed cross-sections have the same geometric center but different shear centers, thus, causing the centers of twist to vary along the beam length. Although the beam is subjected only to torsion, it is no longer the case of pure torsion, but the coupled bending and torsion must be considered. The governing equations are derived using the principle of virtual work. The following derivation is based on isotropic materials; however, it could be generalized to use for the composite materials as shown in [22]. In static analysis, the principle of virtual work can be written as follows:

$$
\begin{gathered}
\delta W_{\text {Internal Work }}-\delta W_{\text {External Work }}=0 \\
\iiint_{V} \sigma \delta \varepsilon d V-\left(\iiint_{V} B \delta u d V+\iint_{S} T \delta u d S\right)=0
\end{gathered}
$$

where $B$ and $T$ stand for the body forces and the tractions, respectively.

For a thin-walled beam cross-section, the body forces are zero in a static case and the term of work due to strain energy can be simplified. Considering the tractions in the form of distributed loads along the span and concentrated loads at certain locations of the span, the principle of virtual work can be written as follows:

$$
\begin{aligned}
& \int_{a}^{b}\left[\int_{A}\left(E \varepsilon_{z z} \delta \varepsilon_{z z}+G \gamma_{s z} \delta \gamma_{s z}\right) d A\right. \\
& \left.-\left(p_{x} \delta u+p_{y} \delta v+p_{z} \delta w+m_{x} \delta \theta_{y}+m_{y} \delta \theta_{x}+m_{z} \delta \theta_{z}-m_{\omega} \delta \phi\right)\right] d Z \\
& -\left(P_{x} \delta u+P_{y} \delta v+P_{z} \delta w+M_{x} \delta \theta_{x}+M_{y} \delta \theta_{y}+M_{z} \delta \theta_{z}-M_{\omega} \delta \phi\right)=0
\end{aligned}
$$


where $E$ is the Young's modulus and $G$ is the modulus of torsional rigidity; $p_{x}, p_{y}$ and $p_{z}$ are the distributed forces in $x$-, $y$ - and $z$-direction, respectively; $m_{x}, m_{y}$ and $m_{z}$ are the distributed moments with respect to $x, y$ and $z$ axis, respectively; $m_{\omega}$ is the distributed warping torque about $z$ axis; $P_{x}$ and $P_{y}$ are the shear forces in $x$ and $y$ directions, respectively; $P_{z}$ is the axial force; $M_{x}$ and $M_{y}$ are the bending moments with respect to $x$ and $y$ axis, respectively; $M_{z}$ is the torque; and $M_{\omega}$ is the warping moment or the so-called "Bimoment".

In the span-wise direction, the beam model consists of three portions. For convenience, the local coordinates are mainly used as the references in the span-wise direction. The first portion has boundaries from $z_{1}=0$ to $z_{1}=l_{1}$, the second portion with the crack spans from $z_{2}=0$ to $z_{2}=l_{2}$ and the last portion spans from $z_{3}=0$ to $z_{3}=l_{3}$. For the plane of cross-section, however, the global coordinates are still used. The contour origin is chosen so that the warping displacement of the contour origin, $w_{o}(z)$, is zero. Substituting all stress and strain components and applying the principle of virtual work, the system of equilibrium equations as well as the corresponding boundary-continuity conditions are obtained as follows:

$$
\begin{gathered}
-E A w^{\prime \prime}=p_{z} \\
-G J_{\theta y} \theta_{z}^{\prime \prime}+G Q_{y z} \phi^{\prime}-G Q_{x y}\left(v^{\prime \prime}+\theta_{x}^{\prime}\right)-G Q_{y y}\left(u^{\prime \prime}-\theta_{y}^{\prime}\right)=p_{x} \\
-G J_{\theta x} \theta_{z}^{\prime \prime}+G Q_{x z} \phi^{\prime}-G Q_{x x}\left(v^{\prime \prime}+\theta_{x}^{\prime}\right)-G Q_{x y}\left(u^{\prime \prime}-\theta_{y}^{\prime}\right)=p_{y} \\
E \Gamma_{z x} \phi^{\prime \prime}-E I_{x x} \theta_{x}^{\prime \prime}+E I_{x y} \theta_{y}^{\prime \prime}+G J_{\theta x} \theta_{z}^{\prime}-G Q_{x z} \phi+G Q_{x x}\left(v^{\prime}+\theta_{x}\right)+G Q_{x y}\left(u^{\prime}-\theta_{y}\right)=m_{x} \\
-E \Gamma_{z y} \phi^{\prime \prime}+E I_{x y} \theta_{x}^{\prime \prime}-E I_{y y} \theta_{y}^{\prime \prime}-G J_{\theta y} \theta_{z}^{\prime}+G Q_{y z} \phi-G Q_{x y}\left(v^{\prime}+\theta_{x}\right)-G Q_{y y}\left(u^{\prime}-\theta_{y}\right)=m_{y} \\
-G J_{\theta \theta} \theta_{z}^{\prime \prime}+G J_{\theta z} \phi^{\prime}-G J_{\theta x}\left(v^{\prime \prime}+\theta_{x}^{\prime}\right)-G J_{\theta y}\left(u^{\prime \prime}-\theta_{y}^{\prime}\right)=m_{z} \\
-E \Gamma_{z z} \phi^{\prime \prime}+E \Gamma_{z x} \theta_{x}^{\prime \prime}-E \Gamma_{z y} \theta_{y}^{\prime \prime}-G J_{\theta z} \theta_{z}^{\prime}+G J_{z z} \phi-G Q_{x z}\left(v^{\prime}+\theta_{x}\right)-G Q_{y z}\left(u^{\prime}-\theta_{y}\right)=-m_{\omega}
\end{gathered}
$$

The geometric problem parameters used in the above equations are defined as follows: $A=\int_{A} d A, S_{x x}=\int_{A} y d A, S_{y y}=\int_{A} x d A, S_{z z}=\int_{A} \psi d A$ 


$$
\begin{aligned}
& \Gamma_{z z}=\int_{A} \psi^{2} d A, \Gamma_{z x}=\int_{A} y \psi d A, \Gamma_{z y}=\int_{A} x \psi d A \\
& I_{x x}=\int_{A} y^{2} d A, I_{x y}=\int_{A} x y d A, I_{y y}=\int_{A} x^{2} d A \\
& J_{\theta \theta}=\int_{A} R_{n}^{2} d A, J_{\theta z}=\int_{A} R_{n} \frac{\partial \psi}{\partial s} d A, J_{z z}=\int_{A}\left(\frac{\partial \psi}{\partial s}\right)^{2} d A, J_{\theta x}=\int_{A} R_{n} \frac{\partial y}{\partial s} d A, J_{\theta y}=\int_{A} R_{n} \frac{\partial x}{\partial s} d A \\
& Q_{x x}=\int_{A}\left(\frac{\partial y}{\partial s}\right)^{2} d A, Q_{x y}=\int_{A} \frac{\partial x}{\partial s} \frac{\partial y}{\partial s} d A, Q_{y y}=\int_{A}\left(\frac{\partial x}{\partial s}\right)^{2} d A, Q_{x z}=\int_{A} \frac{\partial y}{\partial s} \frac{\partial \psi}{\partial s} d A, Q_{y z}=\int_{A} \frac{\partial x}{\partial s} \frac{\partial \psi}{\partial s} d A
\end{aligned}
$$

The corresponding boundary conditions at the two ends are obtained as follows:

At the fixed end,

$$
\begin{gathered}
u=0, v=0, w=0 \\
\theta_{x}=0, \theta_{y}=0, \theta_{z}=0 \\
\phi=0
\end{gathered}
$$

At the free end,

$$
\begin{gathered}
G J_{\theta y} \theta_{z}^{\prime}-G Q_{y z} \phi+G Q_{x y}\left(v^{\prime}+\theta_{x}\right)+G Q_{y y}\left(u^{\prime}-\theta_{y}\right)=P_{x} \\
G J_{\theta x} \theta_{z}^{\prime}-G Q_{x z} \phi+G Q_{x x}\left(v^{\prime}+\theta_{x}\right)+G Q_{x y}\left(u^{\prime}-\theta_{y}\right)=P_{y} \\
E A w^{\prime}=P_{z} \\
-E \Gamma_{z x} \phi^{\prime}+E I_{x x} \theta_{x}^{\prime}-E I_{x y} \theta_{y}^{\prime}=M_{x} \\
E \Gamma_{z y} \phi^{\prime}+E I_{x y} \theta_{x}^{\prime}-E I_{y y} \theta_{y}^{\prime}=M_{y} \\
G J_{\theta \theta} \theta_{z}^{\prime}-G J_{\theta z} \phi+G J_{\theta x}\left(v^{\prime}+\theta_{x}\right)+G J_{\theta y}\left(u^{\prime}-\theta_{y}\right)=M_{z} \\
E \Gamma_{z z} \phi^{\prime}-E \Gamma_{z x} \theta_{x}^{\prime}-E \Gamma_{z y} \theta_{y}^{\prime}=-M_{\omega}
\end{gathered}
$$

At the interface of the uncracked and cracked portions of the beam, the boundary conditions are defined in terms of the continuity conditions as described in the following.

\subsubsection{Continuity Conditions}

Due to different warping functions used between the closed and the open cross-sections, the discontinuity of warping displacements at the interface of the two sections across the beam portion is inevitable. In order to deal with this issue, the thin-walled beam theory is modified by incorporating the technique proposed by Gunnlaugsson and Pedersen $[18,130]$ to account for the 
compatibility conditions in the standard governing equations. To facilitate the minimization of the difference, they proposed to include additional constants in the representation of the warping displacement only on one side of the interface, as explained in the following.

At the interface of the two different cross-sections, the warping displacement field of the element on the right-hand side is rewritten to include the assumed compatibility constants as follows:

$w^{\text {right }}(s, n, z)=w_{o}(z)+y(s) \theta_{x}(z)-x(s) \theta_{y}(z)-\left[d_{1}+d_{2} y(s)-d_{3} x(s)+d_{4} \psi^{\text {right }}(s, n)\right] \phi(z)$

whereas the warping displacement of the right element remains unchanged as follows:

$$
w^{l e f t}(s, n, z)=\bar{w}(z)+y(s) \theta_{x}(z)+x(s) \theta_{y}(z)-\psi^{l e f t}(s, n) \phi(z)
$$

To calculate the constants and define the compatibility condition, we minimize the following integral norm of the point-wise difference between the warping displacements:

$$
I=\int_{A}\left(w^{l e f t}-w^{r i g h t}\right)^{2} d A
$$

where the superscripts "left" and "right" denote the closed and open cross sections, respectively.

The necessary conditions for the minimization of this functional provide the compatibility conditions and the following four equations to calculate the constant $d_{i}$ :

$$
\begin{array}{lcl}
\frac{\partial I}{\partial d_{1}}=0: & \int_{A}\left(w^{\text {left }}-w^{\text {right }}\right) d A=0 \\
\frac{\partial I}{\partial d_{2}}=0: & \int_{A}\left(w^{\text {left }}-w^{\text {right }}\right) y d A=0 \\
\frac{\partial I}{\partial d_{3}}=0: & \int_{A}\left(w^{\text {left }}-w^{\text {right }}\right) x d A=0 \\
\frac{\partial I}{\partial d_{4}}=0: & \int_{A}\left(w^{\text {left }}-w^{\text {right }}\right) \psi^{\text {right }} d A=0
\end{array}
$$


On expansion, the above equations lead to the following four simultaneous equations for calculating the constants $d_{i}$ expressed in terms of the integration constants of the beam cross sections.

$$
\begin{aligned}
& d_{1} A^{\text {right }}+d_{2} S_{x x}^{\text {right }}-d_{3} S_{y y}^{\text {right }}+d_{4} S_{z z}^{\text {right }}=S_{z z}^{\text {left }} \\
& d_{1} S_{x x}^{\text {right }}+d_{2} I_{x x}^{\text {right }}-d_{3} I_{x y}^{\text {right }}+d_{4} \Gamma_{z x}^{r i g h t}=\Gamma_{z x}^{\text {left }} \\
& d_{1} S_{y y}^{\text {right }}+d_{2} I_{x y}^{\text {right }}-d_{3} I_{y y}^{\text {right }}+d_{4} \Gamma_{z y}^{\text {right }}=\Gamma_{z y}^{\text {left }} \\
& d_{1} S_{z z}^{\text {right }}+d_{2} \Gamma_{z x}^{\text {right }}-d_{3} \Gamma_{z y}^{\text {right }}+d_{4} \Gamma_{z z}^{r i g h t}=\Gamma_{z z l r}
\end{aligned}
$$

where the integration constants of the beam cross section, $A, S_{x x}, S_{y y}, S_{z z}, I_{x x}, I_{x y}, I_{y y}, \Gamma_{z z}, \Gamma_{z x}$ and $\Gamma_{z y}$ are the same as defined earlier after the equilibrium equations but they are now defined separately for the left and right parts of the beam cross sections. Another constant, undefined earlier, is $\Gamma_{z z l r}=\int_{A} \psi^{\text {left }} \psi^{\text {right }} d A$ which utilizes the warping function on the left and right hand side cross sections.

Here, it is noted that all integration constants are calculated using the shear centers as references for both cross-sections. At this step, the constants obtained using the pole as the references cannot be used because of the flexural-torsional coupling. However, in order to obtain the unique values of all constants no matter which element is assumed to be on the left-hand or the right-hand side, an additional normalization needs to be implemented. It requires that the product of both constants $d_{4}$-- one when the closed cross section is on the left and the other when the closed cross section is on the right -- be normalized, which is basically equivalent to the orthogonality as explained in [130]. The compatibility constants can be implemented into the model directly as follows:

$$
\begin{aligned}
&\left.u\right|_{\text {left }}=\left.u\right|_{\text {right }} \\
&\left.v\right|_{\text {left }}=\left.v\right|_{\text {right }} \\
&\left.w\right|_{\text {left }}+\left.d_{1} \phi\right|_{\text {left }}=\left.w\right|_{\text {right }} \\
&\left.\theta_{x}\right|_{\text {left }}+\left.d_{2} \phi\right|_{\text {left }}=\left.\theta_{x}\right|_{\text {right }}
\end{aligned}
$$




$$
\begin{aligned}
&\left.\theta_{y}\right|_{\text {left }}-\left.d_{3} \phi\right|_{\text {left }}=\left.\theta_{y}\right|_{\text {right }} \\
&\left.\theta_{z}\right|_{\text {left }}=\left.\theta_{z}\right|_{\text {right }} \\
&\left.d_{4} \phi\right|_{\text {left }}=\left.\phi\right|_{\text {right }} \\
&\left.P_{x}\right|_{\text {left }}=\left.P_{x}\right|_{\text {right }} \\
&\left.P_{y}\right|_{\text {left }}=\left.P_{y}\right|_{\text {right }} \\
&\left.P_{z}\right|_{\text {left }}=\left.P_{z}\right|_{\text {right }} \\
&\left.M_{x}\right|_{\text {left }}=\left.M_{x}\right|_{\text {right }} \\
&\left.M_{y}\right|_{\text {left }}=\left.M_{y}\right|_{\text {right }} \\
&\left.M_{z}\right|_{\text {left }}=\left.M_{z}\right|_{\text {right }} \\
&-\left.M_{\omega}\right|_{\text {left }}=\left.d_{1} P_{z}\right|_{\text {right }}+\left.d_{2} M_{x}\right|_{\text {right }}+\left.d_{3} M_{y}\right|_{\text {right }}-\left.d_{4} M_{\omega}\right|_{\text {right }}
\end{aligned}
$$

\subsection{Analytical Solutions}

\subsubsection{Solution Approach}

To obtain the analytical solution for the general case, we require rewriting the set of equations as a set of coupled first order equations using state variables and then use Jordan canonical form of the system to uncouple the system. For this, the following auxiliary variables: $u_{1}=u^{\prime}$, $v_{1}=v^{\prime}, \theta_{x 1}=\theta_{x}^{\prime}, \theta_{y 1}=\theta_{y}^{\prime}, \theta_{z 1}=\theta_{z}^{\prime}$ and $\phi_{1}=\phi^{\prime}$ are introduced where ( $)^{\prime}$ denotes the derivative with respect to $z ; w$ is automatically uncoupled and is, thus, not included in the analysis. The state vector of variables is defined as follows:

$$
\underline{X}=\left\lfloor\begin{array}{llllllllllll}
u & u_{1} & v & v_{1} & \theta_{x} & \theta_{x 1} & \theta_{y} & \theta_{y 1} & \theta_{z} & \theta_{z 1} & \phi & \phi_{1}
\end{array}\right\rfloor^{T}
$$

With the assignment of auxiliary variables as indicated above, the coupled equations can be rewritten in the state form as follows: 


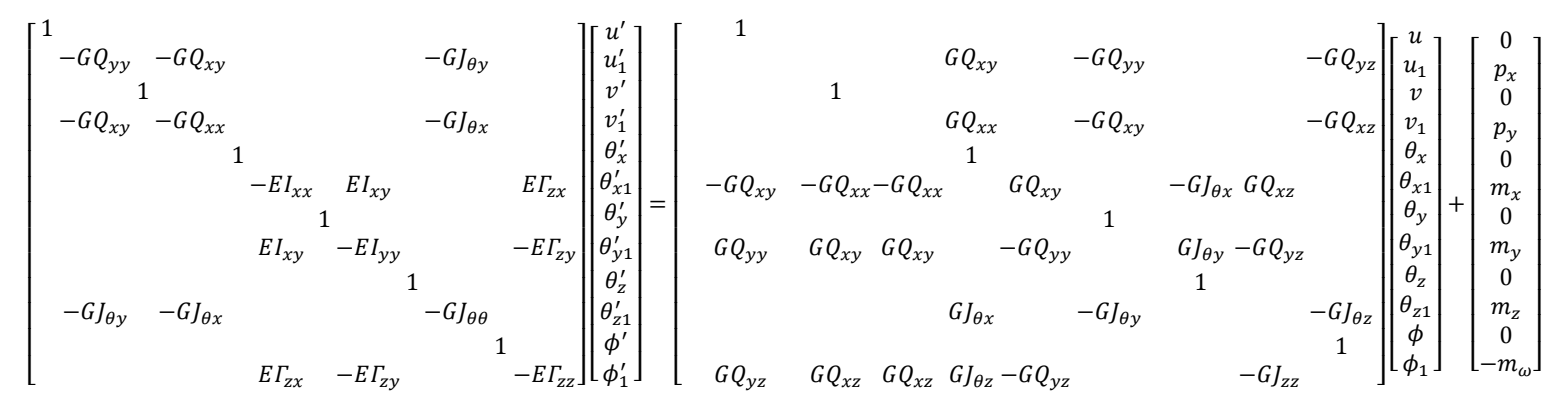

Equation (2.52) yields the $1^{\text {st }}$ order ODE equation in the following form:

$$
\begin{gathered}
\underline{A} \underline{X^{\prime}}=\underline{B} \underline{X}+\underline{F} \\
\underline{X^{\prime}}=\underline{A}^{-1} \underline{B} \underline{X}+\underline{A}^{-1} \underline{F}
\end{gathered}
$$

To obtain the solution, the technique of Jordan canonical form [132] is applied. The Jordan matrix and the corresponding matrix of transformation are represented by $J$ and $\Phi$, respectively. Let's define

$$
\underline{X}=\underline{\Phi} \underline{Q}
$$

Substitute Eq. (2.55) into Eq. (2.54), we obtain:

$$
\underline{\Phi} \underline{Q}^{\prime}=\underline{A}^{-1} \underline{B} \underline{\Phi} \underline{Q}+\underline{A}^{-1} \underline{F}
$$

Pre-multiply Eq. (2.56) by $\underline{\Phi}^{-1}$, we obtain:

$$
\underline{\Phi}^{-1} \underline{\Phi} \underline{Q}^{\prime}=\underline{\Phi}^{-1} \underline{A}^{-1} \underline{B} \underline{\Phi} \underline{Q}+\underline{\Phi}^{-1} \underline{A}^{-1} \underline{F}
$$

Consequently,

$$
\underline{Q}^{\prime}=\underline{J} \underline{Q}+\underline{E}
$$

where $\underline{\Phi}^{-1} \underline{\Phi}=\underline{I}, \underline{\Phi}^{-1} \underline{A}^{-1} \underline{B} \underline{\Phi}=J$ and $\underline{\Phi}^{-1} \underline{A}^{-1} \underline{F}=\underline{E}$. 
The final solution of ODE in Eq. (2.58) is typically written as the combination of homogeneous and particular solution as follows:

$$
\underline{X}=\underline{X}_{P}+\underline{X}_{H}
$$

The particular and homogeneous solution in this equation can be expressed as follows:

$$
\begin{gathered}
\underline{X}_{P}=\underline{\Phi} \underline{M(z)} \int_{0} \frac{M(z)^{-1}}{\underline{E}} d z \\
\underline{X}_{H}=\underline{\Phi} \underline{M(z)} \underline{c}
\end{gathered}
$$

where $\underline{M(z)}$ and $\underline{c}$ are the matrix of functions and the vector of the constants of integration, respectively. The details of various matrices and vectors are provided in Appendix A. Substituting these forms of particular and homogeneous solutions in Eq. (2.59), we obtain the following for the complete solution:

$$
\underline{X}=\underline{\Phi}[\underline{I(z)}+\underline{d(z)}]
$$

where $\underline{I(z)}=\underline{M(z)} \int_{0} \underline{M(z)^{-1}} \underline{E} d z$ and $\underline{d(z)}=\underline{M(z)} \underline{c}$.

Using this solution all response quantities related to the state vector can thus be written in analytical form. For example, the angle of twist and deflection in the $i^{\text {th }}$ beam section are respectively given as:

$$
\begin{aligned}
& \theta_{z}^{(i)}=\sum_{k=1}^{n} \Phi_{7 k}^{(i)}\left[I_{k}^{(i)}(z)+d_{k}^{(i)}(z)\right] \\
& u^{(i)}=\sum_{k=1}^{n} \Phi_{1 k}^{(i)}\left[I_{k}^{(i)}(z)+d_{k}^{(i)}(z)\right]
\end{aligned}
$$

where $n$ is the dimension of the state equation which in this case are $n=12$. 
To obtain the constants of integration in vector $\underline{c}$, we need to apply the boundary and continuity conditions in each portion of the beam. For each section, the vector $\underline{c}$ consists of 12 constants of integration. Thus, for a three section beam there are 36 such constants of integration. In addition there are 4 constants, $d_{i}$ that were introduced in enforcing the warping displacement compatibilities at each section interface. With 2 section interfaces, this introduced 8 more constant to be calculated. Thus a three section beam will require the calculation of a total of 44 constants. The 6 boundary equations, Eq. (2.17) to Eq. (2.19) at the fixed end, 6 boundary equations, Eq. (2.20) to Eq. (2.26) excluding Eq. (2.22) at the free end, 4 minimization conditions, Eq. (2.34) to Eq. (2.37) at each interface and 12 additional conditions in equations, Eq. (2.38) to Eq. (2.51) excluding Eq. (2.40) and Eq. (2.47) at each interface provide just the right number of equations to provide the all constants of integration in vector $\underline{c}$. Application of these boundary conditions leads to the following simultaneous equations to solve for the constants.

$$
\underline{G} \underline{c}=\underline{F_{G}}
$$

The details of the elements of the matrix $\underline{G}$ and vectors $\underline{c}$ and $\underline{F}_{G}$ in Eq. (2.65) are provided in the Appendix B. Knowing these constants, one can obtain any quantity of interest that can be defined in terms of the state vectors. This expression is implemented into the probabilistic analysis in the following section.

\subsubsection{Probabilistic Analysis}

In this section, the safety index approach is utilized to evaluate the effect of the presence of a crack on the system performance in probabilistic terms. It is assumed that the crack length $L_{d}$ and its location $L_{c}$ along the beam are random quantities defined by two independent random variables. It could also be assumed that other problem parameters such as cross sectional dimensions and the applied load to be random quantities. However, to simplify the formulation and to demonstrate the methodology, it is assumed that just these two crack parameters are random variables. To evaluate probabilistic performance, a limit state is defined for which the performance of the system is examined. The limit state could be defined in terms of a stress or a deformation quantity exceeding the allowable values. Herein, for demonstration, the failure limit 
state is defined in terms of the angle of twist at the free end in the damaged beam exceeding by a fraction, $\alpha$, of the angle of twist at same point in the uncracked beam, $\bar{\theta}^{\text {tip }}$. This condition can thus be stated as $\theta^{\text {tip }}>(1+\alpha) \bar{\theta}^{\text {tip }}$. The corresponding limit state boundary for safety analysis is then defined as follows:

$$
g\left(L_{c}, L_{d}\right)=(1+\alpha) \bar{\theta}^{t i p}-\theta^{t i p}
$$

where $g\left(L_{c}, L_{d}\right)>0$ implies safety and $g\left(L_{c}, L_{d}\right) \leq 0$ as the failure. The angle of twist at the tip of the cracked beam, $\theta^{\text {tip }}$, is defined by Eq. (2.63) above.

To obtain the probability of failure or survival, the probability distributions of the variables that define the limit state are required to find out the probability mass over the corresponding domains. Often, however, it is not possible to have reliable information about the distribution of the variables and only the first two moments (mean and variance) can be reliably obtained. Even if the distribution information were available, it is often very difficult to calculate the probability mass over the survival or failure domains, if the limit state is nonlinear. Methods have been developed to improve the accuracy for such calculations. The main difficulty, however, remains in defining the distributions of the random variables. With the limited information available about the first two moments, one can, however, obtain a good measure about the reliability or failure in terms of safety index. If the involved variables can also be considered to be Gaussian random variables, then the safety index provides the exact values of the probabilities if the limit state boundary is linear and an approximate value of the probabilities if the limit state boundary is nonlinear. Literature is now available that describes the safety index approach and the calculation of the probabilities. In the following, it is assumed that the mean and standard deviation values of the two random variables that define the crack parameters are known. The goal is to obtain the safety index and the approximate probability of failure assuming Gaussian distribution.

To calculate the safety index conveniently, the well-known Rackwitz and Fiessler [61-63] iterative algorithm is implemented. This algorithm calculates the design point, which is the 
closest point to the limit state boundary in the reduced space of the variables. The coordinates of the design point in the space of the variables are defined as follows:

$$
L_{c}^{*}=\mu_{c}+\beta \alpha_{c} \sigma_{c}, L_{d}^{*}=\mu_{d}+\beta \alpha_{d} \sigma_{d}
$$

where an asterisk denotes the design point value of the variables; $\mu_{c}$ and $\sigma_{c}$ are the mean and standard deviation values of the crack location, $L_{c} ; \mu_{d}$ and $\sigma_{d}$ are mean and standard deviation values of the of crack length, $L_{d} ; \beta$ is the safety index. $\alpha_{c}$ and $\alpha_{d}$ are direction cosines of the perpendicular to the limit state at the design point. These direction cosines are defined as follows:

$$
\begin{aligned}
& \alpha_{c}=\left.\frac{-\sigma_{c} \frac{\partial g}{\partial L_{c}}}{\sqrt{\sigma_{c}^{2}\left(\frac{\partial g}{\partial L_{c}}\right)^{2}+\sigma_{d^{2}}\left(\frac{\partial g}{\partial L_{d}}\right)^{2}}}\right|_{L_{c}^{*}, L_{d}^{*}} \\
& \alpha_{d}=\left.\frac{-\sigma_{d} \frac{\partial g}{\partial L_{d}}}{\sqrt{\sigma_{c}{ }^{2}\left(\frac{\partial g}{\partial L_{c}}\right)^{2}+\sigma_{d}^{2}\left(\frac{\partial g}{\partial L_{d}}\right)^{2}}}\right|_{L_{c}^{*}, L_{d}^{*}}
\end{aligned}
$$

The safety index, as the shortest distance in the reduced space, is thus defined as follows:

$$
\beta=\left[\left(\left(L_{c}^{*}-\mu_{c}\right) / \sigma_{c}\right)^{2}+\left(\left(L_{d}^{*}-\mu_{d}\right) / \sigma_{d}\right)^{2}\right]
$$

For nonlinear limit states, the calculation of the safety index is an iterative process in which appropriate values are assumed for the design point at which the gradients and direction cosines are calculated. The design point defined by Eq. (2.67) lies on the limit state. Thus substitution of Eq. (2.67) in the limit state and solving for the safety index provides the first value of the safely index. This value is used in Eq. (2.67) to obtain the new design point and the process is then repeated. The convergence is fast in most cases. The gradients of the limit state function required in Eq. (2.68) and Eq. (2.69) are obtained by the chain rule of differentiation. For example, the 
partial derivatives of the limit state function with respect to the variables $L_{c}$ and $L_{d}$ required in Eq. (2.68) and Eq. (2.69) are defined as follows:

$$
\begin{gathered}
\frac{\partial g}{\partial L_{c}}=-\frac{\partial \theta^{t i p}}{\partial L_{c}}=-\sum_{m=1}^{3} \frac{\partial \theta^{t i p}}{\partial l_{m}} \frac{\partial l_{m}}{\partial L_{c}} \\
\frac{\partial g}{\partial L_{d}}=-\frac{\partial \theta^{t i p}}{\partial L_{d}}=-\sum_{m=1}^{3} \frac{\partial \theta^{t i p}}{\partial l_{m}} \frac{\partial l_{m}}{\partial L_{d}}
\end{gathered}
$$

where $l_{1}, l_{2}$ and $l_{3}$ are the variables of the coordinates of ends of the three beam sections shown in Figure 2.1. These coordinates can be expressed in terms of the random parameters of the crack, $L_{c}$ and $L_{d}$ as follows:

$$
l_{1}=L_{c}-\frac{L_{d}}{2}, l_{2}=L_{d}, l_{3}=l-L_{c}+\frac{L_{d}}{2}
$$

The partial derivatives appearing in Eqs. (2.71) and (2.72) are defined in terms of the following partials used in the chain rule.

Derivatives of $l_{m}, \frac{\partial l_{m}}{\partial L_{c}}, \frac{\partial l_{m}}{\partial L_{d}} ; m=1,2,3$ :

$$
\begin{gathered}
\frac{\partial l_{1}}{\partial L_{c}}=1, \frac{\partial l_{2}}{\partial L_{c}}=0, \frac{\partial l_{3}}{\partial L_{c}}=-1 \\
\frac{\partial l_{1}}{\partial L_{d}}=-\frac{1}{2}, \frac{\partial l_{2}}{\partial L_{d}}=1, \frac{\partial l_{3}}{\partial L_{d}}=-\frac{1}{2}
\end{gathered}
$$

With the angle of twist at the tip defined as follows:

$$
\theta_{z}^{t i p}=\theta_{z}^{(3)}\left(l_{3}\right)=\sum_{k=1}^{n} \Phi_{9 k}^{(3)}\left[I_{k}^{(3)}\left(l_{3}\right)+d_{k}^{(3)}\left(l_{3}\right)\right]
$$

Its partial derivatives with respect to $l_{m}$ are defined as follows:

$$
\frac{\partial \theta_{z}^{(3)}\left(l_{3}\right)}{\partial l_{m}}=\sum_{k=1}^{n}\left\{\frac{\partial \Phi_{9 k}^{(3)}}{\partial l_{m}}\left[I_{k}^{(3)}\left(l_{3}\right)+d_{k}^{(3)}\left(l_{3}\right)\right]+\Phi_{9 k}^{(3)}\left(\frac{\partial I_{k}^{(3)}\left(l_{3}\right)}{\partial l_{m}}+\frac{\partial d_{k}^{(3)}\left(l_{3}\right)}{\partial l_{m}}\right)\right\}
$$


It can be shown that all elements of Jordan matrix and transition matrix in different segments are not function of $l_{m}$. Thus,

$$
\frac{\partial \Phi_{9 k}^{(3)}}{\partial l_{m}}=0 ; \quad \frac{\partial \theta_{z}^{(3)}\left(l_{3}\right)}{\partial l_{m}}=\sum_{k=1}^{n}\left\{\Phi_{9 k}^{(3)}\left(\frac{\partial I_{k}^{(3)}\left(l_{3}\right)}{\partial l_{m}}+\frac{\partial d_{k}^{(3)}\left(l_{3}\right)}{\partial l_{m}}\right)\right\}
$$

Derivatives $\frac{\partial I_{k}^{(m)}}{\partial l_{m}}$ and $\frac{\partial d_{k}^{(m)}}{\partial l_{m}}$ depend upon the matrix of functions associated with Jordan canonical form.

\subsection{Numerical Results}

\subsubsection{Structural Response}

In this section, we present some numerical results for an example problem. It is assumed that the crack is symmetrically placed in longitudinal direction and all three portions of the beam have at least one fold of span-wise symmetry about $y-z$ plane. For this one-fold symmetry, several geometric parameters of the cross section, such as $Q_{x y}, I_{x y}, J_{\theta x}, J_{\theta y}$ and $Q_{x z}$, are zero. The contour origin is taken on the axis of symmetry thus the warping displacement of the contour origin can be dropped. This means that the points on the contour origins along the span do not warp due to the fact that every point along the fold of symmetry is fixed. This simplifies the equation considerably to the following set.

$$
\begin{gathered}
-G Q_{y y}\left(u^{\prime \prime}-\theta_{y}^{\prime}\right)=p_{x} \\
-E \Gamma_{z y} \phi^{\prime \prime}-E I_{y y} \theta_{y}^{\prime \prime}-G Q_{y y}\left(u^{\prime}-\theta_{y}\right)=m_{y} \\
-G J_{\theta \theta} \theta_{z}^{\prime \prime}+G J_{\theta z} \phi^{\prime}=m_{z} \\
-E \Gamma_{z z} \phi^{\prime \prime}-E \Gamma_{z y} \theta_{y}^{\prime \prime}-G J_{\theta z} \theta_{z}^{\prime}+G J_{z z} \phi=-m_{\omega}
\end{gathered}
$$

From this set the equation of the beam displacement along the $y-y$ and $z-z$ axis are omitted, as they are uncoupled from this set and can be separately integrated. This reduced set of equations can be readily decoupled to obtain the analytical solution for each degree of freedom, as long as all distributed loadings are described as integrable functions. 
To obtain the numerical results, the thin-walled beam is assumed to be $6 \mathrm{~m}$ long, with cross sectional dimensions of: width $=0.4 \mathrm{~m}$, depth $=0.2 \mathrm{~m}$ and thickness $=0.01 \mathrm{~m}$. The modulus of elasticity is assumed to be $E=71.7 \mathrm{GPa}$ and $v=0.33$. The numerical expressions of warping functions used herein are as follows:

$$
\begin{gathered}
\psi_{c}(s)= \begin{cases}-0.0067+0.033(-0.4-s), & -0.6 \leq s<-0.4 \\
0.0067-0.067(-0.2-s), & -0.4 \leq s<-0.2 \\
-0.033 s \quad,-0.2 \leq s<0.2 \\
-0.0067+0.067(-0.2+s), & 0.2 \leq s<0.4 \\
0.0067-0.033(-0.4+s), & 0.4 \leq s<0.6\end{cases} \\
\psi_{o}(s, n)= \begin{cases}-0.012-0.34(-s-0.4)-n(s+0.6), & -0.6 \leq s<-0.4 \\
0.028-0.2(-s-0.2)-n(s+0.06), & -0.4 \leq s<-0.2 \\
-0.14 s-n s & ,-0.2 \leq s<0.2 \\
-0.028+0.2(s-0.2)-n(s-0.06), & 0.2 \leq s<0.4 \\
0.012+0.34(s-0.4)-n(s-0.6), & 0.4 \leq s<0.6\end{cases}
\end{gathered}
$$

These warping functions are shown in meters in Figure 2.3.

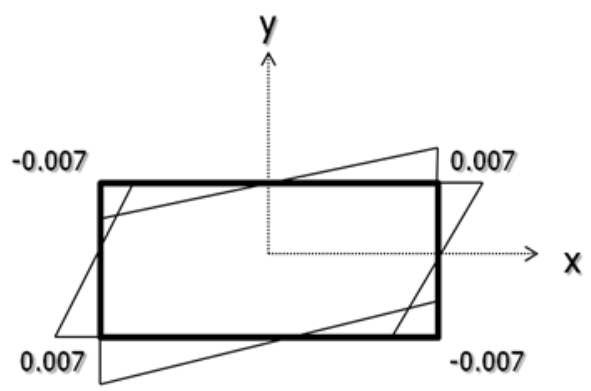

a) Closed cross-section

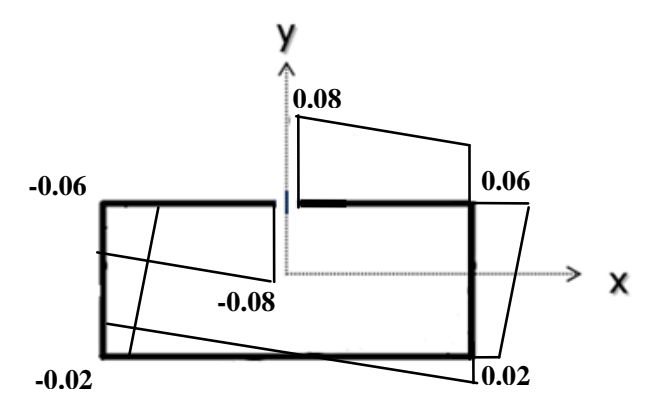

b) Open cross-section

Figure 2.3 Numerical plots of warping functions 
For this particular section, it is found that the compatibility constants have the following values, $d_{1}=d_{2}=0, d_{3}=-0.0294$, and $d_{4}=0.122$, the open cross-section being on the right-hand side. $d_{3}=0.240$ and $d_{4}=8.16$ when the open cross-section is on the left-hand side.

To validate this applied beam theory, the numerical results of a cracked beam obtained by this approach are compared with the more accurate results obtained computationally by the shell theory. The details of the shell model are provided in the Appendix C. The numerical results are obtained and compared for the angle of twist and lateral deflection of three beams with three different crack sizes. The beams are subjected to the combined loadings of a torque, $M_{z}=1$ $N \cdot m$ and a lateral shear force, $P_{x}=1 N$, applied at the beam tip. Figure 2.4 shows the comparison of the angle of twist values and Figure 2.5 for the lateral deflection values for the three cases.

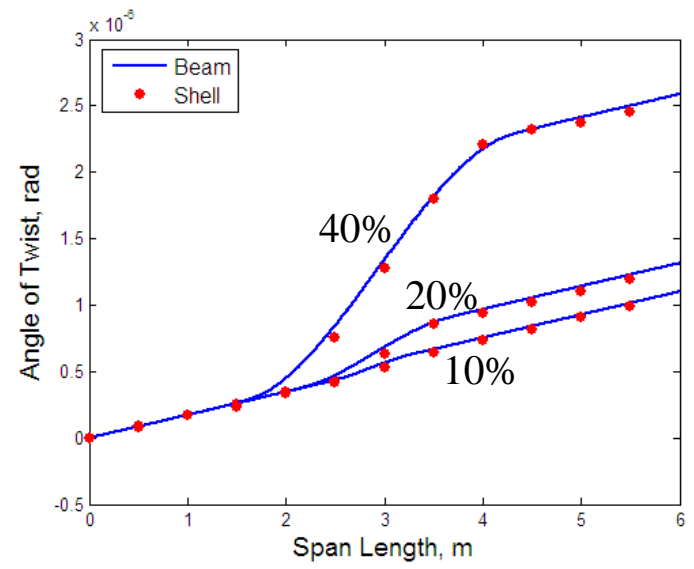

Figure 2.4 Angle of twist along the beam span

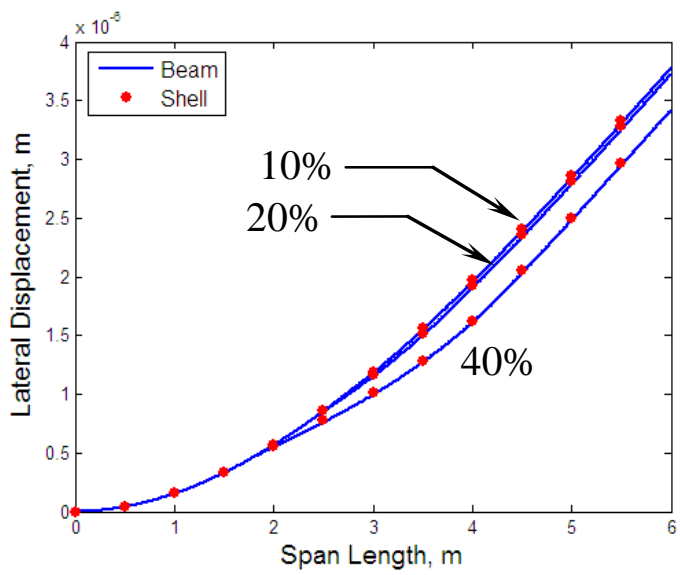

Figure 2.5 Lateral deflection along the beam span 
The results clearly show that even for large cracks the proposed beam model yields quite consistent results for both the twist angle and deflection compared to those obtained with shell finite elements in ABAQUS ${ }^{\circledR}$, thus validating the accuracy of the analytical solution.

\subsubsection{Probabilistic Safety Analysis}

In the following, we show the results for crack parameters assumed to be Gaussian random variables with the mean and standard deviation values taken as $L_{c}: N(3.0,0.5)$ for the random variable of the crack location and $L_{d}: N(0.6,0.15)$ for the random variable of the crack size. The value of fraction $\alpha$ in Eq. (2.66) is assumed to be $\alpha=0.1$. In order to investigate the effects from different loads, a study is conducted for three different cases of distributed loadings, a) Uniform distribution, b) Triangle shape and c) Quarter-ellipse shape as depicted in Figure 2.6.

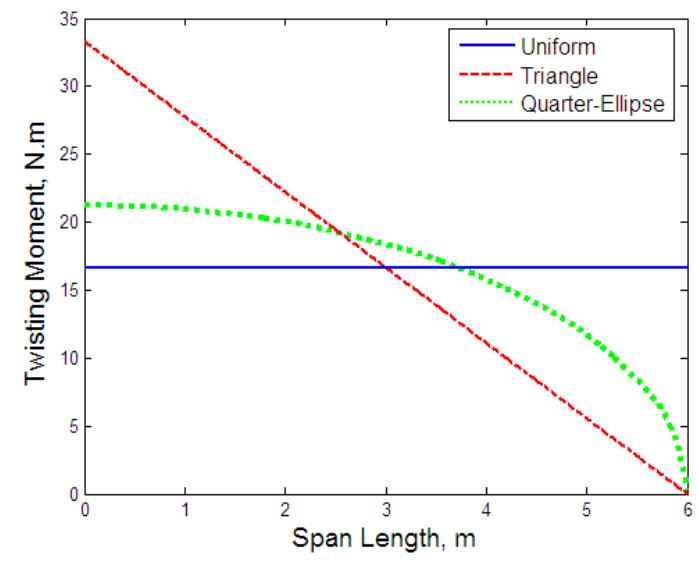

Figure 2.6 Distributed twisting moment along the beam span

For comparison purpose, the areas under all shapes of loadings are set to be identical and equal $100 \mathrm{~N} \cdot \mathrm{m}$; however, due to the fact that the relative condition for limit state function is used as previously illustrated, the magnitude of this area does not matter to the probabilistic results. To obtain the values of the safety index, $\beta$, for such failure criteria, the iterative approach proposed by Rackwitz and Fiessler [63] is used. Since the limit state function is only implicitly defined in terms of the random variables $L_{c}$ and $L_{d}$, the gradients of the limit state boundaries with respect to these variables are calculated by using the chain rule. The design point and safety index values converged in three iterations for all three loading cases. The final values for the design point, safety index and probability of non-exceedance of the limit states are shown in 
Table 2.1 for this case. As both variables are Gaussian, the probability of failure is simply defined as $p_{f}=1-\Phi(\beta)$. As one would expect, the safety index value is the lowest for the uniform loading and highest for the triangular loading; the reverse is also true for the probability of failure values.

Table 2.2 shows the comparisons of the probability of failure values computed by the above safety index approach and the Monte Carlo simulation. The results obtained by the proposed approach are based on three cycles of iteration while those from the Monte Carlo approach were obtained using 500 simulations. The results demonstrate the consistency as well as the validation of this approach. The table also provides the computation time required by these two methods on the same computer, showing the relative computational efficiency of the safety index approach.

\begin{tabular}{|c|c|c|c|c|}
\hline \multirow{2}{*}{ Loading type } & \multicolumn{2}{|c|}{ Design point values } & \multirow{2}{*}{$\begin{array}{l}\text { Safety } \\
\text { index }\end{array}$} & \multirow{2}{*}{$\begin{array}{c}\text { Probability of } \\
\text { failure }\end{array}$} \\
\hline & Location, $L_{c}^{*}$ & Size, $L_{d}^{*}$ & & \\
\hline Uniform & 2.8268 & 0.74272 & 1.0125 & $15.6 \%$ \\
\hline Quarter-ellipse & 2.7414 & 0.75526 & 1.1571 & $12.4 \%$ \\
\hline Triangular & 2.5841 & 0.78151 & 1.4684 & $7.10 \%$ \\
\hline
\end{tabular}

In Figure 2.7, we show the values of the safety index and the corresponding probabilities of failure for the case of quarter-ellipse loading for a crack location defined by parameters $L_{c}$ : $N(3.0,0.5)$ and for three crack sizes with mean values of $10 \%, 15 \%$ and $20 \%$ and increasing coefficients of variation. For this figure, the limit state is defined for $\alpha=0.3$. As expected, the safety index values decrease and the probability of failure values increase with the increasing coefficient of variation values. For a mean crack size of $21.0 \%$, the probability of failure is 0.5 and the value of safety index is zero. We call this set of value as the critical mean crack size values. This critical crack size depends upon the value of the parameter $\alpha$. For this critical value, the limit state boundaries pass through the origin of the reduced space. For any mean crack size values higher than the critical values, the safety index values are negative. Also in such cases, the safety index and the corresponding probability of failure value decreases with the increase in the 
coefficient of variation. This is because as the coefficient of variation value increases, the chances of the crack length approaching the critical length also increase; thus, causing the probability of failure to increase to 0.5 . These counter intuitive cases are, however, not presented here.

Table 2.2 Comparison of probability of failure values calculated by Rackwitz-Fiessler algorithm and Monte Carlo simulation approach

\begin{tabular}{cccc}
\hline Loading & Methodology & $\begin{array}{c}\text { Probability of } \\
\text { failure }(\%)\end{array}$ & $\begin{array}{c}\text { Computation time } \\
\text { (second) }\end{array}$ \\
\hline Uniform & Proposed Method & 15.6 & 31.9 \\
& Monte Carlo simulation & 17.4 & 768 \\
Quarter-ellipse & Proposed Method & 12.4 & 339 \\
& Monte Carlo simulation & 11.9 & 2,380 \\
Triangle & Proposed Method & 7.10 & 58.8 \\
& Monte Carlo simulation & 7.30 & 826 \\
\hline \hline
\end{tabular}

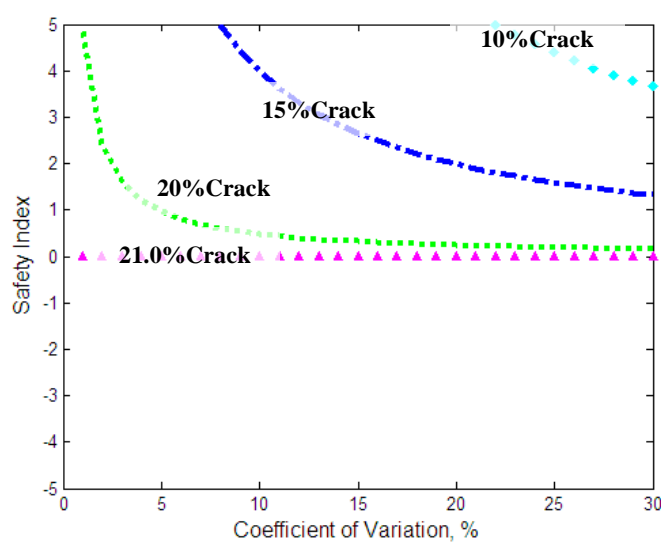

a) Safety index

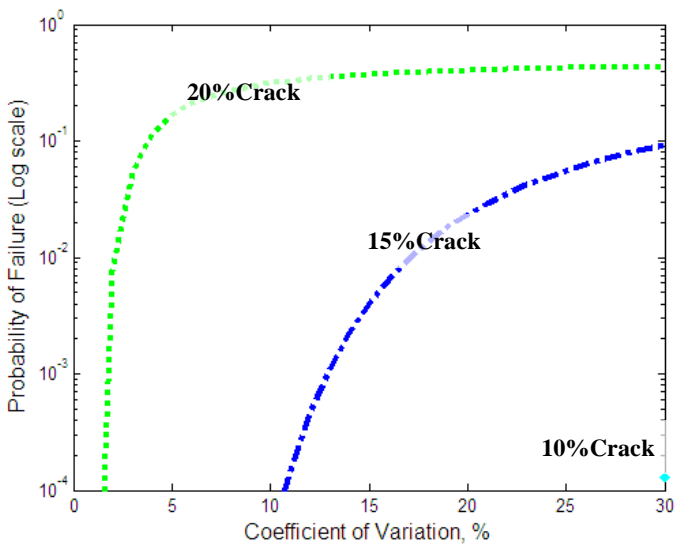

b) Probability of failure

Figure 2.7 Plots of safety indices and probabilities of failure versus coefficient of variation for $\alpha=0.3$, quarter-ellipse loading and different mean crack sizes

In the next two sets of figures, we compare the effect of the three loading patterns on the safety index and probability of failure values for increasing values of the coefficient of variation of the crack size. The mean location of the crack is in the middle of the beam -- $3 \mathrm{~m}$ from the 
fixed end. The mean crack size for Figure 2.9 is $15 \%$ of the beam length and for Figure 2.10 is for $20 \%$ of the beam length. Although the total torque on the beam is same for the three loading distributions, the uniform loading is the most severe of the three loadings as it leads to the lowest set of safety index values and the highest set of probability of failure values.

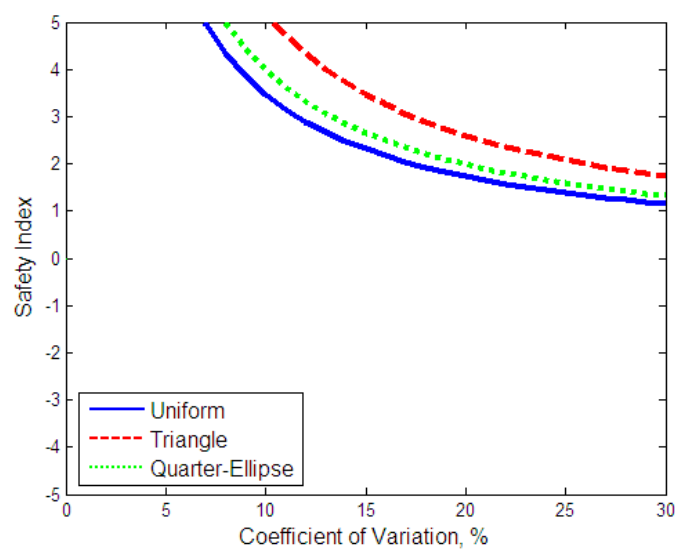

a) Safety index

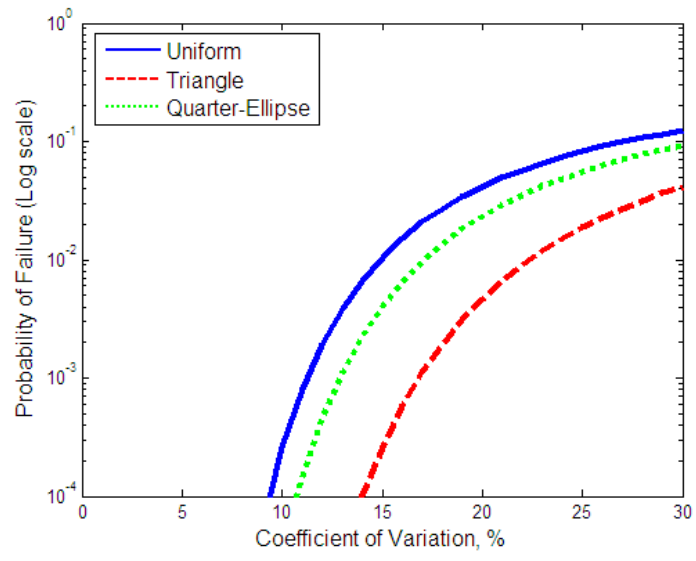

b) Probability of failure

Figure 2.8 Plots of safety indices and probabilities of failure versus coefficient of variation for mean crack sizes of $15 \%, \alpha=0.3$ and three different loadings

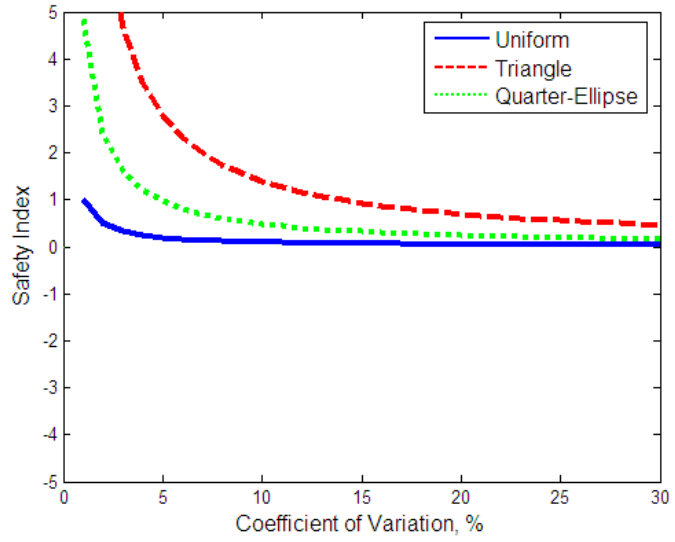

a) Safety index

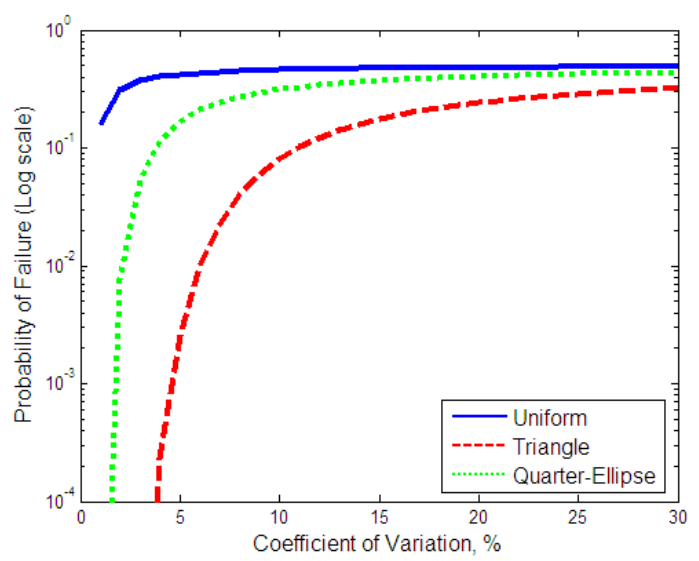

b) Probability of failure

Figure 2.9 Plots of safety indices and probabilities of failure versus coefficient of variation for mean crack sizes of $20 \%, \alpha=0.3$ and three different loadings

In Figure 2.11, we investigate the effect of different loading patterns on the safety index and probability of failure values for different locations of crack along the beam span. For the crack 
size, its mean value is $15 \%$ of the beam length and its coefficient of variation is set at $20 \%$. It is observed from the figure that both the locations of damage and the patterns of loadings do impact on the safety index and the corresponding probability of failure. For example, the safety index of the uniform loading case is higher than that of quarter-ellipse loading case when the location of damage less than a certain value around $L / 4$ from the fixed end. This trend reverses for cracks located beyond that value near $\mathrm{L} / 4$.

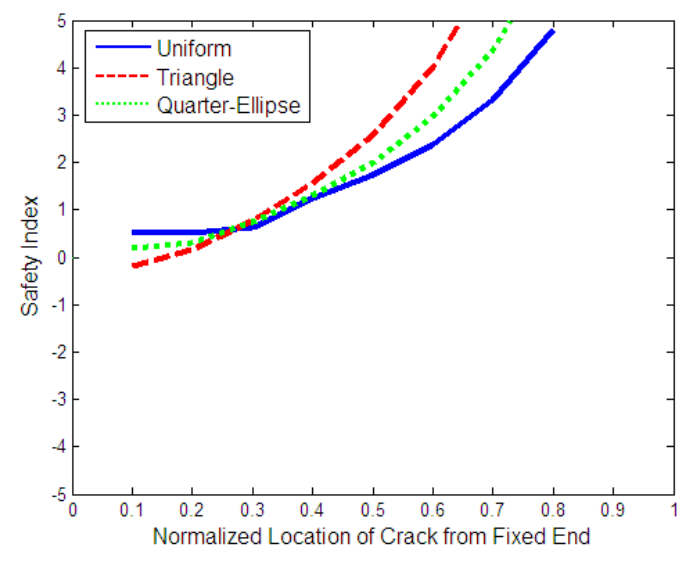

a) Safety index

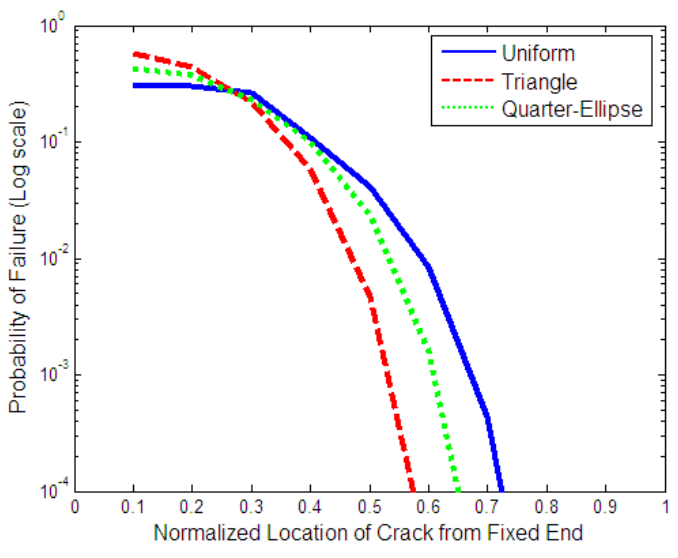

b) Probability of failure

Figure 2.10 Plots of safety indices and probabilities of failure versus location of crack for mean crack sizes of $15 \%$, coefficient of variation of $20 \%, \alpha=0.3$ and three different loadings

In Figure 2.12, we investigate the effect of different sizes of damage on the safety index and probability of failure values for different locations of crack along the beam span. These results are obtained for the loading pattern of quarter-ellipse. All crack sizes are assumed to have coefficient of variation of $20 \%$. As expected, the beam subjected to smaller damage possesses the higher safety index and lower probability of failure. Also the safety index increases and the probability of failure decreases as the crack is located away from the fixed end. 


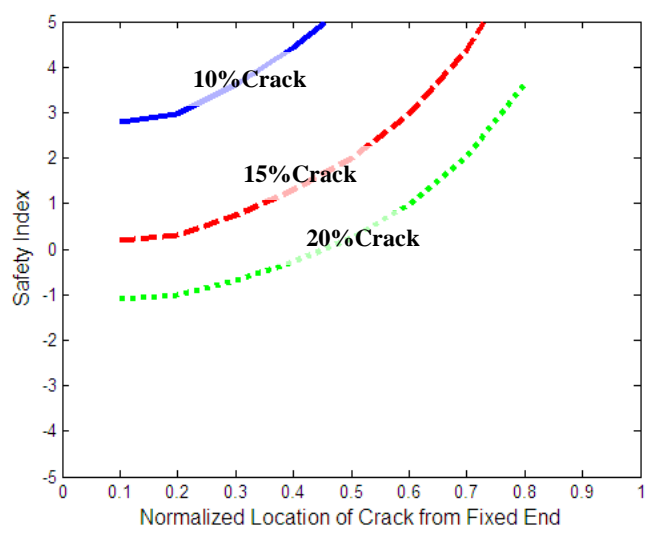

a) Safety index

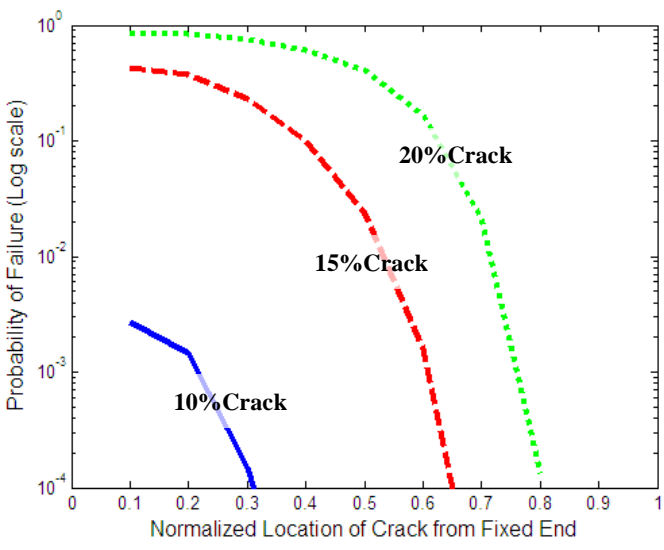

b) Probability of failure

Figure 2.11 Plots of safety indices and probabilities of failure versus location of crack for coefficient of variation of $20 \%, \alpha=0.3$, quarter-ellipse loading and two different mean crack sizes

\subsection{Summary and Concluding Remarks}

The paper presents an analytical approach to calculate the response of a thin walled beam with a crack oriented along the beam length. The governing equations and associated boundary conditions of the system are derived by variational principle. The coupled equations are then expressed in a state-space form which facilitates their uncoupling using the Jordan canonical form approach. This simple model where the response can be calculated analytically allows one to study the effect of various damage parameters in a rather simple manner. Such formulations also enable us to test the applicability of different approaches for calculating the crack growth without conducting a highly expensive finite element analysis. The analytical solution obtained with Jordan canonical form approach is used in the probabilistic analysis, assuming that crack location and crack size are random variables. First order reliability analysis is used to calculate the safety index and probability of failure for a limit state defined in terms of the angle of twist. The methodology for calculating the gradients for the limit boundary needed in the probabilistic analysis is presented. Numerical results demonstrating the application of this approach are presented for several combinations of parameters: crack size, crack size variability, crack location and different loading patterns. 
Chapter 3: Random Crack Propagation of a Thinwalled Beam with a Crack 


\author{
Random Crack Propagation of a Thin-walled Beam with a Crack ${ }^{\dagger}$ \\ Chalitphan Kunaporn, Mahendra P. Singh, Mayuresh J. Patil and Rakesh K. Kapania \\ Virginia Polytechnic Institute and State University, Blacksburg, VA 24061
}

\begin{abstract}
Probabilistic methodology is used for estimating the growth of an existing crack in a thin-walled beam with a closed cross-section. The beam is subjected to stochastic loads. The initial length of the crack is considered to be an uncertain quantity represented by a random variable. The random characteristics of the stress response, needed for crack growth analysis, are obtained by random vibration analysis of the thin-walled beam. The crack growth rate equation is solved for a large number of randomly generated sample values of the random parameters. The simulated crack growth results are then used to calculate the probability of failure. The failure is defined to occur when the actual crack size exceeds the critical crack size. For demonstration of the methodology, the numerical results for the timedependent probability of failure are obtained for an example problem of a thin-walled beam.
\end{abstract}

\title{
3.1 Introduction
}

The crack propagation approach is typically used in fatigue analysis and in predicting remaining life of structural elements subjected to cyclic loadings. In this paper, we propose to use it to predict the time to failure of a thin-walled beam assumed to represent a damaged aircraft wing. An analytical beam model can be used for repeated analyses for different loadings and damage scenarios at acceptable computational cost while a finite element model with several thousand degrees of freedom would require significant computational resources. We assume that the beam has been damaged and this damage is represented by a longitudinal crack of finite but uncertain dimensions and uncertain location along the beam.

For investigations of fatigue crack growth, a fracture mechanics based methodology was proposed in early 1960s. The approach focused on the intermediate phase of crack propagation

\footnotetext{
${ }^{\dagger}$ This article is prepared for submission to the journal of aircraft.
} 
between crack initiation and final fracture where the concept of linear elastic fracture mechanics is applicable. It is based on the assumption that a small crack can grow when it is subjected to cyclic loads [102]. The basic concept of this approach is that the crack growth rate is directly related to the change in the stress intensity factor and is in the form of an empirical differentialequation. The classical model that has been most commonly used is the Paris-Erdogan equation [103] and its variations such as the Forman equation [104] and the Walker equation [105]. These variations were introduced to obtain better fits with the experimental data. A comprehensive review of crack propagation models can be found in Beden et al. [112].

There has also been considerable work done on the topic of crack growth under stochastic loads that are commonly encountered in practice. A brief account of these studies is given in Chapter 1. These studies have led to methodologies that can be effectively applied to examine crack growth under stochastic loads such as the problem in this paper. The modified Forman equation rewritten in the time domain is applied in this study. Several parameters in the models are considered uncertain. The beam is subjected to a stochastically defined load which causes the crack to grow. For calculating random characteristics of the stress in the beam, an analytical approach based on Benscoter beam theory $[8,13]$ is used. The equations of motion are derived using the principle of virtual work. The coupled equations of motion can be solved using either exact approach such as the eigenfunction expansion approach, applied in both time and spatial domains; or using an approximate approach such as the Rayleigh-Ritz method with assumed modes. In this study, the assumed-mode approach is applied to discretize the continuous system.

Probabilistic characteristics of random variables involved in the analysis are obtained from the global analysis of the structure while expressions of stress intensity factor are appropriately chosen for the particular structural element. For crack propagation analysis under random loads, the random characteristics of the peak stress response are calculated to include the effect of random cycles. The differential equation of the crack growth model is solved to obtain the evolution of the crack growth with time for several sample values of the random variables involved in the model. At each time instant, the simulated crack growth curves provide data to define the probability distribution of the crack size. This probabilistically defined crack size is then probabilistically compared with the critical crack size at which the structure fails. This 
provides the probability of the structural failure at different time instants. Further details of the approach are described in the following sections.

\subsection{Crack Growth Modeling}

The probabilistic analysis of structure defines the initial condition of damage. To further investigate the effects of damage on the fatigue life of the both wings, a crack propagation analysis is used to simulate the growth of crack over time. Over many decades, models have been developed to capture the characteristics of crack growth observed in experiments. There are a number of ways to implement stochastic factors into any of these models. For engineering purposes, the crack growth model based on fracture mechanics is recommended since the fatigue physical process is more relevant in the investigation of crack growth over time.

The Paris-Erdogan model of crack propagation is used in the present study. This model has been widely used to study the fatigue crack growth in materials. It defines the rate of change of the crack size with respect to the number of stress cycle as a linear function of the stress intensity factor on the log-log scale. It is observed to fit the experimental data in the initial stages well. To describe the behavior near failure and to capture intermingling of the growth curves obtained in experiments, some modifications have been suggested. One of the modifications is by Forman $[99,126]$ which is described by the following equations.

$$
\frac{d a}{f_{s} d t}=\frac{C \Delta K^{m}}{\left(1-\frac{\Delta K}{(1-R) K_{C}}\right)^{q}} X(t)
$$

where $\Delta K$ is the change in the stress intensity factor; $K_{c}$ is the critical stress intensity factor or socalled fracture toughness; $C, m$ and $q$ are the material constants; $f_{s}$ is the equivalent loading frequency which allows to define the rate in terms of time instead of number of cycles; and $R$ is the static bias factor or stress ratio, which is defined as the ratio of the minimum to maximum stress values. The function $X(t)$ is a random process, which makes the crack growth stochastic and creates intermingling in the growth curves observed in real data [115]. For two extreme cases, Gaussian random process is assumed for totally uncorrelated and Gaussian random 
variables is assumed for fully correlated at all time. Here Gaussian random process with density function $N(1,0.1)$ is assumed and $X(t)$ is not included for the negative values of $R$.

To represent the nondeterministic nature of the crack growth, material-related parameters such as fracture toughness as well as other model parameters can be assumed to be random variables. In this study, the initial crack size is assumed to be a random variable. The failure is defined by the condition that the rate of change of crack length becomes unbounded, indicating that the crack grows uncontrollably and causes eventual failure. It is thus defined by

$$
\frac{\mathrm{da}}{\mathrm{dt}}=\infty
$$

This implies that the denominator of Eq. (3.1) approaches zero.

$$
1-\frac{\Delta K}{(1-R) K_{c}}=0
$$

The change in the stress intensity factor $\Delta K$ is a function of crack size, shape and member size. For some standard configurations, expressions for this have been developed and are available in the literature on the subject reference. For a special configuration, one may need to define this by refined numerical analysis. In this study, it is defined by the following equation.

$$
\Delta K=\Delta \sigma \sqrt{\pi a} f(a)
$$

where $\Delta \sigma$ is the stress range (the difference of the maximum and minimum stress); $a$ is the crack length; $f(a)$ is the geometric factor. For the particular case of a thin walled beam with a crack this factor as developed by Dang et al. [133] is expressed as follows:

$$
f(a)=\sqrt{1+2.52\left(\frac{a}{b}\right)^{2}-1.2089\left(\frac{a}{b}\right)^{4}} ; \frac{a}{b} \leq 1
$$

where $b$ is the width of the rectangular cross section. 
To obtain the critical crack length, one can solve Eq. (3.3). The probability of failure of the structure is defined when the crack size becomes such that Eq. (3.2) is satisfied. That is, when the rate of the crack growth becomes uncontrollably large.

One needs to solve Eq. (3.1) to define the crack length as a function of time. One generates the random values of the involved variables and generates the time function for $X(t)$ using the characteristics of the random process. One also needs to solve the vibration problem for the external random loads to define the stress level and the peak response characteristics of the stress. These stress values are used in calculating the stress ratio and stress range.

For a stochastically defined external load, the stress response as well as peak characteristics are random quantities. That is, there is no unique frequency $f_{s}$ and corresponding stress amplitude. These quantities are also defined in probabilistic terms and some characteristics values are used in Eq. (3.1). Finally, with all parameter values defined, Eq. (3.1) can be numerically solved. At each time step, the collective solutions obtained for each random sample set define the sample probability density function of the crack length.

The structural component is assumed to have failed when the crack length exceeds the critical length. The probability of failure of this event can be obtained by using the sample probability density function of the crack length and that of the critical crack length. Thus a complete crack growth analysis under random loading involves: (a) the random response analysis of the structure to calculate the peak stresses and the equivalent load frequency, (b) the numerical solution of Eq. (3.1) and (c) the calculation of probability of failure analysis at each time step that is used to predict remaining flight time.

\subsection{Dynamic Analysis of a Thin-walled Beam}

In this section, the equations of motion are first developed for a thin-walled beam of any shape of cross section. The thin-walled beam of a closed cross section is modeled by the Benscoter beam element in which an additional warping degree of freedom, independent of the angle of twist is used [13]. Boundary conditions are derived for the fixed end and the free end. 
The cracked portion being small compared to the length of span is assumed not to affect the global response and, thus, the presence of the crack is excluded from the dynamic analysis.
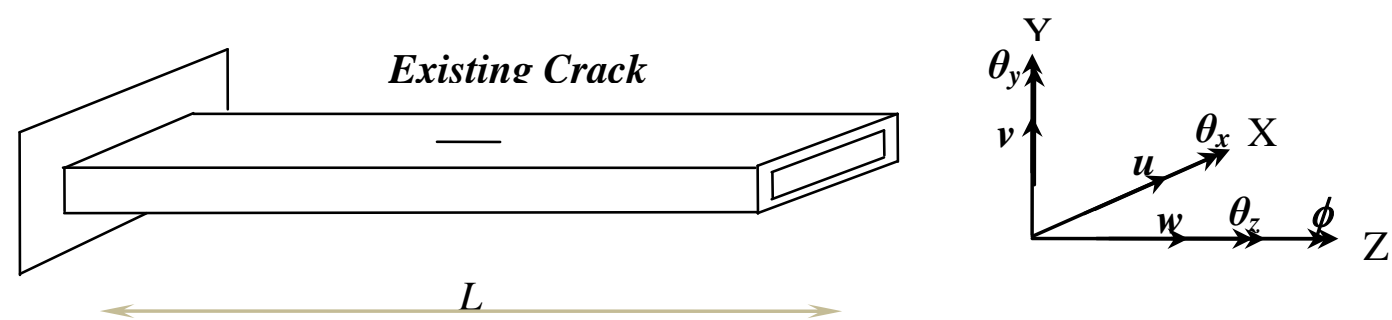

Figure 3.1 Cracked wing model based on a thin-walled cantilever

The cross-section is not deformable in its own plane and therefore only rigid body motions are allowed for in-plane displacements. An arbitrary point $P\left(x_{p}, y_{p}\right)$ called as the "Pole" is used as the reference to describe all displacement fields. The pole is chosen to coincide with the center of mass. In thin-walled theory, the two displacements that are required in the derivation of governing equations are: the tangential displacement, $v_{S}$, and the out-of-plane or warping displacement, $w$, both defined in the $(s, n, z)$ coordinate. A point $\operatorname{CO}\left(x_{c o}, y_{c o}\right)$ called as the origin of contour is used as the reference point for contour integration, $s=0$, to calculate the warping function of section.

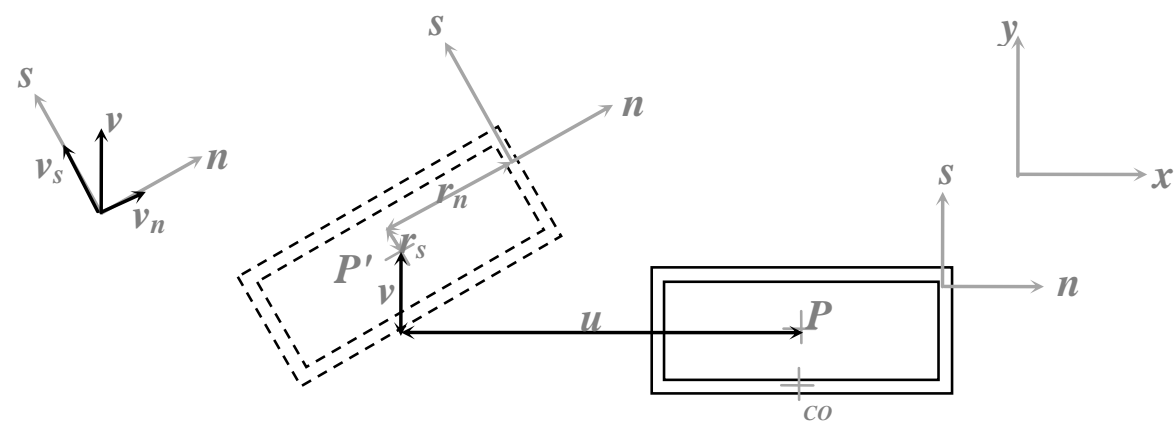

Figure 3.2 Kinematics of a beam cross-section

The additional degrees of freedom used as the measure of warping, $\phi(z)$, is assumed to be independent of the axial rotational degree of freedom, the angle and the rate of twist, of the 
beam. In terms of these degrees of freedom, the tangential and warping displacements can be written as follows [32]:

$$
\begin{gathered}
v_{s}(s, n, z)=u(z) \frac{\partial x(s)}{\partial s}+v(z) \frac{\partial y(s)}{\partial s}+r_{n}(s, n) \theta_{z}(z) \\
w(s, n, z)=w(z)+y(s) \theta_{x}(z)+x(s) \theta_{y}(z)-\psi(s, n) \phi(z)
\end{gathered}
$$

where $u(z)$ and $v(z)$ are the displacements of the pole in $x$ and $y$ directions respectively; $w(z)$ is the warping displacement of the contour origin; $\theta_{x}(z)$ and $\theta_{y}(z)$ are the rotations of pole with respect to the $x$ and $y$ axis respectively; $\theta_{z}(z)$ is the angle of twist; $\psi(s, n)$ is the warping function; $\phi(z)$ is the measure of warping that in special cases can be approximated to the rate of twist, $\theta_{z}^{\prime} ;()^{\prime}$ denotes the derivative with respect to $z ; x(s)$ and $y(s)$ are Cartesian coordinate rewritten in term of tangential coordinate; and the distance $r_{n}(s, n)$ is shown in Figure 3.2. In the linear theory, the rotations of pole are described as follows:

$$
\begin{aligned}
& \theta_{x}(z)=\gamma_{y z}(z)-v^{\prime}(z) \\
& \theta_{y}(z)=\gamma_{x z}(z)-u^{\prime}(z)
\end{aligned}
$$

where $\gamma_{x z}$ and $\gamma_{y z}$ are transverse shear strains. In the shear deformable beam theory, the EulerBernoulli hypothesis is no longer applicable as $\gamma_{x z} \neq 0$ and $\gamma_{y z} \neq 0$ and the transverse strains are included in the derivation. Infinitesimal strain tensor in linear theory is expressed in terms of displacements. Based on the thin-walled theory, the energy equation requires only two main strain components, $\varepsilon_{z z}$, which is the normal strain in $z$-direction, and $\gamma_{s z}=2 \varepsilon_{s z}$, which is the shear strain in the $s-z$ plane. These strain components are defined as follows:

$$
\begin{gathered}
\varepsilon_{z z}=\frac{\partial w}{\partial z} \\
\gamma_{s z}=2 \varepsilon_{s z}=\frac{\partial v_{s}}{\partial z}+\frac{\partial w}{\partial s}
\end{gathered}
$$

Even a beam subjected only to a twisting moment is no longer a case of pure torsion. Coupled bending and torsion must be considered. Benscoter beam element includes the warping degree of 
freedom. The governing equations of the thin-walled beam are derived using the principle of virtual work. The principle of virtual work can be written as follows:

$$
\begin{gathered}
\delta W_{\text {Internal Work }}-\delta W_{\text {External Work }}-\delta W_{\text {Inertia }}=0 \\
\iiint_{V} \sigma \delta \varepsilon d V-\left(\iiint_{V} B \delta u d V+\iint_{S} F \delta u d S\right)-\iiint_{V} \rho \ddot{\mathrm{U}} \delta \mathrm{U} d V=0
\end{gathered}
$$

where $B$ and $F$ stand for body forces and tractions, respectively.

For a beam of thin-walled cross-section, the strain energy term is simplified by deleting the terms with zero strains and considering only the non-zero strain terms. With tractions in the form of distributed loads along the span and concentrated loads at certain locations of the span, the equation of virtual work can be written as follows:

$$
\begin{aligned}
& \int_{a}^{b}\left[\int_{A}\left[E \varepsilon_{z z} \delta \varepsilon_{z z}+G \gamma_{s z} \delta \gamma_{s z}-\rho(\ddot{u} \delta u+\ddot{v} \delta v+\ddot{w} \delta w)\right] d A\right. \\
& \left.-\left(p_{x} \delta u+p_{y} \delta v+p_{z} \delta w+m_{x} \delta \theta_{y}+m_{y} \delta \theta_{x}+m_{z} \delta \theta_{z}-m_{\omega} \delta \phi\right)\right] d Z \\
& -\left(P_{x} \delta u+P_{y} \delta v+P_{z} \delta w+M_{x} \delta \theta_{x}+M_{y} \delta \theta_{y}+M_{z} \delta \theta_{z}-M_{\omega} \delta \phi\right)=0
\end{aligned}
$$

where $E$ is the Young's modulus and $G$ is the modulus of torsional rigidity; $p_{x}, p_{y}$ and $p_{z}$ are the distributed forces in $x, y$ and $z$ direction, respectively; $m_{x}, m_{y}$ and $m_{z}$ are the distributed moments with respect to $x, y$ and $z$ axis, respectively; $m_{\omega}$ is the distributed warping torque about the $z$ axis; $P_{x}$ and $P_{y}$ are the shear forces in $x$ and $y$ directions, respectively; $P_{z}$ is the axial force; $M_{x}$ and $M_{y}$ are the bending moments with respect to $x$ and $y$ axis, respectively; $M_{z}$ is the torque; and $M_{\omega}$ is the warping moment or so-called "Bimoment".

Substituting all stress and strain components and applying the principle of virtual work, the system of equilibrium equations as well as corresponding boundary-continuity conditions are obtained as follows:

$$
\begin{gathered}
-E A w^{\prime \prime}+\rho A \ddot{w}=p_{z} \\
-G J_{\theta y} \theta_{z}^{\prime \prime}+G Q_{y z} \phi^{\prime}-G Q_{x y}\left(v^{\prime \prime}+\theta_{x}^{\prime}\right)-G Q_{y y}\left(u^{\prime \prime}-\theta_{y}^{\prime}\right)+\rho\left(A \ddot{u}-S_{x x} \ddot{\theta}_{z}\right)=p_{x}
\end{gathered}
$$




$$
\begin{aligned}
& -G J_{\theta x} \theta_{z}^{\prime \prime}+G Q_{x z} \phi^{\prime}-G Q_{x x}\left(v^{\prime \prime}+\theta_{x}^{\prime}\right)-G Q_{x y}\left(u^{\prime \prime}-\theta_{y}^{\prime}\right)+\rho\left(A \ddot{v}+S_{y y} \ddot{\theta}_{z}\right)=p_{y} \\
& \quad E \Gamma_{z x} \phi^{\prime \prime}-E I_{x x} \theta_{x}^{\prime \prime}+E I_{x y} \theta_{y}^{\prime \prime}+G J_{\theta x} \theta_{z}^{\prime}-G Q_{x z} \phi+G Q_{x x}\left(v^{\prime}+\theta_{x}\right)+G Q_{x y}\left(u^{\prime}-\theta_{y}\right)+ \\
& \rho\left(I_{x x} \ddot{\theta}_{x}-I_{x y} \ddot{\theta}_{y}-\Gamma_{z x} \ddot{\phi}\right)=m_{x} \\
& \quad-E \Gamma_{z y} \phi^{\prime \prime}+E I_{x y} \theta_{x}^{\prime \prime}-E I_{y y} \theta_{y}^{\prime \prime}-G J_{\theta y} \theta_{z}^{\prime}+G Q_{y z} \phi-G Q_{x y}\left(v^{\prime}+\theta_{x}\right)-G Q_{y y}\left(u^{\prime}-\theta_{y}\right)+ \\
& \rho\left(-I_{x y} \ddot{\theta}_{x}+I_{y y} \ddot{\theta}_{y}+\Gamma_{z y} \ddot{\theta}\right)=m_{y} \\
& \quad-G J_{\theta \theta} \theta_{z}^{\prime \prime}+G J_{\theta z} \phi^{\prime}-G J_{\theta x}\left(v^{\prime \prime}+\theta_{x}^{\prime}\right)-G J_{\theta y}\left(u^{\prime \prime}-\theta_{y}^{\prime}\right)+\rho\left(\left(I_{x x}+I_{y y}\right) \ddot{\theta}_{z}-S_{x x} \ddot{u}+\right. \\
& \left.S_{y y} \ddot{v}\right)=m_{z} \\
& \quad-E \Gamma_{z z} \phi^{\prime \prime}+E \Gamma_{z x} \theta_{x}^{\prime \prime}-E \Gamma_{z y} \theta_{y}^{\prime \prime}-G J_{\theta z} \theta_{z}^{\prime}+G J_{z z} \phi-G Q_{x z}\left(v^{\prime}+\theta_{x}\right)-G Q_{y z}\left(u^{\prime}-\theta_{y}\right)+ \\
& \rho\left(\Gamma_{z z} \ddot{\phi}-\Gamma_{z x} \ddot{\theta}_{x}+\Gamma_{z y} \ddot{\theta}_{y}\right)=-m_{\omega}
\end{aligned}
$$

The geometric problem parameters used in the above equations are defined as follows:

$$
\begin{aligned}
& A=\int_{A} d A, S_{x x}=\int_{A} y d A, S_{y y}=\int_{A} x d A, S_{z z}=\int_{A} \psi d A \\
& \Gamma_{z z}=\int_{A} \psi^{2} d A, \Gamma_{z x}=\int_{A} y \psi d A, \Gamma_{z y}=\int_{A} x \psi d A \\
& I_{x x}=\int_{A} y^{2} d A, I_{x y}=\int_{A} x y d A, I_{y y}=\int_{A} x^{2} d A \\
& J_{\theta \theta}=\int_{A} R_{n}^{2} d A, J_{\theta z}=\int_{A} R_{n} \frac{\partial \psi}{\partial s} d A, J_{z z}=\int_{A}\left(\frac{\partial \psi}{\partial s}\right)^{2} d A, J_{\theta x}=\int_{A} R_{n} \frac{\partial y}{\partial s} d A, J_{\theta y}=\int_{A} R_{n} \frac{\partial x}{\partial s} d A \\
& Q_{x x}=\int_{A}\left(\frac{\partial y}{\partial s}\right)^{2} d A, Q_{x y}=\int_{A} \frac{\partial x}{\partial s} \frac{\partial y}{\partial s} d A, Q_{y y}=\int_{A}\left(\frac{\partial x}{\partial s}\right)^{2} d A, Q_{x z}=\int_{A} \frac{\partial y}{\partial s} \frac{\partial \psi}{\partial s} d A, Q_{y z}=\int_{A} \frac{\partial x}{\partial s} \frac{\partial \psi}{\partial s} d A
\end{aligned}
$$

The corresponding boundary conditions are obtained as follows:

At the fixed end,

$$
\begin{aligned}
u=0, v & =0, w=0 \\
\theta_{x}=0, \theta_{y} & =0, \theta_{z}=0 \\
\phi & =0
\end{aligned}
$$

At the free end,

$$
\begin{aligned}
& G J_{\theta y} \theta_{z}^{\prime}-G Q_{y z} \phi+G Q_{x y}\left(v^{\prime}+\theta_{x}\right)+G Q_{y y}\left(u^{\prime}-\theta_{y}\right)=P_{x} \\
& G J_{\theta x} \theta_{z}^{\prime}-G Q_{x z} \phi+G Q_{x x}\left(v^{\prime}+\theta_{x}\right)+G Q_{x y}\left(u^{\prime}-\theta_{y}\right)=P_{y}
\end{aligned}
$$




$$
\begin{gathered}
E A w^{\prime}=P_{z} \\
-E \Gamma_{z x} \phi^{\prime}+E I_{x x} \theta_{x}^{\prime}-E I_{x y} \theta_{y}^{\prime}=M_{x} \\
E \Gamma_{z y} \phi^{\prime}+E I_{x y} \theta_{x}^{\prime}-E I_{y y} \theta_{y}^{\prime}=M_{y} \\
G J_{\theta \theta} \theta_{z}^{\prime}-G J_{\theta z} \phi+G J_{\theta x}\left(v^{\prime}+\theta_{x}\right)+G J_{\theta y}\left(u^{\prime}-\theta_{y}\right)=M_{z} \\
E \Gamma_{z z} \phi^{\prime}-E \Gamma_{z x} \theta_{x}^{\prime}-E \Gamma_{z y} \theta_{y}^{\prime}=-M_{\omega}
\end{gathered}
$$

\subsubsection{Beam Vibration}

In our study, the focus will be on a thin-walled beam of rectangular cross section. Thus, because of symmetry of this cross section, the system of coupled governing equations are reduced since some of the cross-axis constants, such as $Q_{x y}, I_{x y}, J_{\theta x}$ and $J_{\theta y}$ are all zero. The contour origin is assumed to be located on the axis of symmetry thus the warping displacement of the contour origin can also be dropped. This means that the points of contour origins along the span do not warp due to the fact that every point along the fold of symmetry is fixed. With these modifications, the reduced system of governing equations for the case of $y-y$ symmetry along the entire span can be written as follows:

$$
\begin{gathered}
-G Q_{y y}\left(u^{\prime \prime}-\theta_{y}^{\prime}\right)+\rho\left(A \ddot{u}-S_{x x} \ddot{\theta}_{z}\right)=p_{x} \\
-E \Gamma_{z y} \phi^{\prime \prime}-E I_{y y} \theta_{y}^{\prime \prime}-G Q_{y y}\left(u^{\prime}-\theta_{y}\right)+\rho\left(I_{y y} \ddot{\theta}_{y}+\Gamma_{z y} \ddot{\theta}\right)=m_{y} \\
-G J_{\theta \theta} \theta_{z}^{\prime \prime}+G J_{\theta z} \phi^{\prime}+\rho\left(\left(I_{x x}+I_{y y}\right) \ddot{\theta}_{z}-S_{x x} \ddot{u}+S_{y y} \ddot{v}\right)=m_{z} \\
-E \Gamma_{z z} \phi^{\prime \prime}-E \Gamma_{z y} \theta_{y}^{\prime \prime}-G J_{\theta z} \theta_{z}^{\prime}+G J_{z z} \phi+\rho\left(\Gamma_{z z} \ddot{\phi}+\Gamma_{z y} \ddot{\theta}_{y}\right)=-m_{\omega}
\end{gathered}
$$

These equations could be solved by using the eigenfunction expansion approach both in the spatial and time domains. Herein, it is proposed to use the assumed-mode approach wherein admissible functions that satisfy the essential boundary conditions for all the variables are used. As all the variables possess identical essential boundary conditions involving only the nonderivative functions, the independent admissible functions are used as follows [134]:

$$
\psi_{i}(z)=\frac{Z}{L}\left(1-\frac{Z}{L}\right)^{i-1} \quad \text { where } i=1,2,3, \ldots n
$$


Each degree of freedom is then expressed in terms of the admissible functions and the generalized time dependent coordinates as follows:

$$
\begin{aligned}
\phi(z, t) & =\sum_{i=1}^{n} \psi_{i}^{(1)}(z) a_{i}^{(1)}(t) \\
u(z, t) & =\sum_{i=1}^{n} \psi_{i}^{(2)}(z) a_{i}^{(2)}(t) \\
\theta_{y}(z, t) & =\sum_{i=1}^{n} \psi_{i}^{(3)}(z) a_{i}^{(3)}(t) \\
\theta_{z}(z, t) & =\sum_{i=1}^{n} \psi_{i}^{(4)}(z) a_{i}^{(4)}(t)
\end{aligned}
$$

where $a_{i}$ represents generalized coordinates associated with each degree of freedom. Although it is possible to use different admissible functions for each degree of freedom as shown in Eqs. (3.37) to (3.40), here we use the same admissible functions given in Eq. (3.36) for all degrees of freedom for obtaining the numerical results that are presented later.

The governing equations in Eq. (3.32) to (3.35) were expressed in terms of the variables of the degrees of freedom. To obtain these equations in terms of the generalized coordinates described above, the virtual work is now utilized as follows:

$$
\begin{gathered}
\delta W_{\text {Internal }}-\delta W_{\text {Inertia }}=\delta W_{\text {External }} \\
\int_{0}^{L}\left[E \Gamma_{z z} \phi^{\prime} \delta \phi^{\prime}-E \Gamma_{z y}\left(\phi^{\prime} \delta \theta_{y}^{\prime}+\theta_{y}^{\prime} \delta \phi^{\prime}\right)+E I_{y y} \theta_{y}^{\prime} \delta \theta_{y}^{\prime}+G J_{\theta \theta} \theta_{z}^{\prime} \delta \theta_{z}^{\prime}-G J_{\theta z}\left(\theta_{z}^{\prime} \delta \phi+\phi \delta \theta_{z}^{\prime}\right)\right. \\
\left.+G J_{z z} \phi \delta \phi+G Q_{y y}\left(\theta_{y}+u^{\prime}\right) \delta\left(\theta_{y}+u^{\prime}\right)\right] d z \\
-\int_{0}^{L} \rho\left[\left(I_{y y} \ddot{\theta}_{y}-\Gamma_{z y} \ddot{\phi}\right) \delta \theta_{y}+\left(\Gamma_{z z} \ddot{\phi}-\Gamma_{z y} \ddot{\theta}_{y}\right) \delta \phi+A \ddot{u} \delta u+\left(I_{x x}+I_{y y}\right) \ddot{\theta}_{z} \delta \theta_{z}\right] d z \\
=\int_{0}^{L}\left[-m_{\omega} \delta \phi+p_{x} \delta u+m_{y} \delta \theta_{y}+m_{y} \delta \theta_{z}\right] d z+\left(-M_{\omega} \delta \phi+P_{x} \delta u+M_{y} \delta \theta_{y}+M_{z} \delta \theta_{z}\right)
\end{gathered}
$$

Using Eq. (3.37) to (3.40) in Eq. (3.42) and applying the variational approach, the continuous system can be represented by the following discretized equations of motion expressed in terms of the generalized coordinates.

$$
\sum_{j=1}^{n} M_{i j} \ddot{a}_{j}+\sum_{j=1}^{n} K_{i j} a_{j}=F_{i}
$$


where

$$
M_{i j}=\int_{0}^{L} \rho\left[\left(I_{y y} \psi_{i}^{(3)}-\Gamma_{z y} \psi_{i}^{(1)}\right) \psi_{j}^{(3)}+\left(\Gamma_{z z} \psi_{i}^{(1)}-\Gamma_{z y} \psi_{i}^{(3)}\right) \psi_{j}^{(1)}+A \psi_{i}^{(2)} \psi_{j}^{(2)}+\left(I_{x x}+\right.\right.
$$

$\left.\left.I_{y y}\right) \psi_{i}^{(4)} \psi_{j}^{(4)}\right] d z$

$$
K_{i j}=\int_{0}^{L}\left[E \Gamma_{z z} \psi_{i}^{(1)^{\prime}} \psi_{j}^{(1)^{\prime}}-E \Gamma_{z y}\left(\psi_{i}^{(1)^{\prime}} \psi_{j}^{(3)^{\prime}}+\psi_{i}^{(3)^{\prime}} \psi_{j}^{(1)^{\prime}}\right)+E I_{y y} \psi_{i}^{(3)^{\prime}} \psi_{j}^{(3)^{\prime}}+\right.
$$

$G J_{\theta \theta} \psi_{i}^{(4)^{\prime}} \psi_{j}^{(4)^{\prime}}-G J_{\theta z}\left(\psi_{j}^{(4)^{\prime}} \psi_{j}^{(1)}+\psi_{i}^{(1)} \psi_{j}^{(4)^{\prime}}\right)+G J_{z z} \psi_{i}^{(1)} \psi_{j}^{(1)}+G Q_{y y}\left(\psi_{i}^{(3)}+\psi_{i}^{(2)^{\prime}}\right)\left(\psi_{j}^{(3)}+\right.$ $\left.\left.\psi_{j}^{(2)^{\prime}}\right)\right] d z$

$$
F_{i}=\int_{0}^{L}\left[-m_{\omega} \psi_{i}^{(1)}+p_{x} \psi_{i}^{(2)}+m_{y} \psi_{i}^{(3)}+m_{y} \psi_{i}^{(4)}\right] d z+\left(-M_{\omega} \psi_{i}^{(1)}+P_{x} \psi_{i}^{(2)}+M_{y} \psi_{i}^{(3)}+\right.
$$

$\left.M_{z} \psi_{i}^{(4)}\right)$

The system of equations in Eq. (3.32) to (3.35) is rewritten using matrix expression as follows:

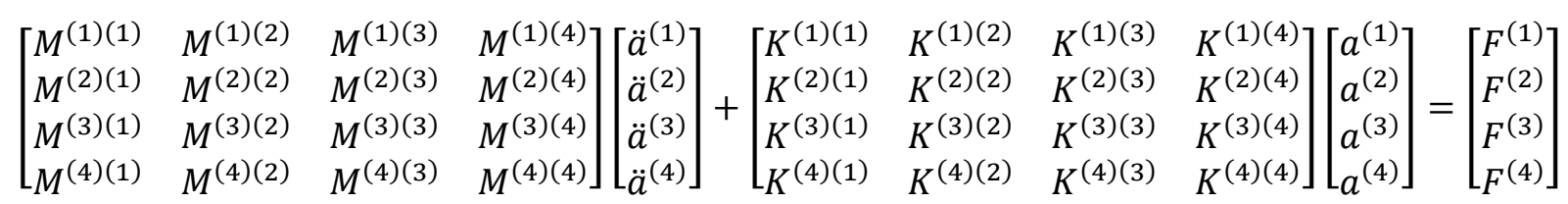

where the sub-matrices $M^{(l)(m)}$ and $K^{(l)(m)}$ each are $n \times n$, the elements of which are $M_{i j}^{(l)(m)}$ and $M_{i j}^{(l)(m)}$. The sub-vectors of the generalized coordinates, $a^{(m)}$, are of size $n \times 1$, the elements of which are $a_{i}^{(m)}(t)$; the sub-vector of the generalized forces, $F^{(l)}$, are of size $n x 1$, the elements of which are $F_{i}^{(l)}$.

Eq. (3.44) is rewritten in the compact matrix form as follows:

$$
\llbracket M \rrbracket\{\ddot{a}\}+\llbracket K \rrbracket\{a\}=\{F\}
$$

where $\llbracket M \rrbracket$ and $\llbracket K \rrbracket$ are of size $4 n \times 4 n$ and the vectors of the generalized coordinates $\{a\}$ and forcing vectors $\{F\}$ are of size $4 n \times 1$. This equation can be solved using traditional methods without any difficulties. The free vibration analysis of this equation provides the system 
frequencies $\omega_{i}$ and corresponding modes (eigenvectors), $\vec{u}_{i}$. These modes and natural frequencies can be used in the standard modal analysis approach to obtain the generalized coordinates and thus the response. For the applied forces defined as random processes, it is convenient to express the response in the frequency domain as explained in the next section.

Table 3.1 presents the comparison of the frequencies of the thin walled beam calculated by the Benscoter theory (the present formulation), Vlasov beam theory, and those calculated by the shell finite element analysis. These results are obtained for a thin walled beam of the dimensions: beam width $=0.4 \mathrm{~m}$, beam height $=0.2 \mathrm{~m}$, wall thickness $=0.01 \mathrm{~m}$, the span length $=6 \mathrm{~m}$. The details of the shell finite element analysis used here in this comparison are given in Appendix B. A first few frequencies associated with bending modes are estimated accurately by the thin walled beam theories. The torsion mode frequency is, however, overestimated by about $8 \%$. This is simply because the shell finite element allows the out-of-plane element deformations whereas the thin walled beam theories assume the cross section to be rigid thus not permitting any wall deformation out of the beam surface.

Table 3.1 Comparison of natural frequencies $(\mathrm{Hz})$ of a rectangular thin-walled cantilever

\begin{tabular}{cccc}
\hline Mode & $\begin{array}{c}\text { Vlasov } \\
\text { beam }\end{array}$ & $\begin{array}{c}\text { This } \\
\text { formulation }\end{array}$ & Shell \\
\hline $1^{\text {st }}$ Major-axis Bending & 7.07 & 7.04 & 7.00 \\
$1^{\text {st }}$ Minor-axis Bending & 11.9 & 11.9 & 11.8 \\
$2^{\text {nd }}$ Major-axis Bending & 44.3 & 43.0 & 42.2 \\
$2^{\text {nd }}$ Minor-axis Bending & 74.9 & 71.4 & 71.1 \\
$1^{\text {st }}$ Twisting & 102 & 102 & 94.7 \\
$3^{\text {rd }}$ Major-axis Bending & 124 & 116 & 109 \\
\hline \hline
\end{tabular}

\subsubsection{Random Responses of Beam}

For the applied load defined as a random process, the beam response is also a random process. The random characteristics of such loads are often defined by their spectral and cross spectral density function. For such description, it is usually convenient to work in the frequency domain 
to obtain the beam response. It can be shown that the cross spectral density function of any two displacement, rotation or warping response quantities can be expressed as follows:

$$
\Phi\left(z_{1}, z_{2} ; \omega\right)=\sum_{j=1}^{n} \sum_{k=1}^{n} f_{j}\left(z_{1}\right) f_{k}\left(z_{2}\right) H_{j}(\omega) H_{k}^{*}(\omega) F_{j k}(\omega)
$$

where $\Phi\left(z_{1}, z_{2} ; \omega\right)$ is cross spectral density function of response. $f(z)$ is the eigenfunction for the response quantity of interest. For example, for the quantities of interest here, these mode shapes are defined as follows:

$$
\begin{gathered}
f_{\emptyset_{j}}(z)=\sum_{i=1}^{n} \bar{u}_{j, i} \psi_{i}(z) \\
f_{u_{j}}(z)=\sum_{i=1}^{n} \bar{u}_{j, n+i} \psi_{i}(z) \\
f_{\theta_{y_{j}}}(z)=\sum_{i=1}^{n} \bar{u}_{j, 2 n+i} \psi_{i}(z) \\
f_{\theta_{z_{j}}}(z)=\sum_{i=1}^{n} \bar{u}_{j, 3 n+i} \psi_{i}(z)
\end{gathered}
$$

where $i=1$ to $n$ and $j=1$ to $4 n, \bar{u}$ - eigenvector matrix. $H_{i}(\omega)$ is the modal frequency response function defined [88] as follows:

$$
H_{i}(\omega)=\frac{1}{\omega_{i}^{2}-\omega^{2}-2 \zeta_{i} \omega_{i} \omega \hat{\imath}} \quad ; \quad i=1,2,3, \ldots
$$

where $\omega_{i}, \zeta_{i}$ are the natural frequency and viscous damping ratio of the $i^{\text {th }}$ mode. Herein, the viscous damping ratio is introduced to account for the inevitable energy dissipation in the system. For numerical results, a value of $1 \%$ is assumed in the beam-model as a representative value for the vibration of an aircraft wing [85].

The spectral density of the generalized forces for the modes $j$ and $k$ in Eq. (3.46) is defined [135] as follows:

$$
F_{j k}(\omega)=\int_{D} \int_{D} \Phi_{F F}\left(z_{1}^{\prime}, z_{2}^{\prime} ; \omega\right) f_{j}\left(z_{1}^{\prime}\right) f_{k}\left(z_{2}^{\prime}\right) d z_{1}^{\prime} d z_{2}^{\prime}
$$


where $\Phi_{F F}\left(z_{1}^{\prime}, z_{2}^{\prime} ; \omega\right)$ is the cross spectral density function of the external forces applied at locations $z_{1}^{\prime}$ and $z_{2}^{\prime}$ on the beam, $f_{j}\left(z_{1}^{\prime}\right)$ is the eigenfunction as indicate earlier and the integrations are over the entire domain $D$ subjected to loadings. The final expression for the spectral density function of the response quantities can be written in the following matrix form.

$$
\llbracket \Phi(z, \omega) \rrbracket=[f(z)] \llbracket H(\omega) \rrbracket\left[f\left(z^{\prime}\right)\right] \llbracket \Phi_{F F}\left(z^{\prime}, \omega\right) \rrbracket\left[f\left(z^{\prime}\right)\right] \llbracket H^{*}(\omega) \rrbracket[f(z)]
$$

where $z^{\prime}$ denotes the location of the applied load and $z$ denotes the location of the observed response.

Power spectral density function of shear stress can be defined in terms of the power spectral density function of the response variables that define the shear stress. The definition of the shear stress is as follows:

$$
\sigma_{s z}=G\left[R_{n} \theta_{z}^{\prime}-\frac{\partial \psi}{\partial s} \phi\right]
$$

The expression for the spectral density function of the shear stress is defined by

$$
\Phi_{\sigma_{s z} \sigma_{s z}}(\omega)=G^{2}\left[R_{n}^{2} \Phi_{\theta_{z}^{\prime} \theta_{Z}^{\prime}}(\omega)-R_{n} \frac{\partial \psi}{\partial s}\left(\Phi_{\theta_{z}^{\prime} \emptyset}(\omega)+\Phi_{\emptyset \theta_{z}^{\prime}}(\omega)\right)+\left(\frac{\partial \psi}{\partial s}\right)^{2} \Phi_{\varnothing \emptyset}(\omega)\right]
$$

For calculating the numerical results in this study, the applied load on the beam is defined by the von Karman's gust load power spectral density function. However, due to the fact that the structural model in this study is scaled down and occupies the range frequencies that are much higher than those of a full-scale aircraft, the power spectral density used here is modified such that it maintains the original shape but adjusts the range of frequencies. As the gust load is assumed to be distributed uniformly, the power spectral density of distributed twisting moment along the span is defined as follows: 


$$
\Phi_{F F}(\omega)=C\left[\frac{1+\frac{8}{3}(1.339 S \omega)^{2}}{\left[1+\frac{8}{3}(1.339 S \omega)^{2}\right]^{\frac{11}{6}}}\right]^{2}
$$

where $C$ is an assumed constant and $S$ is a scaled factor of frequency. It was assumed that $C=300$ and $S=0.001$. The assumed power spectral density of input is shown in Figure 3.3.

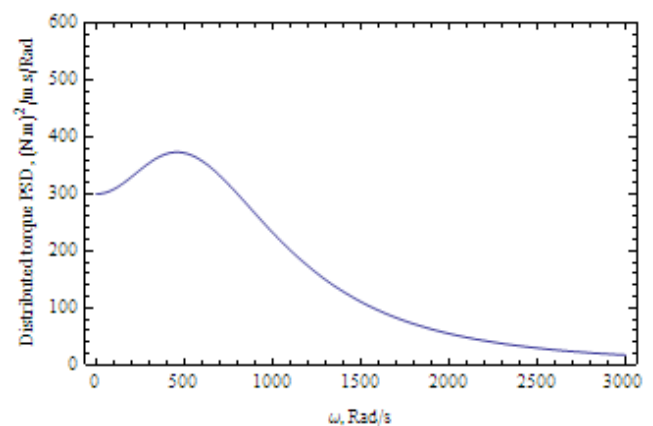

\section{Figure 3.3 Power spectral density of the twisting distributed moment (Input)}

For this random input, the power spectral density function of the shear stress response at the mid span is shown in Figure 3.4. It is observed that the higher modes falling outside the frequency range of the input spectral density function are minimized and almost do not contribute to the response.

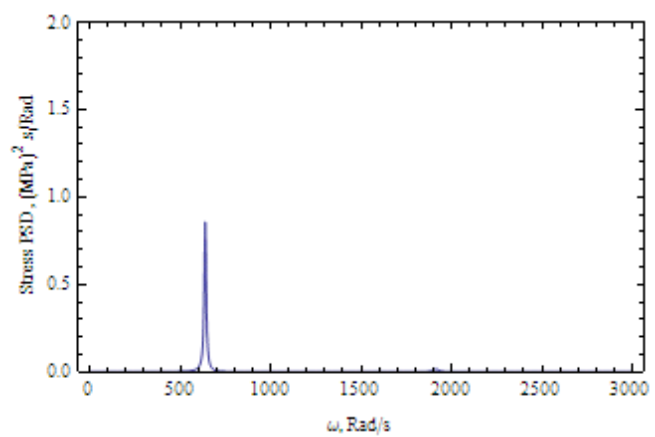

Figure 3.4 Power spectral density of the shear stress at the mid span (Output) 


\subsubsection{Stochastic Characteristics of Responses}

For crack growth analysis under dynamic random loads, the statistics of the peak stress response are necessary. Assuming that the input and the response are zero-mean Gaussian random processes, the probability density function of the peak stress is defined as follows [136]:

$$
f_{p}(p)=\frac{\epsilon}{\sqrt{\lambda_{0}}} \phi\left(\frac{p}{\epsilon \sqrt{\lambda_{0}}}\right)+\sqrt{1-\epsilon^{2}} \frac{p}{\lambda_{0}} e^{-\frac{p^{2}}{2 \lambda_{0}}} \Phi\left(\frac{p \sqrt{1-\epsilon^{2}}}{\epsilon \sqrt{\lambda_{0}}}\right)
$$

where $\phi$ and $\Phi$ are the probability density function and cumulative distribution function of the standard normal distribution, respectively; $\lambda_{k}$ are the spectral moments, which can be obtained from response power spectral density as follows:

$$
\lambda_{k}=\int_{0}^{\infty} \omega^{\mathrm{k}} \Phi_{\sigma_{s z} \sigma_{s z}}(\omega) d \omega \quad \text { where } k=1,2,3, \ldots
$$

The factor, $\alpha$, is often called the regularity factor and the spectral band width parameter, $\epsilon$, are defined in the term of the spectral moments as follows:

$$
\begin{aligned}
& \alpha_{j}=\frac{\lambda_{j}}{\sqrt{\lambda_{0} \lambda_{2 j}}} \\
& \epsilon=\sqrt{1-\alpha^{2}} \quad \text { where } \alpha=\alpha_{2}
\end{aligned}
$$

For a narrow band process, for which $\epsilon$ is small (nearly zero) the distribution in Eq. (3.57) approaches a Raleigh distribution. For the broad band case when this parameter is nearly one, the distribution approaches the Gaussian distribution as follows:

$$
\begin{array}{cc}
f_{p}(p)=\frac{p}{\lambda_{0}} e^{-\frac{p^{2}}{2 \lambda_{0}}} & \text { if } \epsilon \approx 0 \\
f_{p}(p)=\frac{1}{\sqrt{\lambda_{0}}} \phi\left(\frac{p}{\sqrt{\lambda_{0}}}\right) & \text { if } \epsilon \approx 1
\end{array}
$$

The plot in Figure 3.5 compares the probability density function of stress peak obtained at mid span (L/2) for the example problem considered here with the corresponding narrow and 
broad band density functions. It should be noted that the peak stress distribution is nearly a Raleigh distribution.

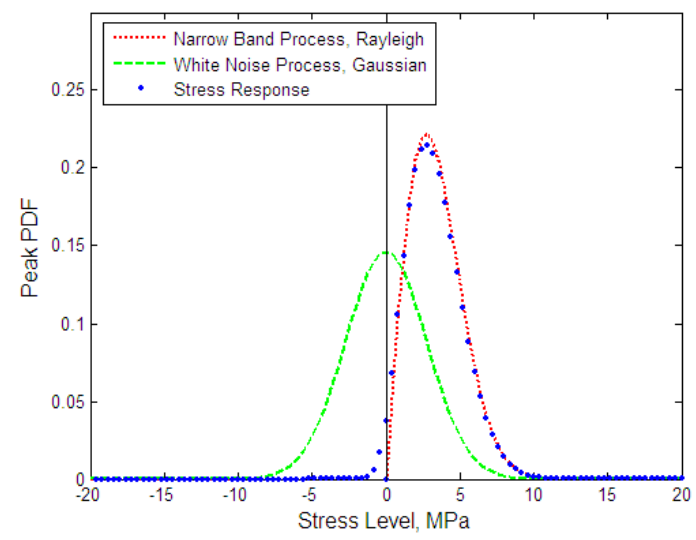

Figure 3.5 Comparison of the calculated probability density function of the peaks of the shear stress at mid span with the Raleigh and Gaussian density functions

For the example problem of a beam, Table 3.2 shows the three spectral moments and the parameters $\alpha$ and $\epsilon$ calculated at three beam locations.

Table 3.2 Statistical parameters of shear stresses at different locations

\begin{tabular}{cccccc}
\hline \hline $\begin{array}{c}\text { Location from the fixed end } \\
\text { (Span length=L) }\end{array}$ & $\begin{array}{c}\lambda_{0} \\
(\text { MPa })^{2}\end{array}$ & $\begin{array}{c}\lambda_{2} \\
(\text { MPa.rad/s })^{2}\end{array}$ & $\begin{array}{c}\lambda_{4} \\
\left(\text { MPa. }(\mathrm{rad} / \mathrm{s})^{2}\right)^{2}\end{array}$ & $\alpha$ & $\epsilon$ \\
\hline $\mathrm{L} / 4$ & 20.31 & $8.277 \times 10^{6}$ & $3.389 \times 10^{13}$ & 0.998 & 0.070 \\
$\mathrm{~L} / 2$ & 7.434 & $3.052 \times 10^{6}$ & $1.341 \times 10^{12}$ & 0.967 & 0.256 \\
$\mathrm{SL} / 4$ & 0.692 & $3.568 \times 10^{5}$ & $4.390 \times 10^{11}$ & 0.647 & 0.762 \\
\hline \hline
\end{tabular}

Figure 3.6 shows the mean square value of the shear stress obtained at these three locations. In a static case, this shear stress caused by a uniform twisting moment due to gust loads is linearly increasing from the tip to the fixed end. In the dynamic case, similarly, the inertial effect being significant cause higher levels of stress near the fixed support. Thus, a crack near the fixed support is likely to grow faster. However, the dynamic stress does not vary linearly as the static case. 


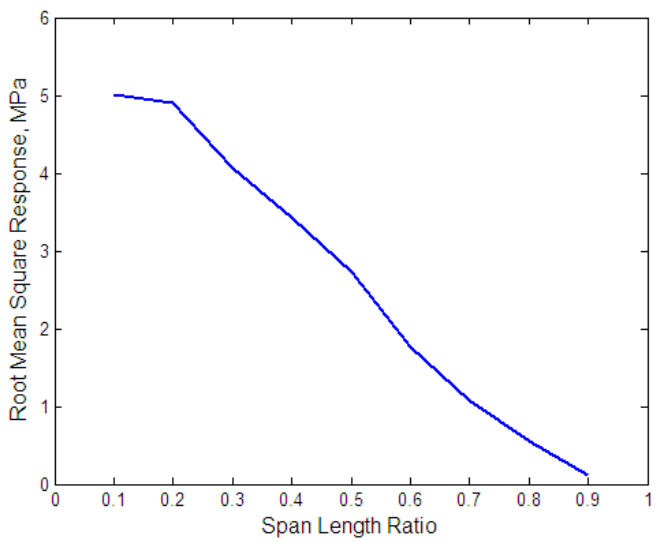

Figure 3.6 Root mean square values of dynamic shear stresses at various locations along the span

\subsection{Crack Growth Simulations under Stochastic Loadings}

To use the crack growth rate equation, one needs to know the frequency of loading $f_{s}$, the stress range, $\Delta \sigma$, and stress ratio factor $R$ for the applied load. For a constant amplitude cyclic loading for which the crack growth rate models were originally intended to be used, these parameters could be easily defined. For a random train of cycles, however, it is not quite clear how to define these quantities. To calculate these quantities for a random response, several different interpretations have been proposed. For example, to calculate the stress range, Sobczyk [99] suggested to use twice the value of the root mean square response. Later, however, it was suggested to use the peak response characteristics of the stress to define the quantities of the average minimum stress, the average maximum stress, the stress range and the stress ratio as follows:

$$
\begin{gathered}
\sigma_{\text {min }}=\sigma_{\text {mean }}-E[p] \\
\sigma_{\text {max }}=\sigma_{\text {mean }}+E[p] \\
\Delta \sigma=2 E[p] \\
R=\frac{\sigma_{\text {min }}}{\sigma_{\text {max }}}=\frac{\sigma_{\text {mean }}-E[p]}{\sigma_{\text {mean }}+E[p]}
\end{gathered}
$$

where $\sigma_{\text {mean }}$ is the average static level of the stress and $E[p]$ is the average peak stress which can be obtained from the probability function of the peak stress as follows: 


$$
E[p]=\int_{-\infty}^{\infty} p f_{p}(p) d z
$$

The probability density function of the peak stress is defined by Eq. (3.57). Substituting the expression of the density function, the average peak stress can be calculated as follows:

$$
E[p]=\sigma_{r m s} \sqrt{\frac{\pi}{2}\left(1-\epsilon^{2}\right)}
$$

where $\sigma_{r m s}$ is the square root of the zeroth order spectral moment $\left(\lambda_{0}\right)$. In the numerical example, the average static stress value $\sigma_{\text {mean }}$ is assumed to be $60 \mathrm{MPa}$.

Another parameter that needs to be defined for calculation of the crack growth rate is the equivalent frequency value, $f_{s}$. In a random sequence of stress cycles there is no one frequency that can be used, so a decision has to be made. Herein the stress response is strongly narrow band as indicated by a very small value of the parameter, $\epsilon$. In this case, thus, it is appropriate to use in place of equivalent $f_{s}$, the zero crossing rate defined by the following equation,

$$
f_{S} \cong \frac{1}{2 \pi} \sqrt{\frac{\lambda_{2}}{\lambda_{0}}}
$$

In Table 3.3, some of these parameters can be calculated for the example problem. These values are used in the crack growth simulation described in the following section.

Table 3.3 Equivalent deterministic parameters of stress responses at different locations

\begin{tabular}{cccccc}
\hline $\begin{array}{c}\text { Distance from the fixed end } \\
(\text { Span=L })\end{array}$ & $\begin{array}{c}\sigma_{r m s} \\
(M P a)\end{array}$ & $\begin{array}{c}\Delta \sigma \\
(M P a)\end{array}$ & $\begin{array}{c}\sigma_{\min } \\
(M P a)\end{array}$ & $\begin{array}{c}\sigma_{\max } \\
(M P a)\end{array}$ & $R$ \\
\hline $\mathrm{L} / 4$ & 4.51 & 11.27 & 54.37 & 65.63 & 0.828 \\
$\mathrm{~L} / 2$ & 2.73 & 6.61 & 56.70 & 63.30 & 0.896 \\
$3 \mathrm{~L} / 4$ & 0.83 & 1.35 & 59.33 & 60.68 & 0.978 \\
\hline
\end{tabular}


To perform the crack growth analysis, it is assumed that the overall response of the beam stress remains unchanged as the crack grows, so that uncracked beam response can still be used to examine the growth with proper use of the stress intensity factor. For the numerical simulation, it is assumed that initial crack size is a Gaussian random variable with mean and standard deviation values of $N(150,10) \mathrm{mm}$. The geometric factor of stress intensity factor is defined by Eq. (3.5). The fracture toughness is assumed to be $N(2600,130) M P a \cdot \sqrt{m m}[137]$; $C, m$ and $q$ are aluminum constants used in the crack growth model obtained from NASGRO [126] where $C=0.2382 \times 10^{-11}, m=3.2$ and $q=1$.

The simulation is based on Monte Carlo approach and proceeds as follows. To calculate the stress intensity factor, random sample values of the crack size are generated. The distribution of the critical crack length is defined using Eq. (3.2). For the applied stochastic load, the stress range and the stress ratio needed in Eq. (3.1) are then calculated according to Eq. (3.65). Equation (3.1) is then integrated from one time step to another. At each time step, a random value of $X(t)$ is generated to introduce the intermingling of the growth curves.

The simulation results obtained for three different locations of the crack are shown in Figure 3.7. Each growth curve corresponds to a set of sample values for which Eq. (3.1) was integrated. From these simulation results, it is observed that a crack near the fixed support can very quickly grow to approach the critical crack length that causes failure. With increasing time duration, it can also be observed that a large dispersion shows up in the simulation results. This is clearly shown in the set of the results shown in Figure 3.8 where the plot of the histogram of the crack sizes is depicted at different time for the three crack locations. As time increases, larger and larger numbers of sample values are seen to approach the critical length indicating the failure. This fraction of samples that reach the critical length out of the ensemble of the total samples considered in the simulation gives the estimate of the probability of failure at that time. Also this dispersion is larger near the fixed end where the presence of a crack is certainly more critical. In these figures, the log-normal distributions are plotted correspondingly with the same mean and standard deviations as the sample values in the histogram. For larger time steps, the actual distribution becomes highly skewed and thus cannot be reasonably represented by a log-normal distribution. 
The analytically defined probability distributions at different times, described, could not be used to calculate the probability of failure. The failure is defined to occur when the crack size exceeds the critical crack size; defined by Eq. (3.2). The probability distribution of this crack size is obtained by simulation. Figure 3.8 shows the sample histograms obtained at the three locations while Figure 3.9 depicts the corresponding distributions of critical crack size in the form of a histogram fitted with associated log-normal curves. Although the two figures, Figures 3.8 and 3.9, can be used to evaluate the probability of failure by the approach of standard stress-strength interference, it is more convenient to evaluate this value by counting the number of failure samples with respect to the time using Figure 3.7.

Figure 3.10 shows the growth in the probability of failure with time at the three crack locations on the beam. The probability of failure increases at a much faster rate for a crack near the support. For the example problem considered here, the probability of failure for a crack near the tip, on the other hand, is very low. In fact, in the simulation conducted in this study no sample is seen to reach the critical length for crack near the tip. For a probability of exceedance value of $25 \%$, a cracked panel at the location of L/4 fails at 1.2 hours and the panel at mid span, $\mathrm{L} / 2$ fails at 6.8 hours. The cracked panel near the tip at the location 3L/4, however, does not fail within the investigated period of seven hours as the growth of crack at this location is rather slow but the rapid growth of crack size near the support where the stress level is high, and the fact that inertial effects also increase stress response to be higher near the fixed support, clearly show that the crack growth depends upon the level of stress and the dynamic effect.
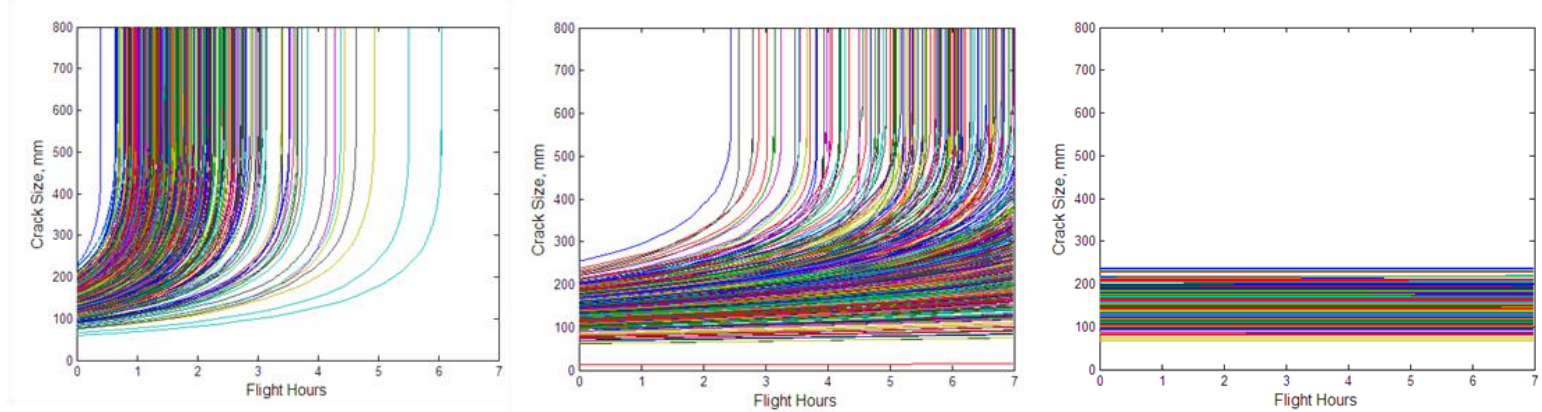

Figure 3.7 Evolution of crack growth with time for three damage locations, $L / 4$ (left), $L / 2$ (middle) and $3 L / 4$ (right) from the fixed end 

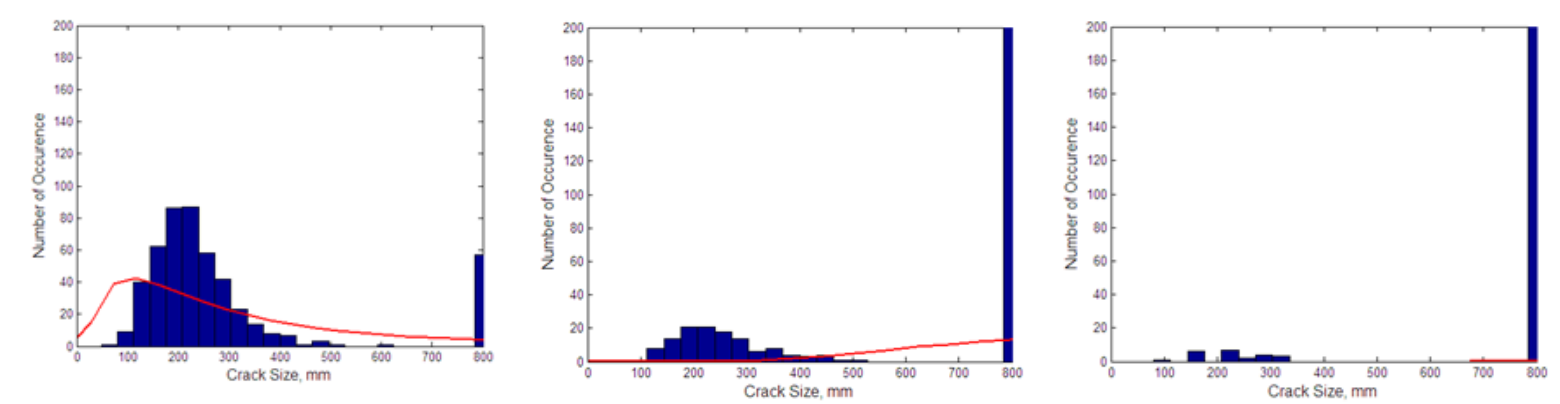

a) $L / 4$
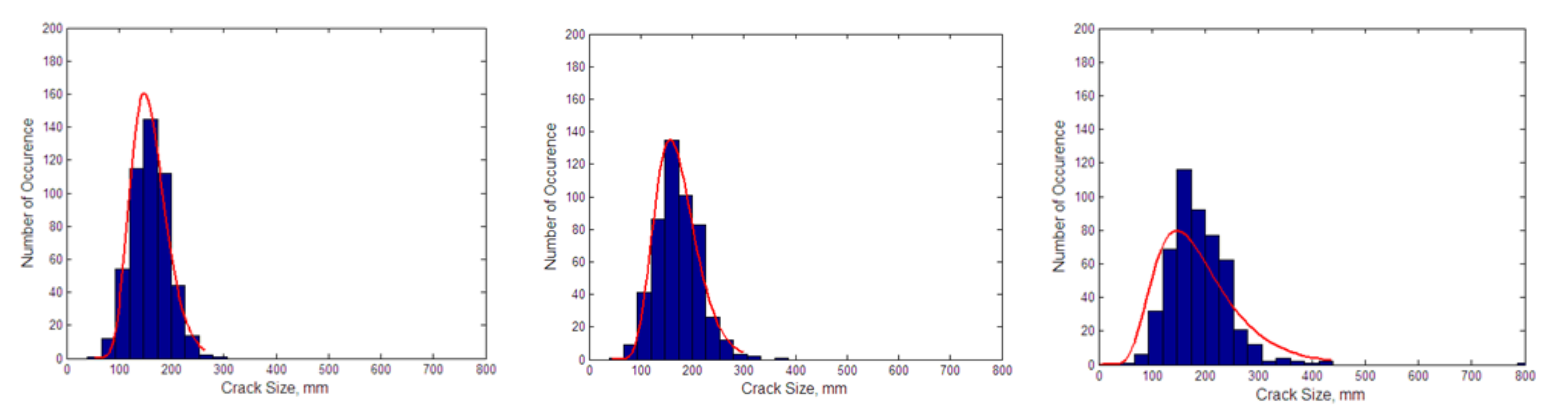

b) $\mathrm{L} / 2$
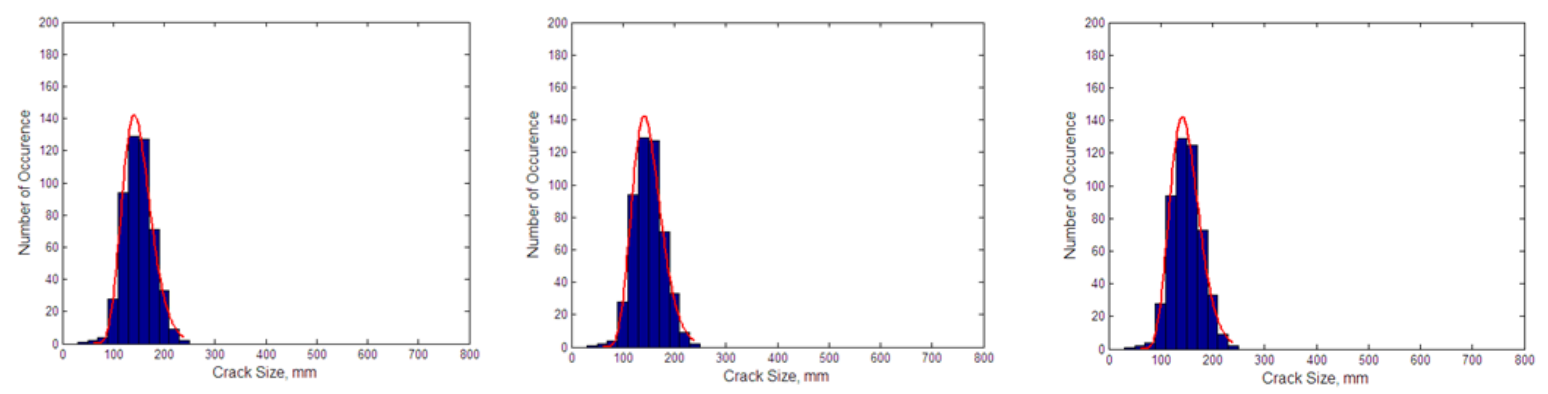

c) $3 \mathrm{~L} / 4$

Figure 3.8 Histograms of the crack length distribution at three time instants of 1 hour (left), 2 hours (middle) and 3 hours (right) for three damage locations from the fixed end
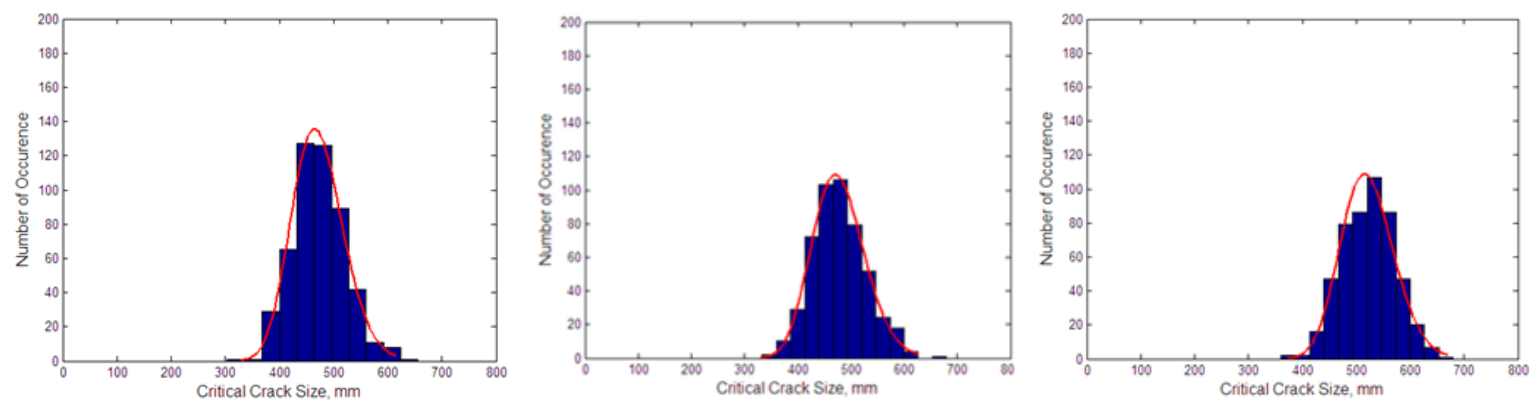

Figure 3.9 Critical crack length distribution for three damage locations, $L / 4$ (left), $L / 2$ (middle) and $3 L / 4$ (right) from the fixed end 

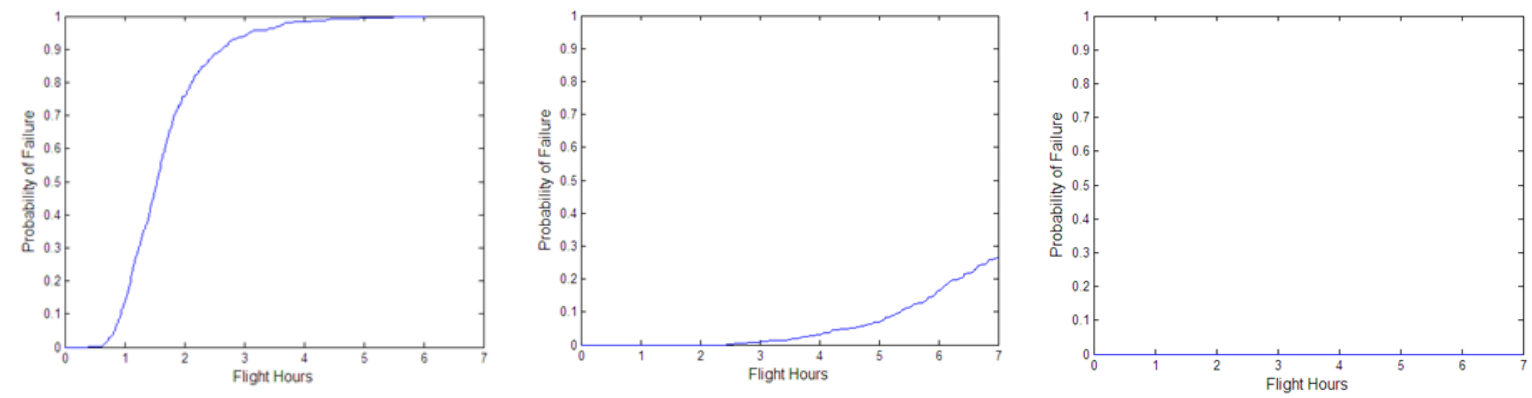

Figure 3.10 Time evolution of the probability of failure for three damage locations, $L / 4$ (left), $L / 2$ (middle) and $3 L / 4$ (right) from the fixed end

\subsection{Summary and Concluding Remarks}

This paper describes a methodology for probabilistic growth analysis of a damaged thin walled beam subjected to stochastically defined loads with the ultimate objective of using it on an aircraft wing. It is assumed that the state of stress in the uncracked beam with proper stress concentration factor can be used in predicting the crack growth rate. The paper, thus, describes the analysis of a thin walled beam subjected to a set of random loads. First, a general formulation which can be used with general loading conditions is developed. The numerical results are, however, obtained for a beam subjected to a uniform twisting moment induced by gust loads. The coupled equations of motion are discretized by using the assumed-mode approach and the statistics of the response needed in crack growth simulation are obtained by frequency domain analysis. The Forman equation is used to model the crack growth. The growth rate simulation results are obtained by solving the differential equation numerically for a large sample of randomly generated values of the variables in the model. The peak response statistics is calculated by random vibration analysis of the beam. The results of this simulation are then used in calculating the probability of failure, providing the probability of failure with time. The results are obtained for three crack locations on the beam. These results clearly show that a crack near the fixed end grows most rapidly because the shear stress is higher near the fixed end as well as because of the enhanced dynamic effects of the beam vibrations near the fixed end. 
Chapter 4: Aeroelastic Divergence of a Thinwalled Beam with a Crack 
Aeroelastic Divergence of a Thin-walled Beam with a Crack Chalitphan Kunaporn, Mahendra P. Singh, Mayuresh J. Patil and Rakesh K. Kapania Virginia Polytechnic Institute and State University, Blacksburg, VA 24061

Aeroelastic divergence which strongly depends on the torsional stiffness of the aircraft wings, is of concern in the design of aircraft. This paper examines the effect of a discrete damage such as a crack on the divergence dynamic pressure of a wing. The wing is represented by a simple model of a thin-walled beam with a crack with random characteristics. Two approaches are used to calculate the divergence dynamic pressure: an iterative approach when the mathematical model is difficult to analyze and the direct approach which can only be used if the response for the model can be expressed in closed form. For the assumed simple model of a thin-walled beam with a longitudinal crack both approach could be applied. The effects of the location and the size of the crack on the divergence dynamic pressure are examined. The effect of the uncertainties in the crack location and size on the divergence dynamic pressure is examined probabilistically.

\subsection{Introduction}

In the design of aircraft wings, aeroelastic divergence is a significant constraint that needs to be satisfied. Divergence is a structural instability in which, depending upon the wing's torsional rigidity, the twisting moments induced by aerodynamic forces [138, 139] and corresponding torsional deformations can grow large with undesirable consequences. The twisting moment, the angle of twist and the angle of attack are three inter-dependent quantities. The angle of attack is equal to the sum of initial angle of attack and the angle of twist. Since the angle of attack affects the twisting moment which in turn determines the angle of twist, these quantities feed into each other. Depending upon the torsional rigidity and the airfoil characteristics, this feedback may create a situation with ever increasing twisting moment and the torsional response of the wing leading to an undesirable response. Since this phenomenon is highly dependent on the wing's torsional rigidity and since a discrete source damage lowers the wing's rigidity, it is important to

\footnotetext{
${ }^{*}$ This article is prepared for submission to the journal of aircraft.
} 
examine the effect of such damage on this response. In this paper, we, thus, focus on the study of the aeroelastic divergence of a wing with a crack as it will not only affect the strength of structure but also the stiffness, hence its aeroelasticity behavior.

This study utilizes a simple model of a hollow thin-walled beam with a longitudinal crack representing the damaged wing. The portion of the beam with the crack is modeled as an open section thin-walled beam, whereas the other two sections are represented by closed section thinwalled beams. The analysis of such a thin-walled beam with discontinuities has received only limited attention from the researchers. After a detailed literature survey, it was found that the approach proposed by Gunnlaugsson and Pedersen published in a series of papers [18, 20, 130] perhaps provides the most accurate treatment to the problem. Their formulation is based on the Benscoter's thin-walled beam element [8]. To include the effect of an opening in the finite element analysis of a thin-walled beam, Gunnlaugsson and Pedersen proposed to use special compatibility conditions at the interfaces of the open and closed cross-sections. In our study, the Gunnlaugsson and Pedersen compatibility conditions are used to formulate an analytical approach to solve the problem of a thin-walled beam with a crack.

The initial part of the paper develops the governing equations of equilibrium of a thin-walled beam with a crack along with the necessary compatibility and boundary conditions. These equations are coupled. In their original complicated form they cannot be solved individually. To allow them to be solved conveniently, these equations are re-stated in state-space form which permits them to be decoupled using the Jordan canonical form approach. These uncoupled equations can be solved to obtain the response in an exact sense for any arbitrary loading on the beam. The validity that the Benscoter's thin-walled beam theory [8] coupled with Gunnlaugsson and Pedersen compatibility conditions can provide accurate response values is numerically established through detailed shell finite element analysis of an example problem.

The above approach is used to express the angle of twist as a function of the aerodynamic pressure loading analytically. The divergence condition with this analytical solution is used to calculate the minimum pressure at which divergence in the response occurs. The validity of this approach is verified by comparing the results obtained with the traditional divergence solution 
approach. Finally, this approach is used to calculate the divergence dynamic pressure for different characteristics of the crack, such as its location along the beam and its size. Since the size and location parameters of a crack are random quantities, a probabilistic measure of divergence possibility is required. For this, the proposed analytical approach is also used to probabilistically characterize the divergence dynamic pressure for randomly defined crack size and location.

\subsection{Aeroelastic Modeling of a Wing as a Cracked Thin-walled Beam}

A low-order, computationally-efficient aeroelastic model of a wing is required to study the effect of uncertainties in discrete source damage on divergence instability. We consider a hollow thin-walled beam of rectangular cross section with a longitudinal crack on one of its faces subjected to aerodynamics loads. This wing model is shown in Figure 4.1. The damage parameters considered are the crack length and its location along the beam and they are assumed to be uncertain quantities with known mean and standard deviations. The beam is divided into three parts with the cracked part between the two undamaged parts. The undamaged beam portions are represented as the closed cross-section beam and the damaged portion is represented as the open cross-section beam as shown in Figure 4.1. The kinematics of thin-walled beam are described using the combination of Cartesian coordinate $(x, y, z)$ and orthogonal curvilinear coordinate $(s, n, z)$. For convenience, the global $(Z)$ and local $\left(z_{i}\right)$ coordinates are used interchangeably.

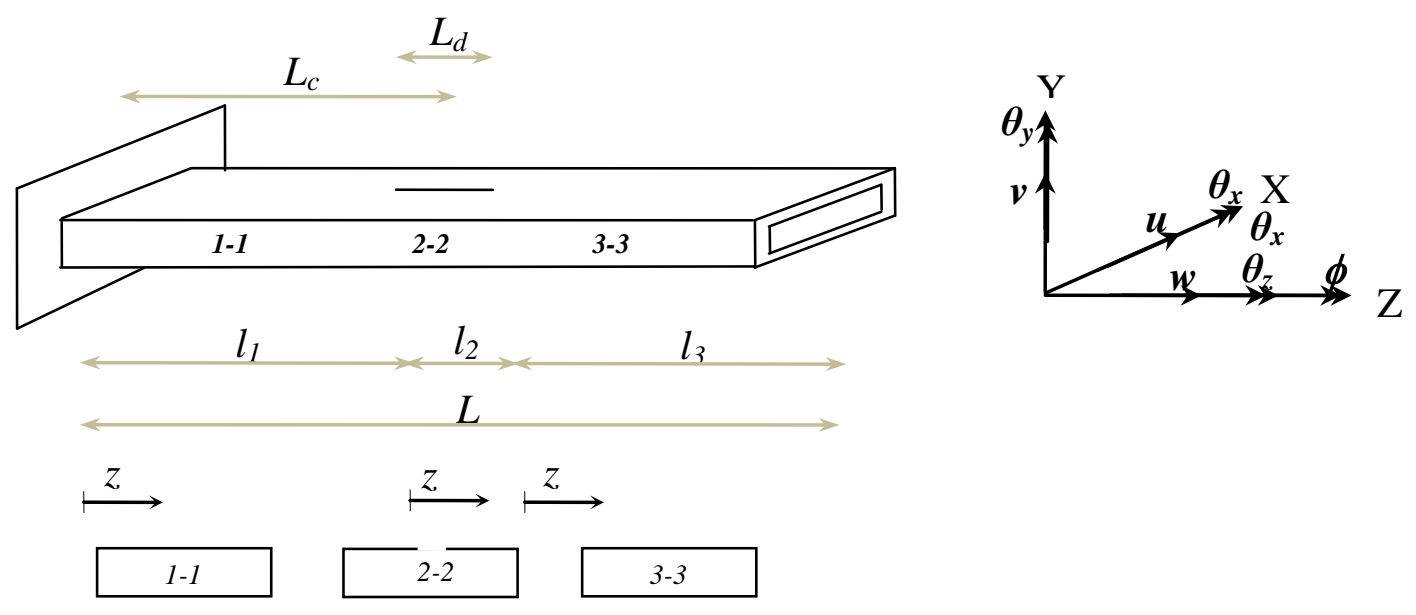

Figure 4.1 Damaged structure modeled with a cracked thin-walled cantilever 
The closed cross-section is assumed to be not deformable in its own plane. Therefore, only rigid body motions are allowed for the in-plane displacements. The warping in both cross sections introduces axial deformations along the $z$-direction and shear deformations in the plane of the cross section. An arbitrary point, $P\left(x_{p}, y_{p}\right)$, or so-called "Pole" is used as the reference to describe all displacement fields. The poles of cross-sections along the beam span are chosen at the shear centers and the angle of twist, $\theta_{z}$, is represented relative to this point. Only two coordinates, $\theta_{z}$, and warping displacement, $w$, are needed to define the displacement and stress field to derive the governing equations. The tangential displacements, $v_{s}$, and the out-of-plane warping displacement, $w$ are used to define the displacement field in the $(s, n, z)$ coordinate system. To calculate the warping function of a thin-walled section, we choose a reference point, $C O\left(x_{c o}, y_{c o}\right)$, where the coordinate $s=0$ for contour integration. The dimensions $b$ and $h$, respectively, are the width and height of the beam cross-section.

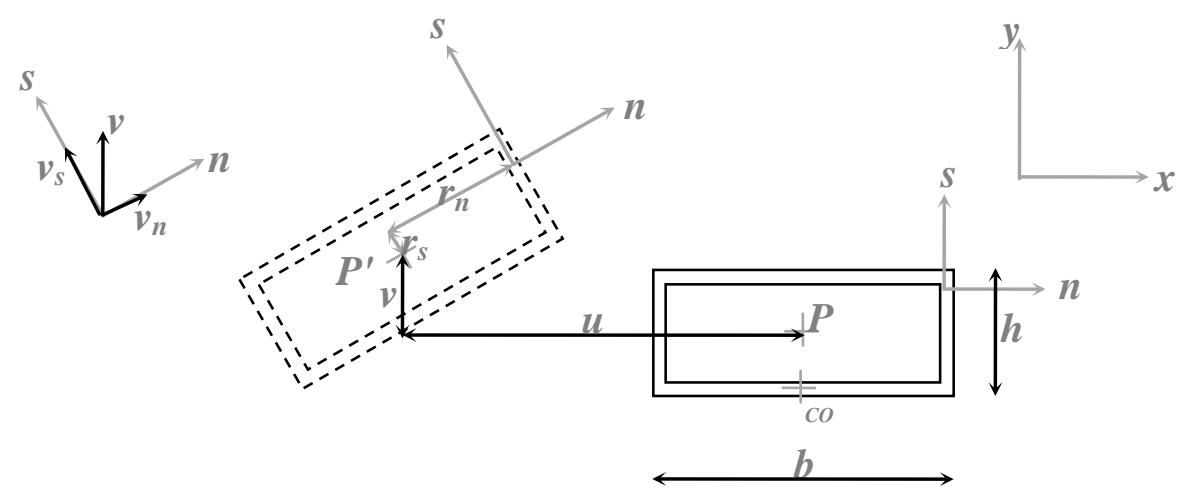

Figure 4.2 Kinematics of a beam cross-section

An additional degree of freedom $\phi(z)$ is introduced as the measure of warping. As in the Benscoter's thin-walled beam theory [8], it is assumed to be independent of the axial rotation degree of freedom of the beam. In terms of these, the tangential and warping displacements can now be written as follows [18]:

$$
\begin{gathered}
v_{s}(s, n, z)=u(z) \frac{\partial x(s)}{\partial s}+v(z) \frac{\partial y(s)}{\partial s}+r_{n}(s, n) \theta_{z}(z) \\
w(s, n, z)=w_{o}(z)+y(s) \theta_{x}(z)-x(s) \theta_{y}(z)-\psi(s, n) \phi(z)
\end{gathered}
$$


where $u(z)$ and $v(z)$ are the displacements of the pole in $\mathrm{x}$ and y directions, respectively; $w(z)$ is the warping displacement of the contour origin; $\theta_{x}(z)$ and $\theta_{y}(z)$ are the rotations of pole with respect to the $x$ and $y$ axis, respectively; $\theta_{z}(z)$ is the angle of twist; $\psi(s, n)$ is the warping function; $\phi(z)$ is the measure of warping that in special cases can be approximated to the rate of twist, $\theta_{z}^{\prime}$; ()'denotes the derivative with respect to $z ; x(s)$ and $y(s)$ are Cartesian coordinate rewritten in term of tangential coordinate. In the linear theory, the rotations of pole are described as follows:

$$
\begin{aligned}
& \theta_{x}(z)=\gamma_{y z}(z)-v^{\prime}(z) \\
& \theta_{y}(z)=\gamma_{x z}(z)-u^{\prime}(z)
\end{aligned}
$$

where $\gamma_{x z}$ and $\gamma_{y z}$ are transverse shear strains. In the shear deformable beam theory, the EulerBernoulli hypothesis is no longer applicable as $\gamma_{x z} \neq 0$ and $\gamma_{y z} \neq 0$ and the transverse strains are included in the derivation. Infinitesimal strain tensor in linear theory is expressed in terms of displacements. Based on the thin-walled theory, the energy equation requires only two main strain components, $\varepsilon_{z z}$ which is the normal strain in $z$ direction and $\gamma_{s z}=2 \varepsilon_{s z}$ which is the shear strain in $s z$ plane. They are defined as follows:

$$
\begin{gathered}
\varepsilon_{z z}=\frac{\partial w}{\partial z} \\
\gamma_{s z}=2 \varepsilon_{s z}=\frac{\partial v_{s}}{\partial z}+\frac{\partial w}{\partial s}
\end{gathered}
$$

Both the open and the two closed cross-sections have the same geometric center but different shear centers, thus, causing the centers of twist to vary along the beam length. Although the beam is subjected only to torsion, it is no longer the case of pure torsion, but the coupled bending and torsion must be considered. The governing equations are derived using the principle of virtual work as follows. The following derivation is based on isotropic materials; however, it could be generalized to use for the composite materials as shown in [22]. In static analysis, the principle of virtual work can be written as follows:

$$
\delta W_{\text {Internal Work }}-\delta W_{\text {External Work }}=0
$$




$$
\iiint_{V} \sigma \delta \varepsilon d V-\left(\iiint_{V} B \delta u d V+\iint_{S} T \delta u d S\right)=0
$$

where $B$ and $T$ stand for body forces and tractions, respectively.

For a thin-walled beam cross-section, the body forces are zero in a static case and the term of work due to strain energy can be simplified. Considering the tractions in the form of distributed loads along the span and concentrated loads at certain locations of the span, the equation of virtual work can be written as follows:

$$
\begin{aligned}
& \int_{a}^{b}\left[\int_{A}\left(E \varepsilon_{z z} \delta \varepsilon_{z z}+G \gamma_{s z} \delta \gamma_{s z}\right) d A\right. \\
& \left.-\left(p_{x} \delta u+p_{y} \delta v+p_{z} \delta w+m_{x} \delta \theta_{y}+m_{y} \delta \theta_{x}+m_{z} \delta \theta_{z}-m_{\omega} \delta \phi\right)\right] d Z \\
& -\left(P_{x} \delta u+P_{y} \delta v+P_{z} \delta w+M_{x} \delta \theta_{x}+M_{y} \delta \theta_{y}+M_{z} \delta \theta_{z}-M_{\omega} \delta \phi\right)=0
\end{aligned}
$$

where $E$ is the Young's modulus and $G$ is the modulus of torsional rigidity; $p_{x}, p_{y}$ and $p_{z}$ are the distributed forces in $x$-, $y$ - and $z$-direction, respectively; $m_{x}, m_{y}$ and $m_{z}$ are the distributed moments with respect to $x, y$ and $z$ axis, respectively; $m_{\omega}$ is the distributed warping torque about $z$ axis; $P_{x}$ and $P_{y}$ are the shear forces in $x$ and $y$ directions, respectively; $P_{z}$ is the axial force; $M_{x}$ and $M_{y}$ are the bending moments with respect to $x$ and $y$ axis, respectively; $M_{z}$ is the torque; and $M_{\omega}$ is the warping moment or so-called "Bimoment".

In the span-wise direction, the beam model consists of three portions. For convenience, the local coordinates are mainly used as the references in the span-wise direction. The first portion has boundaries from $z_{1}=0$ to $z_{1}=l_{1}$, the second portion with the crack spans from $z_{2}=0$ to $z_{2}=l_{2}$ and the last portion spans from $z_{3}=0$ to $z_{3}=l_{3}$. For the plane of cross-section, however, the global coordinates are still used. The contour origin is chosen so that the warping displacement, $w_{o}(z)$, at the contour origin is eliminated. Substituting all stress and strain components and applying the principle of virtual work, the system of equilibrium equations as well as corresponding boundary-continuity conditions are obtained as follows: 


$$
\begin{gathered}
-E A w^{\prime \prime}=p_{z} \\
-G J_{\theta y} \theta_{z}^{\prime \prime}+G Q_{y z} \phi^{\prime}-G Q_{x y}\left(v^{\prime \prime}+\theta_{x}^{\prime}\right)-G Q_{y y}\left(u^{\prime \prime}-\theta_{y}^{\prime}\right)=p_{x} \\
-G J_{\theta x} \theta_{z}^{\prime \prime}+G Q_{x z} \phi^{\prime}-G Q_{x x}\left(v^{\prime \prime}+\theta_{x}^{\prime}\right)-G Q_{x y}\left(u^{\prime \prime}-\theta_{y}^{\prime}\right)=p_{y} \\
E \Gamma_{z x} \phi^{\prime \prime}-E I_{x x} \theta_{x}^{\prime \prime}+E I_{x y} \theta_{y}^{\prime \prime}+G J_{\theta x} \theta_{z}^{\prime}-G Q_{x z} \phi+G Q_{x x}\left(v^{\prime}+\theta_{x}\right)+G Q_{x y}\left(u^{\prime}-\theta_{y}\right)=m_{x} \\
-E \Gamma_{z y} \phi^{\prime \prime}+E I_{x y} \theta_{x}^{\prime \prime}-E I_{y y} \theta_{y}^{\prime \prime}-G J_{\theta y} \theta_{z}^{\prime}+G Q_{y z} \phi-G Q_{x y}\left(v^{\prime}+\theta_{x}\right)-G Q_{y y}\left(u^{\prime}-\theta_{y}\right)=m_{y} \\
-G J_{\theta \theta} \theta_{z}^{\prime \prime}+G J_{\theta z} \phi^{\prime}-G J_{\theta x}\left(v^{\prime \prime}+\theta_{x}^{\prime}\right)-G J_{\theta y}\left(u^{\prime \prime}-\theta_{y}^{\prime}\right)=m_{z} \\
-E \Gamma_{z z} \phi^{\prime \prime}+E \Gamma_{z x} \theta_{x}^{\prime \prime}-E \Gamma_{z y} \theta_{y}^{\prime \prime}-G J_{\theta z} \theta_{z}^{\prime}+G J_{z z} \phi-G Q_{x z}\left(v^{\prime}+\theta_{x}\right)-G Q_{y z}\left(u^{\prime}-\theta_{y}\right)=-m_{\omega}
\end{gathered}
$$

The geometric problem parameters used in the above equations are defined as follows:

$$
\begin{aligned}
& A=\int_{A} d A, S_{x x}=\int_{A} y d A, S_{y y}=\int_{A} x d A, S_{z z}=\int_{A} \psi d A \\
& \Gamma_{z z}=\int_{A} \psi^{2} d A, \Gamma_{z x}=\int_{A} y \psi d A, \Gamma_{z y}=\int_{A} x \psi d A, \\
& I_{x x}=\int_{A} y^{2} d A, I_{x y}=\int_{A} x y d A, I_{y y}=\int_{A} x^{2} d A \\
& J_{\theta \theta}=\int_{A} R_{n}^{2} d A, J_{\theta z}=\int_{A} R_{n} \frac{\partial \psi}{\partial s} d A, J_{z z}=\int_{A}\left(\frac{\partial \psi}{\partial s}\right)^{2} d A, J_{\theta x}=\int_{A} R_{n} \frac{\partial y}{\partial s} d A, J_{\theta y}=\int_{A} R_{n} \frac{\partial x}{\partial s} d A \\
& Q_{x x}=\int_{A}\left(\frac{\partial y}{\partial s}\right)^{2} d A, Q_{x y}=\int_{A} \frac{\partial x}{\partial s} \frac{\partial y}{\partial s} d A, Q_{y y}=\int_{A}\left(\frac{\partial x}{\partial s}\right)^{2} d A, Q_{x z}=\int_{A} \frac{\partial y}{\partial s} \frac{\partial \psi}{\partial s} d A, Q_{y z}=\int_{A} \frac{\partial x}{\partial s} \frac{\partial \psi}{\partial s} d A
\end{aligned}
$$

The corresponding boundary conditions at the two ends of a section are obtained as follows:

At the fixed end,

$$
\begin{gathered}
u=0, v=0, w=0 \\
\theta_{x}=0, \theta_{y}=0, \theta_{z}=0 \\
\varnothing=0
\end{gathered}
$$

At the free end,

$$
G J_{\theta y} \theta_{z}^{\prime}-G Q_{y z} \phi+G Q_{x y}\left(v^{\prime}+\theta_{x}\right)+G Q_{y y}\left(u^{\prime}-\theta_{y}\right)=P_{x}
$$




$$
\begin{gathered}
G J_{\theta x} \theta_{z}^{\prime}-G Q_{x z} \phi+G Q_{x x}\left(v^{\prime}+\theta_{x}\right)+G Q_{x y}\left(u^{\prime}-\theta_{y}\right)=P_{y} \\
E A w^{\prime}=P_{z} \\
-E \Gamma_{z x} \phi^{\prime}+E I_{x x} \theta_{x}^{\prime}-E I_{x y} \theta_{y}^{\prime}=M_{x} \\
E \Gamma_{z y} \phi^{\prime}+E I_{x y} \theta_{x}^{\prime}-E I_{y y} \theta_{y}^{\prime}=M_{y} \\
G J_{\theta \theta} \theta_{z}^{\prime}-G J_{\theta z} \phi+G J_{\theta x}\left(v^{\prime}+\theta_{x}\right)+G J_{\theta y}\left(u^{\prime}-\theta_{y}\right)=M_{z} \\
E \Gamma_{z z} \phi^{\prime}-E \Gamma_{z x} \theta_{x}^{\prime}-E \Gamma_{z y} \theta_{y}^{\prime}=-M_{\omega}
\end{gathered}
$$

At the interface of the uncracked and cracked portions of the beam the boundary conditions are defined in terms of the continuity conditions as described in the following.

\subsubsection{Continuity Conditions}

Due to the different warping functions between closed and open cross-sections, the discontinuity of warping displacements at the interface of the two sections across the beam portion is inevitable. In order to deal with this issue, the thin-walled beam theory is modified by incorporating the technique proposed by Gunnlaugsson and Pedersen $[18,130]$ to include the compatibility conditions in the boundary conditions at the interface. The objective of their technique is to minimize the difference between the warping displacements of the closed and open sections on the two sides of an interface. To facilitate the minimization of the difference, they proposed to include additional constants in the representation of the warping displacement only on one side of the interface, as explained in the following.

At the interface of the open and closed cross-sections, the warping displacement of the element on the right-hand side is rewritten to include the assumed compatibility constants as follows:

$w^{\text {right }}(s, n, z)=w_{o}(z)+y(s) \theta_{x}(z)-x(s) \theta_{y}(z)-\left[d_{1}+d_{2} y(s)-d_{3} x(s)+d_{4} \psi^{\text {right }}(s, n)\right] \phi(z)$

whereas the warping displacement of the right element remains unchanged as follows: 


$$
w^{l e f t}(s, n, z)=\bar{w}(z)+y(s) \theta_{x}(z)+x(s) \theta_{y}(z)-\psi^{l e f t}(s, n) \phi(z)
$$

To calculate the constants and define the compatibility condition, we minimize the following integral norm of the point-wise difference between the warping displacements:

$$
I=\int_{A}\left(w^{l e f t}-w^{r i g h t}\right)^{2} d A
$$

where left and right superscripts denote the closed and open cross sections, respectively.

The necessary conditions for the minimization of this functional provide the compatibility conditions and the following four equations to calculate the constant $d_{i}$ :

$$
\begin{array}{lcl}
\frac{\partial I}{\partial d_{1}}=0: & \int_{A}\left(w^{\text {left }}-w^{\text {right }}\right) d A=0 \\
\frac{\partial I}{\partial d_{2}}=0: & \int_{A}\left(w^{\text {left }}-w^{\text {right }}\right) y d A=0 \\
\frac{\partial I}{\partial d_{3}}=0: & \int_{A}\left(w^{\text {left }}-w^{\text {right }}\right) x d A=0 \\
\frac{\partial I}{\partial d_{4}}=0: & \int_{A}\left(w^{\text {left }}-w^{\text {right }}\right) \psi^{\text {right }} d A=0
\end{array}
$$

On expansion, the above equations lead to the following four simultaneous equations for calculating the constants $d_{i}$ expressed in terms of the integration constants of the beam cross sections.

$$
\begin{aligned}
& d_{1} A^{r i g h t}+d_{2} S_{x x}^{r i g h t}-d_{3} S_{y y}^{r i g h t}+d_{4} S_{z z}^{r i g h t}=S_{z z}^{\text {left }} \\
& d_{1} S_{x x}^{r i g h t}+d_{2} I_{x x}^{r i g h t}-d_{3} I_{x y}^{r i g h t}+d_{4} \Gamma_{z x}^{r i g h t}=\Gamma_{z x}^{l e f t} \\
& d_{1} S_{y y}^{r i g h t}+d_{2} I_{x y}^{\text {right }}-d_{3} I_{y y}^{r i g h t}+d_{4} \Gamma_{z y}^{r i g h t}=\Gamma_{z y}^{\text {left }} \\
& d_{1} S_{z z}^{\text {right }}+d_{2} \Gamma_{z x}^{\text {right }}-d_{3} \Gamma_{z y}^{\text {right }}+d_{4} \Gamma_{z z}^{r i g h t}=\Gamma_{z z l r}
\end{aligned}
$$

where the integration constants of the beam cross section, $A, S_{x x}, S_{y y}, S_{z z}, I_{x x}, I_{x y}, I_{y y}, \Gamma_{z z}, \Gamma_{z x}$ and $\Gamma_{z y}$ are the same as defined earlier after the equilibrium equations, but they are now defined 
separately for the left and right parts of the beam cross sections. Another constant, undefined earlier, is $\Gamma_{z z l r}=\int_{A} \psi^{\text {left }} \psi^{\text {right }} d A$ which utilizes the warping function on the left and right hand side cross sections.

Here, it is noted that all integration constants are calculated using the respective shear centers as references for both the cross-sections. At this step, the constants obtained using the pole as the references cannot be used because of the flexural-torsional coupling. However, in order to obtain the unique values of all constants no matter which element is assumed to be on the left-hand or the right-hand side, an additional normalization needs to be implemented. It requires that the product of both constants $d_{4}$-- one when the closed cross section is on the left and the other when the closed cross section is on the right -- be normalized, which is basically equivalent to the orthogonality as explained in [130]. The compatibility constants can be implemented into the model directly as follows:

$$
\begin{aligned}
&\left.u\right|_{\text {left }}=\left.u\right|_{\text {right }} \\
&\left.v\right|_{\text {left }}=\left.v\right|_{\text {right }} \\
&\left.w\right|_{\text {left }}+\left.d_{1} \phi\right|_{\text {left }}=\left.w\right|_{\text {right }} \\
&\left.\theta_{x}\right|_{\text {left }}+\left.d_{2} \phi\right|_{\text {left }}=\left.\theta_{x}\right|_{\text {right }} \\
&\left.\theta_{y}\right|_{\text {left }}-\left.d_{3} \phi\right|_{\text {left }}=\left.\theta_{y}\right|_{\text {right }} \\
&\left.\theta_{z}\right|_{\text {left }}=\left.\theta_{z}\right|_{\text {right }} \\
&\left.d_{4} \phi\right|_{\text {left }}=\left.\phi\right|_{\text {right }} \\
&\left.P_{x}\right|_{\text {left }}=\left.P_{x}\right|_{\text {right }} \\
&\left.P_{y}\right|_{\text {left }}=\left.P_{y}\right|_{\text {right }} \\
&\left.P_{z}\right|_{\text {left }}=\left.P_{z}\right|_{\text {right }} \\
&\left.M_{x}\right|_{\text {left }}=\left.M_{x}\right|_{\text {right }} \\
&\left.M_{y}\right|_{\text {left }}=\left.M_{y}\right|_{\text {right }} \\
&\left.M_{z}\right|_{\text {left }}=\left.M_{z}\right|_{\text {right }} \\
&-\left.M_{\omega}\right|_{\text {left }}=\left.d_{1} P_{z}\right|_{\text {right }}+\left.d_{2} M_{x}\right|_{\text {right }}+\left.d_{3} M_{y}\right|_{\text {right }}-\left.d_{4} M_{\omega}\right|_{\text {right }}
\end{aligned}
$$




\subsubsection{Aerodynamic Loadings and Divergence}

Although the load-bearing structure is modeled with a thin-walled beam of rectangular shape, the wing is of airfoil shape. In this paper, the wing is assumed to have a symmetric shape of airfoil wrapped around the beam as shown in Figure 4.3, where $A C$ is the aerodynamic center, $C T$, is the center of twist, $c$, is the length of chord and $e$ is the distance as shown in Figure 4.3 This distance, $e$, is in general independent of the angle of attack and assumed to be $15 \%$ of the chord length, that is, $e=0.15 c$ for this calculation. When an airfoil is exposed to airflow with an angle of attack, $\hat{\theta}_{z}$, it experiences an additional lifting force, $l$, acting at the aerodynamic center and a moment about aerodynamic center, $m_{o}$, as shown in Figure 4.3.
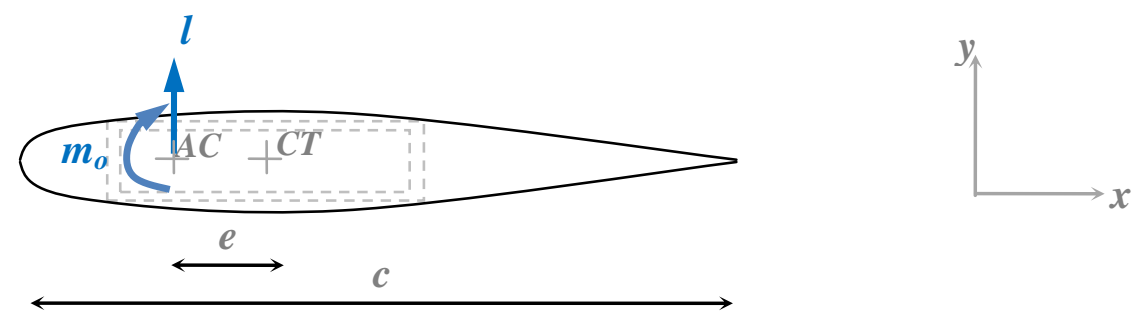

Figure 4.3 Airfoil as a shell around the thin-walled beam wing structure

For the assumed case of the damage symmetrically placed on the top surface of the crosssection, the center of twist is on the same y-axis as the pole for the entire span. In such a damage configuration, the lifting force, $l$, will generate only the twisting moment, $m_{z}$. The lift force and the twisting moments can now be written in terms of the dynamic pressure on the wing and its dimensions as follows:

$$
\begin{gathered}
l(z)=q a c \hat{\theta}_{z}(z) \\
m_{z}(z)=q a c \hat{\theta}_{z}(z) \times e+m_{o}
\end{gathered}
$$

where $q$ is the dynamic pressure and $a$ is the lift-curve slope which is basically the ratio between the lift coefficient and the angle of attack. That is, the product $a \hat{\theta}_{z}(z)$ represents the lift coefficient. 


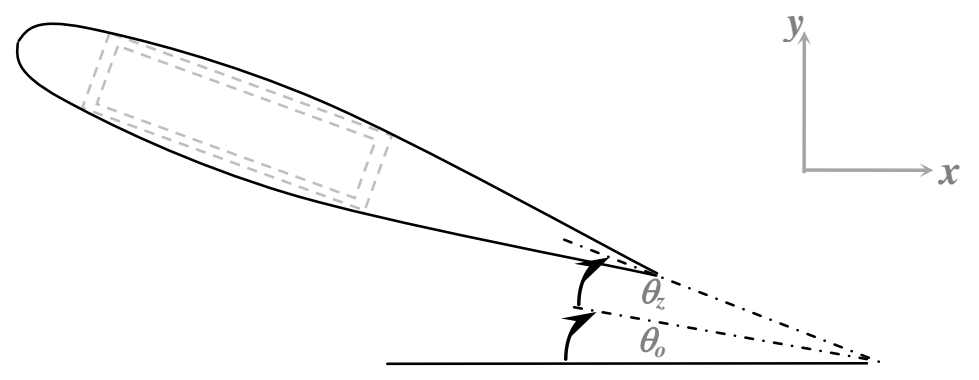

Figure 4.4 Configurations of an airfoil showing the angle of incident and the angle of twist

Figure 4.4 shows that the angle of attack, $\hat{\theta}_{z}$, is sum of the angle of incident, $\theta_{o}$, and the angle of twist, $\theta_{z}$, written as follows:

$$
\hat{\theta}_{z}(z)=\theta_{o}+\theta_{z}(z)
$$

Therefore, assuming the symmetry of airfoil, without any loss of generality, $m_{o}=0$;

$$
m_{z}(z)=\operatorname{qac}\left[\theta_{o}+\theta_{z}(z)\right] \times e
$$

\subsection{Analytical Solutions}

The divergence occurs when the angle of twist starts to grow disproportionately as the twisting moment is increased and the air pressure at which this starts to happen is the divergence dynamic pressure. Since the twisting moment caused by the aerodynamic motion depends not only on the initial incidence angle but also on the wing twist caused by aerodynamic moment acting on the elastic axis, it is necessary to calculate the angle of twist, which in turn depends on the twisting moment acting on the wing. One, thus, needs to use an iterative approach in which one calculates the twisting moment for initial value of the angle of attack followed by the solution of the equilibrium equations to obtain the angle of twist. For small values of the pressure this iterative approach will converge to a final stable solution. However, as the pressure is increased, the final solution for the angle of twist will start to grow disproportionately large thus creating a condition of aeroelastic divergence. This iterative approach can be computationally demanding, but it is necessary for a loosely coupled system, which will be the case for the complicated structural configurations where one must use the finite element approach. Often, an 
eigenvalue analysis, for linear structure and aerodynamics, can be used. In our case, however, we can obtain the divergence dynamic pressure much more efficiently as we can solve the equilibrium equations analytically because of the assumed configuration of the cross section as a thin-walled beam of simple cross section. In the following, we, thus, present a direct one-step approach to calculate the divergence dynamic pressure. This approach also permits us to study the probabilistic characteristics of divergence dynamic pressure for a beam with uncertain damage characteristics. However, before the direct approach is used with the cracked beam in this study, it is first validated for its accuracy by comparing the solution obtained by this approach with that obtained using the iterative approach for a simple beam. In the following section we describe the analytical solution that we have used to calculate the angle of twist needed in the two divergence calculating methods.

To obtain general full-form analytical solution of the seven coupled differential equations, Eq. (4.10) - Eq. (4.16), we rewrite them as a set of coupled first order equations using auxiliary variables and then use Jordan canonical form of the system to decouple the system. The following auxiliary variables: $u_{1}=u^{\prime}, v_{1}=v^{\prime}, \theta_{x 1}=\theta_{x}^{\prime}, \theta_{y 1}=\theta_{y}^{\prime}, \theta_{z 1}=\theta_{z}^{\prime}, \phi_{1}=\phi^{\prime}$ are introduced where ( )'denotes the derivative with respect to $z$. The equation for the displacement, $w$, in $z$-direction is already uncoupled and, thus, it is not included in the analysis. The state vector of variables is defined as follows:

$$
\underline{X}=\left[\begin{array}{llllllllllll}
u & u_{1} & v & v_{1} & \theta_{x} & \theta_{x 1} & \theta_{y} & \theta_{y 1} & \theta_{z} & \theta_{z 1} & \phi & \phi_{1}
\end{array}\right]^{T}
$$

With the assignment of auxiliary variables as indicated above, the coupled equations can be rewritten in the state form as follows:

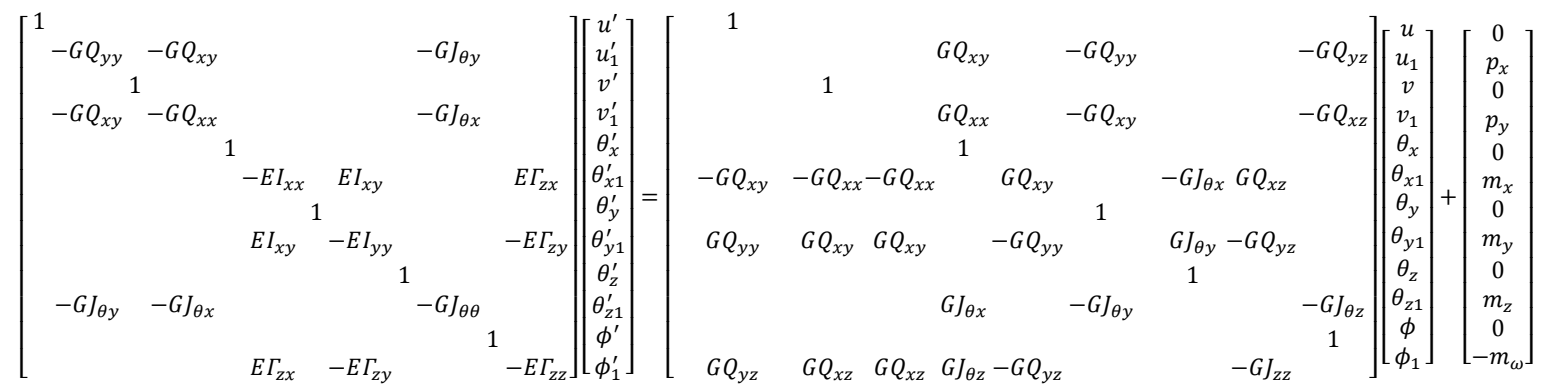


Substituting Eq. (4.55) into Eq. (4.56) to introduce the aero-elastic twisting moment and transferring the force terms dependent on the twisting angle to the left hand side we obtain:

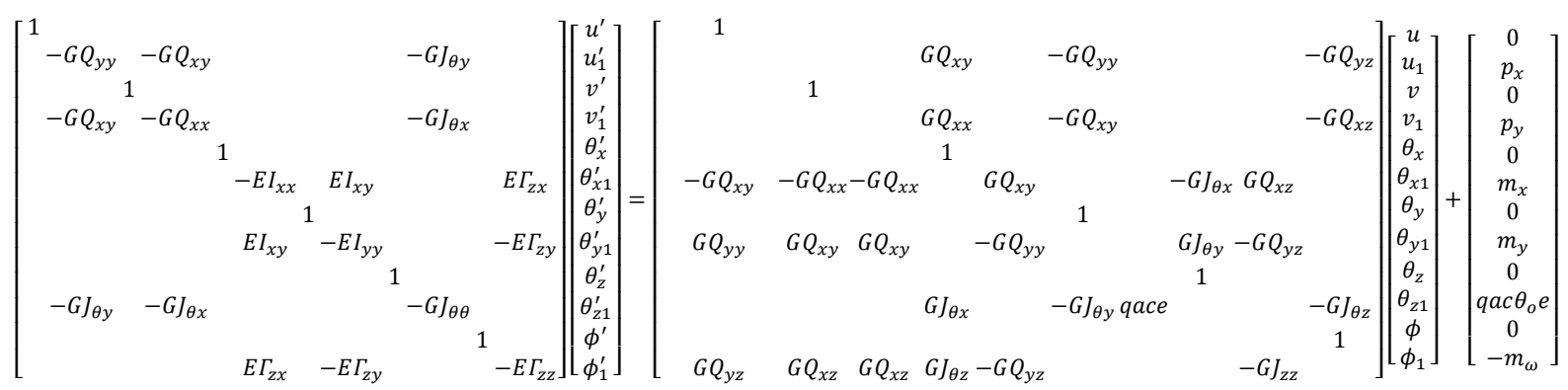

Equation (4.57) yields the $1^{\text {st }}$ order ODE equation in the following form

$$
\begin{gathered}
\underline{A} \underline{X^{\prime}}=\underline{B} \underline{X}+\underline{F} \\
\underline{X^{\prime}}=\underline{A}^{-1} \underline{B} \underline{X}+\underline{A}^{-1} \underline{F}
\end{gathered}
$$

To obtain the solution, the technique of Jordan canonical form [132] is applied. Jordan matrix and the corresponding matrix of transformation are represented by $J$ and $\Phi$, respectively. Let's define

$$
\underline{X}=\underline{\Phi} \underline{Q}
$$

Substitute Eq. (4.60) into Eq. (4.59), we obtain:

$$
\underline{\Phi} \underline{Q}^{\prime}=\underline{A}^{-1} \underline{B} \underline{\Phi} \underline{Q}+\underline{A}^{-1} \underline{F}
$$

Pre-multiply Eq. (4.61) by $\underline{\Phi}^{-1}$, we obtain:

$$
\underline{\Phi}^{-1} \underline{\Phi} \underline{Q}^{\prime}=\underline{\Phi}^{-1} \underline{A}^{-1} \underline{B} \underline{\Phi} \underline{Q}+\underline{\Phi}^{-1} \underline{A}^{-1} \underline{F}
$$

which is re-written in standard form as follows: 


$$
\underline{Q}^{\prime}=\underline{J} \underline{Q}+\underline{E}
$$

where $\underline{\Phi}^{-1} \underline{\Phi}=\underline{I}, \underline{\Phi}^{-1} \underline{A}^{-1} \underline{B} \underline{\Phi}=\underline{J}$ and $\underline{\Phi}^{-1} \underline{A}^{-1} \underline{F}=\underline{E}$.

The final solution of ODE in Eq. (4.63) is typically written as the combination of the homogeneous and particular solutions as follows:

$$
\underline{X}=\underline{X}_{P}+\underline{X}_{H}
$$

The particular and homogeneous solution in this equation can be expressed as follows:

$$
\begin{gathered}
\underline{X}_{P}=\underline{\Phi} \underline{M(z)} \int_{0} \frac{M(z)^{-1}}{E} d z \\
\underline{X}_{H}=\underline{\Phi} \underline{M(z)} \underline{c}
\end{gathered}
$$

where $M(z)$ and $\underline{c}$ are the matrix of functions and the vector of the constants of integration, respectively. The details of various matrices and vectors are provided in the Appendix A. Substituting these forms of particular and homogeneous solutions in Eq. (4.64), we obtain the following for the complete solution:

$$
\underline{X}=\underline{\Phi}[\underline{I(z)}+\underline{d(z)}]
$$

where $\underline{I(z)}=\underline{M(z)} \int_{0} \underline{M(z)^{-1}} \underline{E} d z$ and $\underline{d(z)}=\underline{M(z)} \underline{c}$.

Using this solution all response quantities related to the state vector can thus be written in analytical form. For example, if we are interested in the angle of twist and deflection, say in the $i^{\text {th }}$ beam section we can obtain them from the following equations:

$$
\begin{aligned}
& \theta_{z}^{(i)}=\sum_{k=1}^{n} \Phi_{7 k}^{(i)}\left[I_{k}^{(i)}(z)+d_{k}^{(i)}(z)\right] \\
& u^{(i)}=\sum_{k=1}^{n} \Phi_{1 k}^{(i)}\left[I_{k}^{(i)}(z)+d_{k}^{(i)}(z)\right]
\end{aligned}
$$


where $n$ is the dimension of the state equation which in this case are $n=12$.

To obtain the constants of integration in vector $\underline{c}$, we need to apply the boundary and continuity conditions in each portion of the beam. For each section, the vector $\underline{c}$ consists of 12 of the constant of integration. Thus, for a three section beam there are 36 such constants of integration. In addition there are 4 constants, $d_{i}$, that were introduced in enforcing the warping displacement compatibilities at each section interface. With 2 section interfaces, this introduced 8 more constant to be calculated. Thus a three section beam will require the calculation of a total of 44 constants. The 6 boundary equations, Eq. (4.17) to Eq. (4.19) at the fixed end, 6 boundary equations, Eq. (4.20) to Eq. (4.26) excluding Eq. (4.22) at the free end, 4 minimization conditions, Eq. (4.34) to Eq. (4.37) at each interface and 12 additional conditions in equations, Eq. (4.38) to Eq. (4.51) excluding Eq. (4.40) and Eq. (4.47) at each interface provide just the right number of equations to provide the all constants of integration in vector $\underline{c}$. Application of these boundary conditions leads to the following simultaneous equations to solve for the constants.

$$
\underline{G} \underline{c}=\underline{F_{G}}
$$

The details of the elements of the matrix $\underline{G}$ and vectors $\underline{c}$ and $\underline{F}_{G}$ in Eq. (4.70) are provided in the Appendix B. Knowing these constants, one can obtain any quantity of interest that can be defined in terms of the state vectors.

Once the angle of twist is calculated either by numerical approach or analytically as explained above, one can calculate the divergence dynamic pressure by an iterative approach or by a onestep direct approach as explained in the following paragraphs.

\subsubsection{Pressure at Divergence: Iterative Approach}

As mentioned earlier, in the iterative approach one first calculate the twisting moment based on the initial angle of incidence and applies it as the input to the system to compute the resulting angle of twist. This calculated angle of twist is then used to augment the angle of incidence for which a new twisting moment is calculated and applied to the system as the input in the second 
step of the iteration. In such successive steps one then examines the trend in the change of the angle of twist. If the change in the angle of twist in successive steps gets smaller, then the applied pressure is below the divergence dynamic pressure and the successive steps will converge to the final angle of twist. However, if the change in the angle of twist in successive steps tends to increase then a divergence condition has occurred and in this situation the total angle of twist will tend to become large without any convergence. Thus the critical pressure can be defined as the condition at which the change in the angle of twist is two successive steps are equal to each other. With the solution approach described above, we thus use the following steps to calculate the critical pressure at which this occurs.

Cycle no.1: Define the applied twisting moments assuming the angle of attack equal to the initial angle of incidence as $m_{z}(z)=q a c \theta_{o} \times e$. Solve equilibrium equations for the angle of twist, $\theta_{z 1}(z)$, at a convenient location on the beam in terms of unknown pressure, $q$.

Cycle no.2: For the next step, express the new twisting moment in terms of the augmented angle of attack using the angle of twist calculated in the previous step as $m_{z}(z)=q a c\left[\theta_{o}+\right.$ $\left.\theta_{z 1}(z)\right] \times e$. Solve the equilibrium equations for the new angle of twist, $\theta_{z 2}(z)$, for the second iterative step in terms of the unknown pressure, $q$.

Cycle no.3: For the third step, express the new twisting moment in terms of the new augmented angle of attack using the angle of twist calculated in the previous step as $m_{z}(z)=q a c\left[\theta_{o}+\right.$ $\left.\theta_{z 2}(z)\right] \times e$. Solve the equilibrium equations for the new angle of twist, $\theta_{z 3}(z)$, for the third iterative step in terms of the unknown pressure, $q$.

The critical pressure at which the divergence will occur is then defined by the condition that the increase in the angle of twist in the two successive steps are at least equal. That is, the critical dynamic pressure, $q$, at divergence is the first root of the following equation.

$$
\theta_{z 2}(q)-\theta_{z 1}(q)=\theta_{z 3}(q)-\theta_{z 2}(q)
$$

which can also be written as follows: 


$$
2 \theta_{z 2}(q)=\theta_{z 3}(q)+\theta_{z 1}(q)
$$

\subsubsection{Pressure at Divergence: Direct Approach}

The more direct one-step approach utilizes the condition that at divergence the angle of twist would keep increasing till it becomes unbounded. That is, the divergence begins as soon as the angle of attack mathematically approaches the value of infinity. This condition can be stated in the following form.

$$
\frac{1}{\theta_{z}(z)}=0
$$

The first root of this equation provides the dynamic pressure at divergence.

\subsection{Numerical Results}

To obtain the numerical results, we consider a beam with a longitudinal crack which is symmetrically located on one of its faces. With such symmetry, several terms in the equation of equilibrium become zero. For the system with span-wise $y-y$ symmetry, the geometric parameters of the cross section, $Q_{x y}, I_{x y}, J_{\theta x}, J_{\theta y}$ and $Q_{x z}$, are all identically zero. For convenience, we choose the contour origin to be on the axis of symmetry so the warping displacement of the contour origin can be also dropped. This means that the points on the contour origins along the span do not warp due to the fact that every point along the fold of symmetry is fixed. This simplifies the equation considerably to the following set.

$$
\begin{gathered}
-G Q_{y y}\left(u^{\prime \prime}-\theta_{y}^{\prime}\right)=p_{x} \\
-E \Gamma_{z y} \phi^{\prime \prime}-E I_{y y} \theta_{y}^{\prime \prime}-G Q_{y y}\left(u^{\prime}-\theta_{y}\right)=m_{y} \\
-G J_{\theta \theta} \theta_{z}^{\prime \prime}+G J_{\theta z} \phi^{\prime}=m_{z} \\
-E \Gamma_{z z} \phi^{\prime \prime}-E \Gamma_{z y} \theta_{y}^{\prime \prime}-G J_{\theta z} \theta_{z}^{\prime}+G J_{z z} \phi=-m_{\omega}
\end{gathered}
$$

From this set the equations of the beam displacement along the $z-z$ and $y-y$ axis are omitted, as they are uncoupled from this set and can be integrated separately. This reduced set of equations can be readily decoupled as explained earlier, to obtain the analytical solution for each degree of freedom, as long as all distributed loadings are described as integrable functions. 
To obtain the numerical results, the length of thin-walled beam, $L$ is assumed to be $6 \mathrm{~m}$, with cross sectional dimensions of width $=0.4 \mathrm{~m}$, depth $=0.2 \mathrm{~m}$ and thickness $=0.01 \mathrm{~m}$. Let the modulus of elasticity $E=71.7 \mathrm{GPa}$ and $v=0.33$. The numerical expressions of warping functions used herein are as follows:

$$
\begin{gathered}
\psi_{c}(s)= \begin{cases}-0.0067+0.033(-0.4-s), & -0.6 \leq s<-0.4 \\
0.0067-0.067(-0.2-s), & -0.4 \leq s<-0.2 \\
-0.033 s \quad,-0.2 \leq s<0.2 \\
-0.0067+0.067(-0.2+s), & 0.2 \leq s<0.4 \\
0.0067-0.033(-0.4+s), & 0.4 \leq s<0.6\end{cases} \\
\psi_{o}(s, n)= \begin{cases}-0.012-0.34(-s-0.4)-n(s+0.6),-0.6 \leq s<-0.4 \\
0.028-0.2(-s-0.2)-n(s+0.06),-0.4 \leq s<-0.2 \\
-0.14 s-n s \quad, \quad-0.2 \leq s<0.2 \\
-0.028+0.2(s-0.2)-n(s-0.06), \quad 0.2 \leq s<0.4 \\
0.012+0.34(s-0.4)-n(s-0.6), \quad 0.4 \leq s<0.6\end{cases}
\end{gathered}
$$

The plots of these warping functions are shown in, meters, in Figure 4.5.

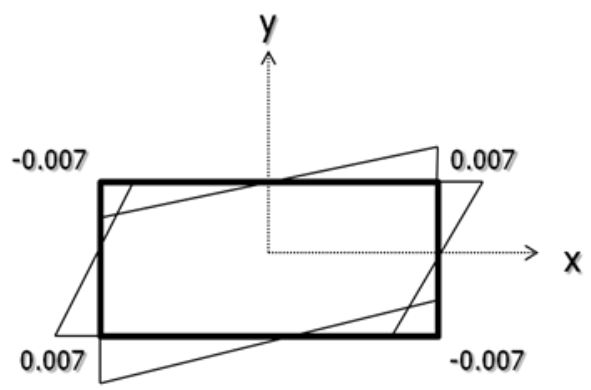

a) Closed cross-section

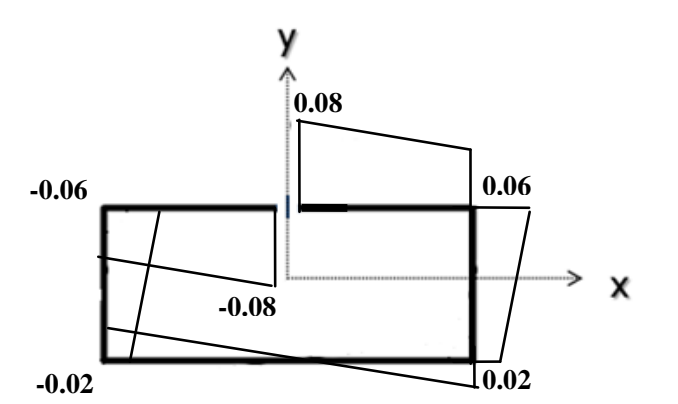

b) Open cross-section

Figure 4.5 Numerical plots of warping functions used in the study. 
For this particular section, it is found that the compatibility constants have the following values, $d_{1}=d_{2}=0, d_{3}=-0.0294$, and $d_{4}=0.122$, the open cross-section being on the right-hand side while $d_{3}=0.240$ and $d_{4}=8.16$ when the open cross-section is on the left-hand side.

For aerodynamic configurations, the length of chord, $c$, is assumed equal to twice the beam width that is $c=2 b$ and thus $e=0.3 b$. The lift-curve slope, $a$, is assumed to be 0.1 per degree. The choice of the angle of incidence, $\theta_{o}$, is arbitrary.

\subsubsection{Validation}

To validate the proposed methodologies for predicting the aeroelastic divergence pressure, a pristine structure without a single crack is first investigated. In this particular case, there exists an empirical approach. Although its derivation does not contain the component of warping, it is expected to yield a relatively good result since the effect of warping restraint at the fixed end is minimized for the thin-walled beam with a closed-section. The dynamic pressure at the divergence or so-called divergence dynamic pressure from this empirical approach is given as follows $[2,140]$ :

$$
q_{\text {div }}=\frac{\pi^{2} G J}{4 e c l^{2} a}
$$

where $J$ is the polar moment of inertia of the cross-section.

Table 4.1 provides results from three different methods of obtaining the divergence dynamic pressure. Comparisons of the results indicate consistency.

Table 4.1 Divergence dynamic pressures $\left(q_{\text {div }}\right)$ obtained by different approaches

\begin{tabular}{cl}
\hline \hline Method & $q_{\text {div }}$ (Pascal) \\
\hline Empirical formula & $7.1662 \times 10^{5}$ \\
Iterative approach & $7.1795 \times 10^{5}$ \\
Direct approach & $7.2040 \times 10^{5}$ \\
\hline \hline
\end{tabular}




\subsubsection{Parametric Investigation}

In the following, we present some results showing the impact of the crack parameters on the calculated divergence dynamic pressure. The crack parameters of interest are the crack location and crack dimensions. The crack location is defined by the parameter, $L_{c}$ which is the location of the center of the crack along the beam length and the crack size, $L_{d}$.

Figures 4.6, 4.7 and 4.8 show the effect of the crack size and crack location on the divergence dynamic pressure, $q_{d i v}$, in different forms. In Figure 4.6, we show the divergence dynamic pressure for cracks located at three distinct positions of $L / 4, L / 2$ and $3 L / 4$ along the span with crack size varying within the range of 0 to $20 \%$. The same results are presented in normalized form in Figure 4.7 where the divergence dynamic pressure for the cracked beam is normalized by the pressure for the same beam without any crack. In Figure 4.8, we plot that as a function of the crack location along the span for three distinct crack sizes. All these figures clearly indicate that the presence of a crack lowers the divergence dynamic pressure compared to the pressure for an uncracked beam. Secondly, we observe that a larger crack lowers the pressure more; that is, a beam with a larger crack will reach the divergence at lower dynamic pressures than a beam with a smaller crack. Furthermore, a crack near the fixed end lowers the divergence dynamic pressure more than a crack farther away from the fixed end; that is, a crack near the root makes the beam more susceptible to the divergence than the crack farther away from the root. These observations are quite understandable as the larger cracks and the cracks closer to the root reduce the stiffness of the beam more, thus making it more susceptible to divergence. 


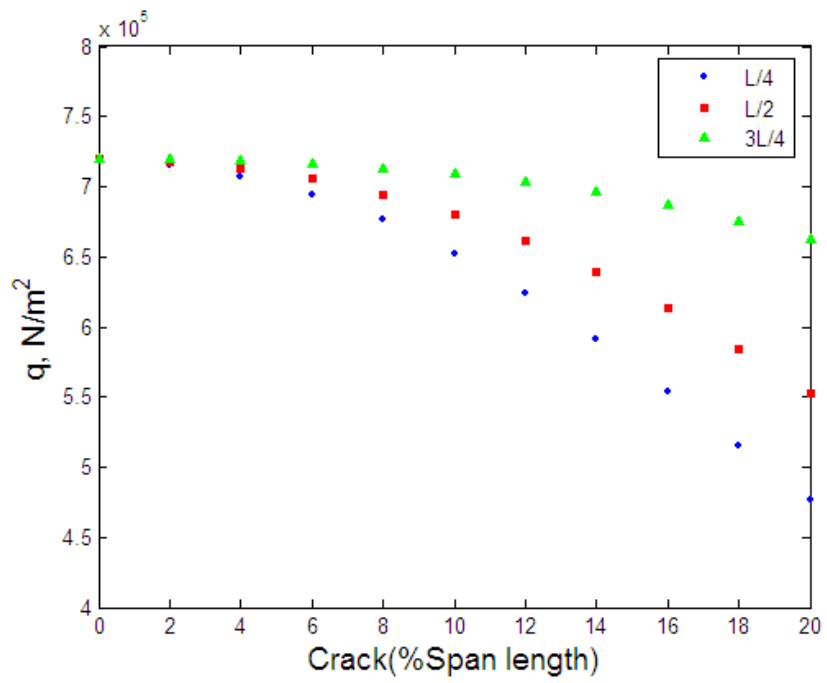

Figure 4.6 Divergence dynamic pressures for different crack sizes located at three different positions along the beam

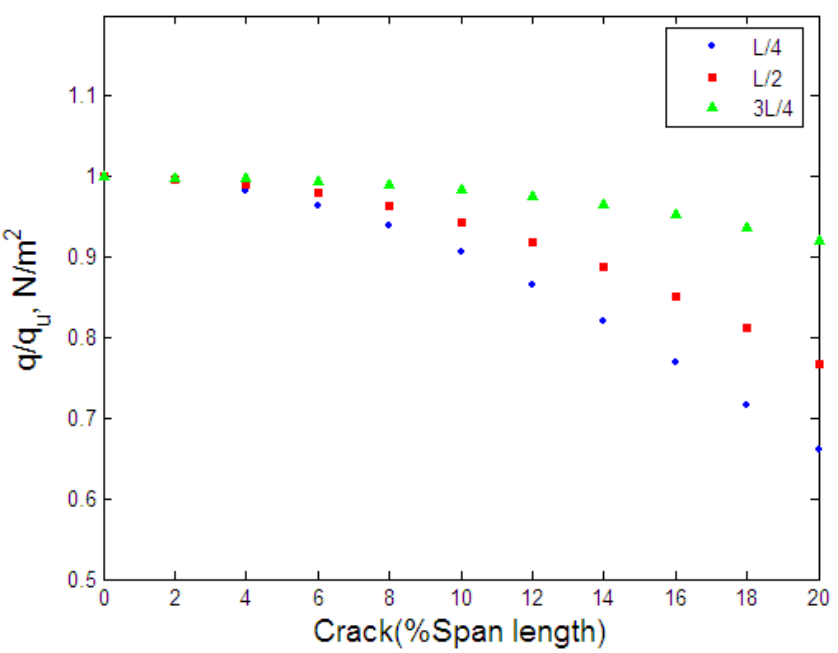

Figure 4.7 Normalized divergence dynamic pressures for different crack sizes located at three different positions along the beam 


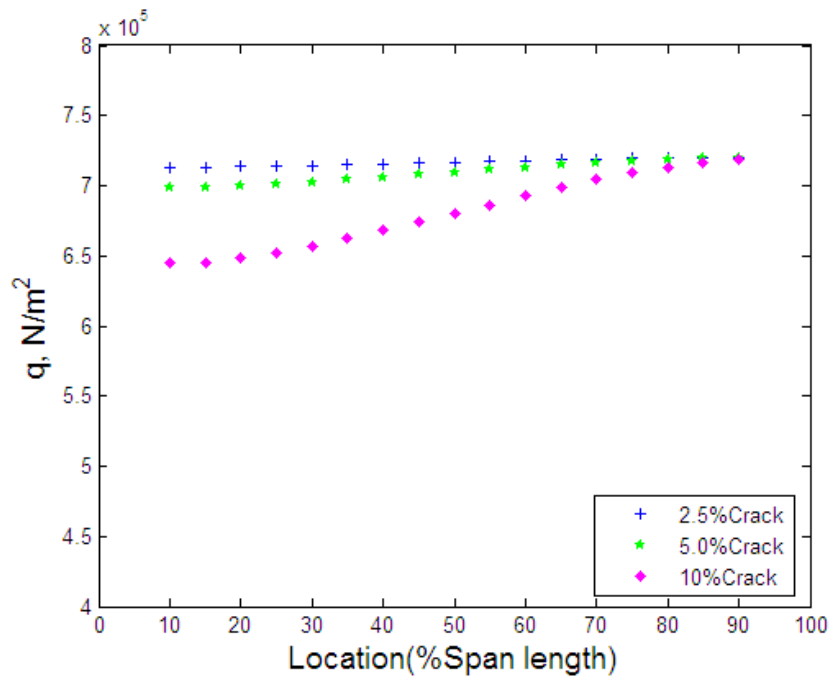

Figure 4.8 Divergence dynamic pressures for three different size cracks located at different locations along the beam.

\subsubsection{Probabilistic Characteristics of the Divergence Dynamic Pressure}

In the following, we present some Monte Carlo simulation results obtained for probabilistically defined crack parameters. Given that the location and size of the crack are Gaussian variables, Figures 4.9, 4.10 and 4.11 shows the statistical distribution of the divergence dynamic pressure in form of a histogram of 500 samples for various sets of distribution parameters. 


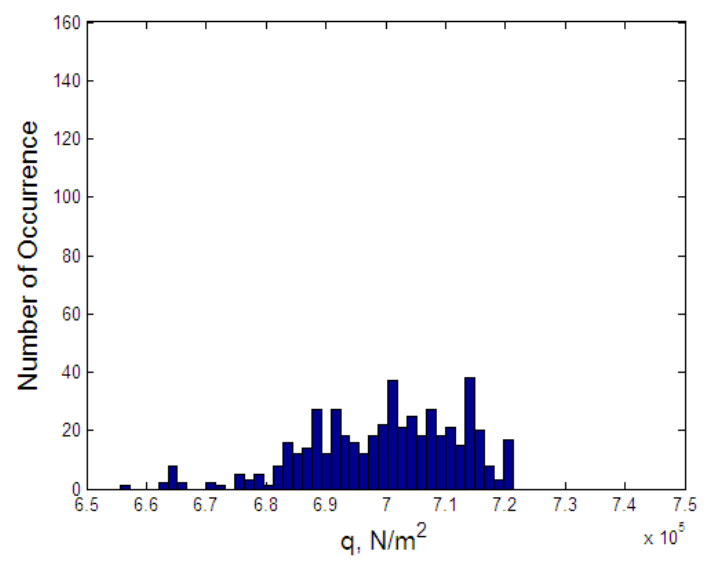

a) $L_{d}: N(0.3,0.15) m$

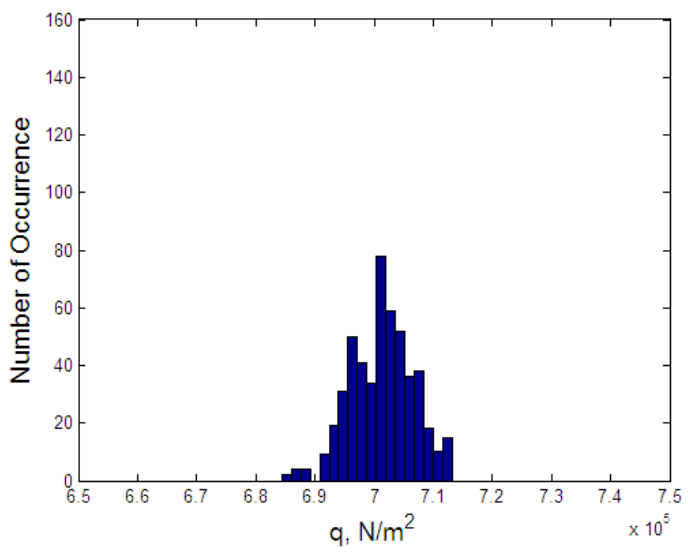

b) $L_{d}: N(0.3,0.06) m$

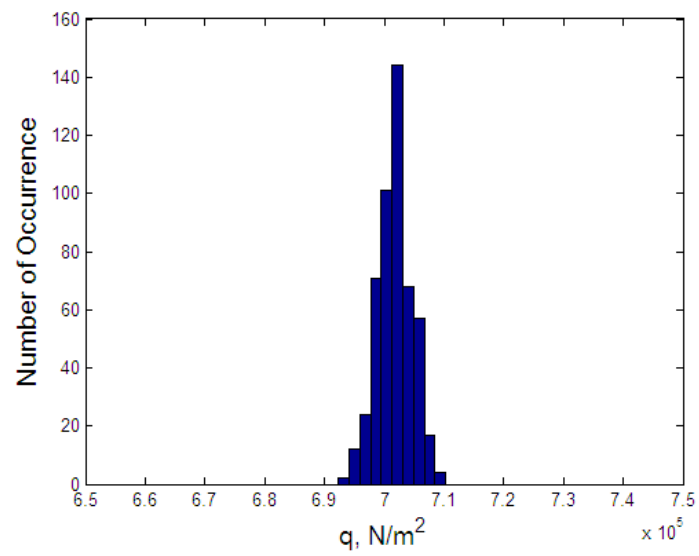

c) $L_{d}: N(0.3,0.03) m$

Figure 4.9 Distributions of divergence dynamic pressures for the crack location, $L_{c}: N(1.5,0.375)$ and three different distributions of crack sizes 


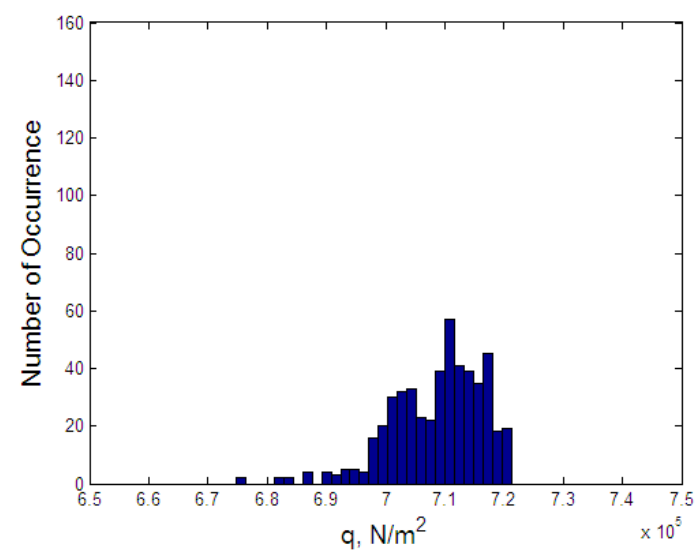

a) $L_{d}: N(0.3,0.15) m$

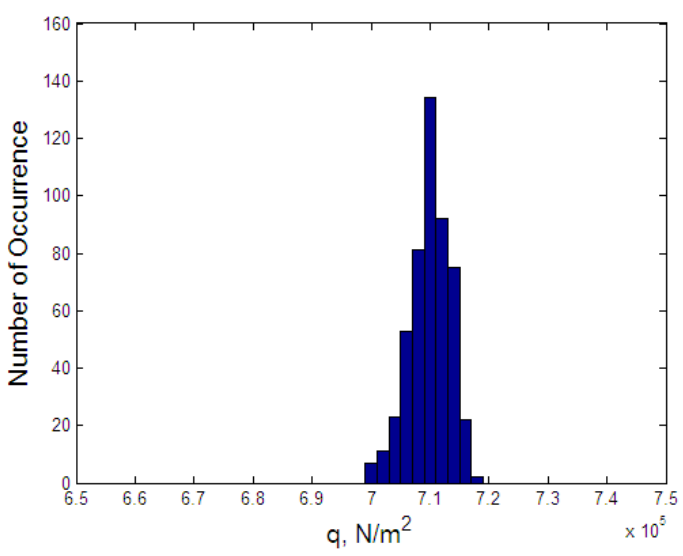

b) $L_{d}: N(0.3,0.06) m$

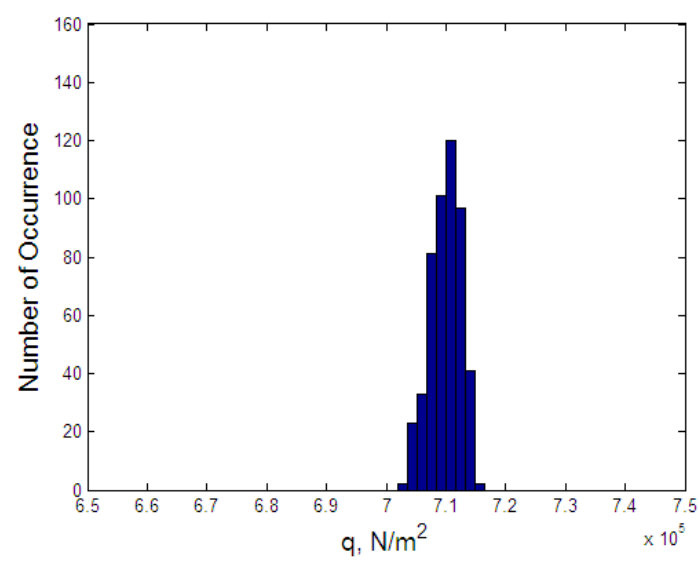

c) $L_{d}: N(0.3,0.03) m$

Figure 4.10 Distributions of divergence dynamic pressures for the crack location, $L c: N(3.0,0.375)$ and three different distributions of crack sizes 


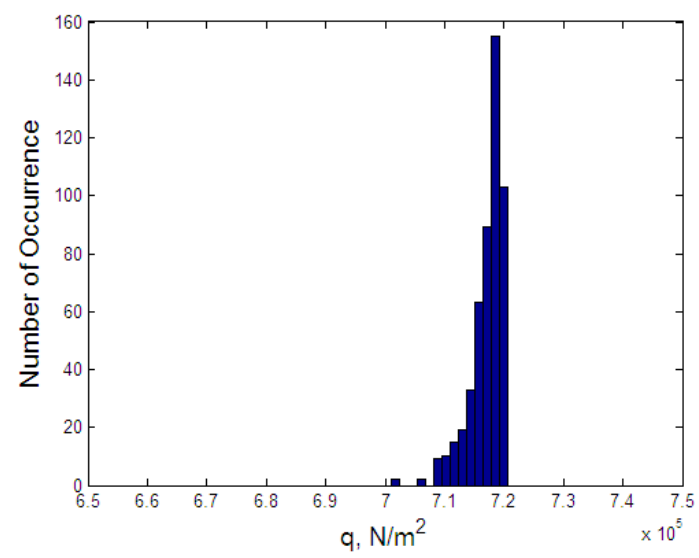

a) $L_{d}: N(0.3,0.15) m$

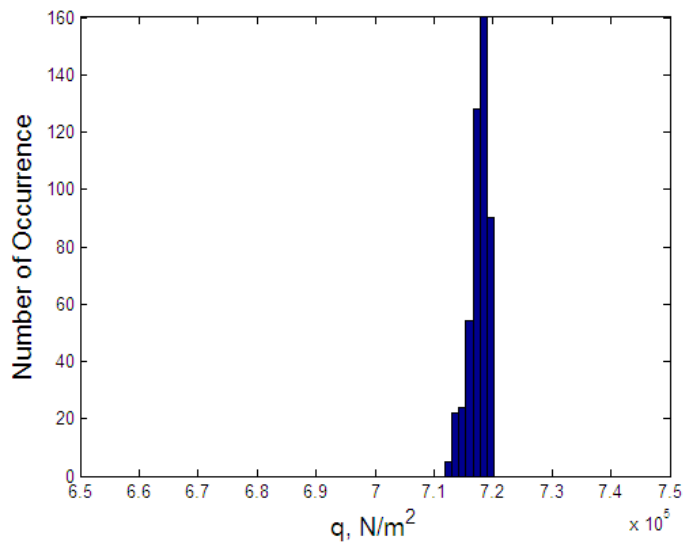

b) $L_{d}: N(0.3,0.06) m$

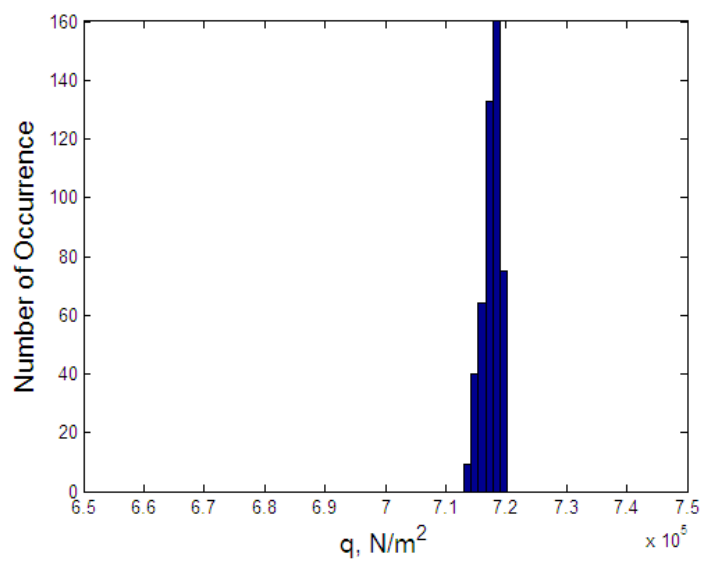

c) $L_{d}: N(0.3,0.03) m$

Figure 4.11 Distributions of divergence dynamic pressures for the crack location, $L c: N(4.5,0.375)$ and three different distributions of crack sizes 
For the histograms shown in Figures 4.9 to 4.11, the statistics of the divergence dynamic pressure were calculated and are given in Table 4.2. Figures 4.9 - 4.11 show an interesting trend in the skewness of the divergence dynamic pressure distribution. We observe that the distributions are more skewed when we have a large coefficient of variation and also when the crack location is farther away from the fixed end. It is primarily because for very small crack sizes (which occur in the crack size samples more frequently for distributions with large variability) or for cracks near away from the fixed end, the effect of the crack on the divergence dynamic pressure value is rather small and then the divergence dynamic pressure is essentially the same as the divergence dynamic pressure for an uncracked beam. This value of the divergence dynamic pressure for the chosen cross section is equal $7.2040 \times 10^{5} \mathrm{~Pa}$. The calculated skewness coefficient values are shown in Table 4.2 which clearly indicates the above observed trend. This trend is also apparent from the figures of the histogram. This skewness of the distribution affects the calculated sample mean and coefficient of variation values. The mean values of the divergence dynamic pressures for cracks near the free end are closer to the crackfree divergence value. The distributions for this case are more skewed towards this crack free divergence value with large skewness coefficient and smaller coefficient of variation. As we approach the fixed end the mean and skewness coefficient values go down and the coefficient of variation does not have a clear trend. 
Table 4.2 Statistics measures of divergence dynamic pressures due to various combinations of crack parameters

\begin{tabular}{ccccc}
\hline \multicolumn{2}{c}{ Crack parameters } & Mean & $\begin{array}{c}\text { Coefficient } \\
\text { of variation }\end{array}$ & Skewness \\
\cline { 1 - 4 } Location, $L_{c}(m)$ & Size, $L_{d}(m)$ & & 0.0180 & -0.6130 \\
$\mathrm{~N}(1.5,0.375)$ & $\mathrm{N}(0.3,0.15)$ & 6.9978 & 0.0074 & -0.1637 \\
$\mathrm{~N}(1.5,0.375)$ & $\mathrm{N}(0.3,0.06)$ & 7.0137 & 0.001 & -0.0229 \\
$\mathrm{~N}(1.5,0.375)$ & $\mathrm{N}(0.3,0.03)$ & 7.0178 & 0.0041 & -0.9180 \\
$\mathrm{~N}(3.0,0.375)$ & $\mathrm{N}(0.3,0.15)$ & 7.0859 & 0.0110 & -0.4553 \\
$\mathrm{~N}(3.0,0.375)$ & $\mathrm{N}(0.3,0.06)$ & 7.0993 & 0.0049 & -0.2834 \\
$\mathrm{~N}(3.0,0.375)$ & $\mathrm{N}(0.3,0.03)$ & 7.0992 & 0.0035 & -1.7485 \\
$\mathrm{~N}(4.5,0.375)$ & $\mathrm{N}(0.3,0.15)$ & 7.1716 & 0.0040 & -0.9611 \\
$\mathrm{~N}(4.5,0.375)$ & $\mathrm{N}(0.3,0.06)$ & 7.1761 & 0.0022 & -0.6706 \\
$\mathrm{~N}(4.5,0.375)$ & $\mathrm{N}(0.3,0.03)$ & 7.1758 & 0.0020 & \\
\hline
\end{tabular}

Using the simulated divergence dynamic pressure values, we also calculate the probability of the divergence dynamic pressure, $q$, falling below a certain value. We call this probability as the probability of failure. This limiting value of the divergence dynamic pressure was chosen to be a factor (less than 1) times the divergence dynamic pressure, $q_{\text {div }}$ of undamaged beam. In Figures 4.12a, $4.12 \mathrm{~b}$ and $4.12 \mathrm{c}$ are shown the plots of these probabilities of failure for three different locations of damage at $\mathrm{L} / 4, \mathrm{~L} / 2$ and $3 \mathrm{~L} / 4$, respectively. The two curves are very close to each other because their coefficients of variation are rather close to each other. The probability curve is flatter for the case with larger coefficient of variation as in this case there are several samples with large crack size values that reduce the divergence dynamic pressure. This is indicated by the left hand portions in Figures 4.12. At the same time for large coefficient of variation case, there are several crack size samples that have very small values for which the divergence dynamic pressure approaches the value $q_{d i v}$ for the uncracked beam. It is for this reason, the probability values for this case are lower than for the other two cases, as show in the right hand portion of Figures $4.12 \mathrm{a}$ and $4.12 \mathrm{~b}$. The curves for all three crack size distributions approach each other for the cracks located near the free end, as observed in Figure 4.12c and to some extent in Figure $4.12 \mathrm{~b}$ as well. 


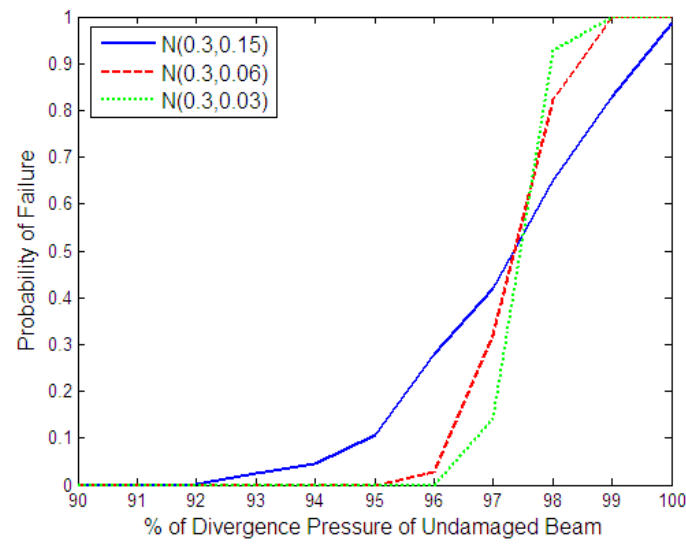

a) $L / 4$

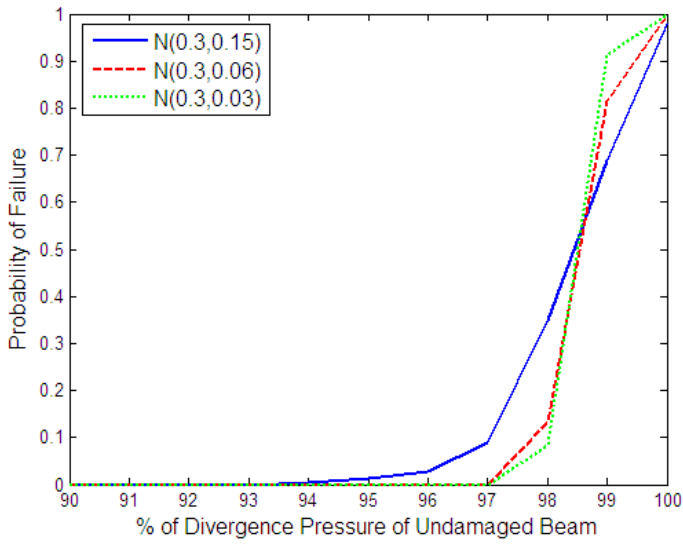

b) $L / 2$

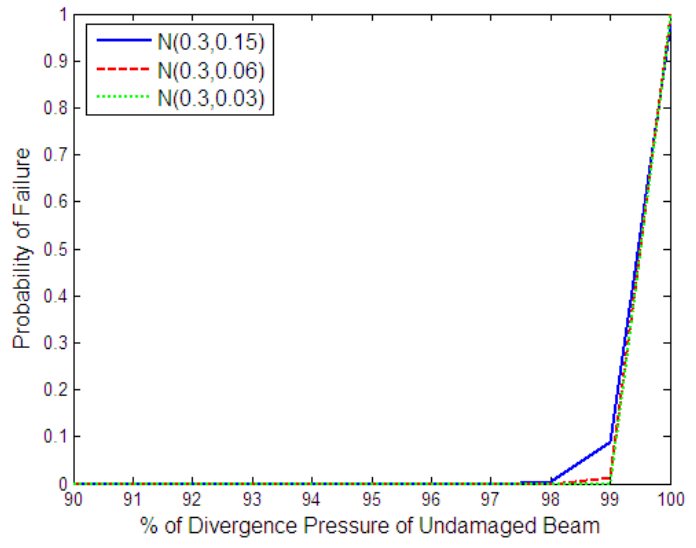

c) $3 \mathrm{~L} / 4$

Figure 4.12 Probability of divergence dynamic pressure for cracked beam being less than a factor time the divergence dynamic pressure of uncracked beam for three different coefficients of variation of crack size 


\subsection{Summary and Concluding Remarks}

The paper presents an analytical approach to determine the divergence of a thin walled beam with a crack oriented along the beam length. The governing equations and associated boundary conditions of the structural model are derived using the principle of virtual work. To obtain the analytical solution, the coupled system equations are first expressed in the state-space form and then are decoupled using the Jordan canonical form. The uncoupled equations are then solved using standard approach. The formulations for calculating the divergence dynamic pressure by the iterative and direct approaches that utilize the analytical solutions are presented. These formulations are used to conduct a parametric study showing the effect of the crack parameters on the divergence dynamic pressure as well as to investigate the effect of uncertainty in the crack parameters in a probabilistic sense. 
Chapter 5: Summary and Concluding Remarks 


\section{Conclusions}

\subsection{General Summary}

In this chapter we summarize the study presented in the previous four chapters. The primary motivation of this study was to evaluate the effect of discrete source damage that an aircraft might experience on its wing structure during its operation. Discrete source damage could occur due to different reasons and could also take widely different forms and configurations. To limit the scope of the work and to conduct the study without detailed finite element modeling of the wing structure, here a simple thin-walled beam with a simple longitudinal crack on one of its surfaces is examined with the hope that this study will establish a simple computational frame work to study this problem in possibly more details for more realistic structural configurations of the wings with damage. As the size and location of the damage are likely to be uncertain, the study develops a framework to incorporate these uncertainties in the analysis to study their impact on static and dynamic responses, time growth of the crack and aero-elastic divergence instabilities.

In Chapter 2, first the analytical modeling of the thin-walled beam with a crack is described. After a careful study of the available literature on the modeling of thin-walled beam, a model based on Benscoter [8] thin-walled beam theory is adopted to account for warping deformations properly. To handle the issue of discontinuities between different cross-sections due to the existence of a crack, some adjustments to the classical theory of thin-walled beam are required. Unlike modified theories such as hybrid elements or special warping functions, the concept of least-square compatibility conditions proposed by Gunnlaugsson and Pedersen [18, 20, 130] is used directly into the boundary value problem obtained from a classical thin-walled theory, Benscoter's beam. The equilibrium equations are developed using the principle of virtual work. Without exception, the general form of the exact solution can be obtained for these equilibrium equations using a combination of the state variables representation and the Jordan canonical form. The validity of the proposed analytical approach is established by comparing the numerical response results obtained by the approach with those obtained by detailed shell finite element analysis using ABAQUS ${ }^{\circledR}$. Characteristics of the damage are uncertainty quantities. The analytical solution is used in the probabilistic analysis where for a crack with random location 
and size the probability of the cracked beam response exceeding a limit state are calculated using the first order safety index analysis. Such analyses can be beneficial at the preliminary design stage of an aircraft wing so that the weak point of the structure can be detected and reinforced accordingly.

During a flight, a crack is prone to propagate further and worsen the aircraft performance. Chapter 3 is focused to study this concern as to how one can calculate the time growth of a crack and its chances of reaching the critical state. The chapter develops a computational framework to estimate time growth of a crack using the modified Paris-Erdogan model represented by the Foreman equation with the using Monte Carlo simulation to include the effect of uncertainties in the model parameters and stochastic nature of the applied load. To obtain the peak cyclic response of the stress in the thin-walled beam for stochastically defined gust loading, random vibration analysis is performed. The peak response statistics are used in crack growth analysis. This approach is used to calculate the probability of a crack approaching its critical size in a given flight duration as well as the remaining flight time before a crack approaches a limiting value. Although the analysis of crack propagation conducted on a beam structure, such as the one considered in this study, is less accurate than that of a more detailed shell finite element analysis of the wing structure, the proposed procedure being simple consumes a lot less computational resource and thus can be used in quick estimates of an evolving operational condition in realtime for health monitoring of the structure under operation.

Chapter 4 addresses the issue of aero-dynamic stability and divergence of a damaged wing modeled by a thin-walled beam with a crack. The analytical solution developed in Chapter 2 greatly facilitates the calculation of the divergence dynamic pressure. Thus, the formulation of Chapter 2 is specialized to solve for the dynamic pressure at divergence of a wing the internal structure of which is represented by a thin-walled beam. Similar to the standard approaches employed in simple beam theories, both iterative and direct approaches are used with exact analytical solution. The numerical results are obtained for different crack configurations, including the uncertainties in its location and size. The results show some interesting trends in divergence conditions under different damage characteristics. 


\subsection{Concluding Remarks}

Each chapter presents numerical results pertaining to the topic of that chapter including its findings. The overall focus of this study has been to use an analytical approach to study the performance of a damaged wing by modeling it by a thin-walled beam primarily because such modeling and analyses are much less computationally demanding and are hoped to capture general features of the more practical problem. Thus, this modeling and analytical framework developed herein is expected to be useful for evaluating the effect of discrete source damage on the static and dynamic responses, crack growth and aeroelastic divergence characteristics of an aircraft wing.

\subsection{Suggestions for Future Research}

The following are suggested as the possible topics for future research.

a. In this study of thin-walled beam with a crack, some simplifying assumptions were made about the crack configurations. In particular, the crack was assumed to be parallel to the longitudinal axis. The crack, however, can develop in arbitrary directions. Future studies could focus on analytical modeling and solution of thin-walled beams with such general crack configurations.

b. The probabilistic analysis of the divergence instabilities was primarily performed using the Monte Carlo studies. In this analysis, the calculation of the probability of the divergence dynamic pressure falling below a limiting value under randomly defined crack conditions was performed using the Monte Carlo simulation approach and was thus observed to be computationally demanding. The implementation of a first order safety index approach to calculate such probabilities for the divergence dynamic pressure defined through implicit conditions appears to be a challenging problem and thus appears to be a good topic for future studies. 


\section{Appendix}




\section{Appendix A Numerical Examples of Jordan Canonical Form}

In this appendix, the details of the vectors and matrices used in the Jordan canonical form solution of the governing equation are provided.

\section{Configurations of system}

Section shape: Rectangular thin-walled

Section dimension (width $\mathrm{x}$ height $\mathrm{x}$ thickness): $0.4 \mathrm{~m} \times 0.2 \mathrm{~m} \times 0.01 \mathrm{~m}$

Material properties: $E=71.7 \times 10^{9} \mathrm{~Pa}$ and $v=0.33$.

Loading type: Uniformly distributed twisting moment

Loading magnitude: $16.67 \mathrm{~N} / \mathrm{m}$

Since the cross-section of the cracked beam in our case has one-fold symmetry, only 4-coupled of the six equations of equilibrium are coupled. For this case, the state-variable and force vectors are defined as follows:

$$
\begin{aligned}
\underline{X} & =\left[\begin{array}{llllllll}
u & u_{1} & \theta_{y} & \theta_{y 1} & \theta_{z} & \theta_{z 1} & \emptyset & \emptyset_{1}
\end{array}\right]^{T} \\
\underline{F} & =\left[\begin{array}{lllllllll}
0 & p_{x} & 0 & m_{x} & 0 & m_{z} & 0 & -m_{\omega}
\end{array}\right]^{T}
\end{aligned}
$$

Whereas the state and other matrices in the state equations are defined as follows for the cracked and uncracked portions of the beam:

\section{Uncracked portion}

$$
\underline{A}=\left[\begin{array}{cccccccc}
1 & 0 & 0 & 0 & 0 & 0 & 0 & 0 \\
0 & -2.16 \times 10^{8} & 0 & 0 & 0 & 0 & 0 & 0 \\
0 & 0 & 1 & 0 & 0 & 0 & 0 & 0 \\
0 & 0 & 0 & -1.91 \times 10^{7} & 0 & 0 & 0 & 0 \\
0 & 0 & 0 & 0 & 1 & 0 & 0 & 0 \\
0 & 0 & 0 & 0 & 0 & -6.47 \times 10^{6} & 0 & 0 \\
0 & 0 & 0 & 0 & 0 & 0 & 1 & 0 \\
0 & 0 & 0 & 0 & 0 & 0 & 0 & -1.27 \times 10^{4}
\end{array}\right]
$$




$$
\begin{gathered}
\underline{B}=\left[\begin{array}{cccccccc}
0 & 1 & 0 & 0 & 0 & 0 & 0 & 0 \\
0 & 0 & 0 & -2.16 \times 10^{8} & 0 & 0 & 0 & 0 \\
0 & 0 & 0 & 1 & 0 & 0 & 0 & 0 \\
0 & 2.16 \times 10^{8}-2.16 \times 10^{8} & 0 & 0 & 0 & 0 & 0 \\
0 & 0 & 0 & 0 & 0 & 1 & 0 & 0 \\
0 & 0 & 0 & 0 & 0 & 0 & 0 & -7.19 \times 10^{5} \\
0 & 0 & 0 & 0 & 0 & 0 & 0 & 1 \\
0 & 0 & 0 & 0 & 0 & 7.19 \times 10^{5}-7.19 \times 10^{5} & 0
\end{array}\right] \\
\underline{F}=\left[\begin{array}{llllllll}
0 & 0 & 0 & 0 & 0 & 16.67 & 0 & 0
\end{array}\right]^{T}
\end{gathered}
$$

Correspondingly, the Jordan matrix, $\underline{J}$, the matrix of transformation, $\underline{\Phi}$, and the matrix of functions, $M(z)$, can be determined following Weintraub [132]. A built-in MATLAB function called "jordan" has been used to facilitate these calculations. Derivations and comprehensive details can be found in the text by Weintraub [132]. The calculated matrices are defined as follows:

$$
\begin{gathered}
J=\left[\begin{array}{cccccccc}
0 & 1 & 0 & 0 & 0 & 0 & 0 & 0 \\
0 & 0 & 1 & 0 & 0 & 0 & 0 & 0 \\
0 & 0 & 0 & 1 & 0 & 0 & 0 & 0 \\
0 & 0 & 0 & 0 & 0 & 0 & 0 & 0 \\
0 & 0 & 0 & 0 & 7.08 & 0 & 0 & 0 \\
0 & 0 & 0 & 0 & 0 & -7.08 & 0 & 0 \\
0 & 0 & 0 & 0 & 0 & 0 & 0 & 1 \\
0 & 0 & 0 & 0 & 0 & 0 & 0 & 0
\end{array}\right] \\
\Phi=\left[\begin{array}{cccccccc}
-11.28 & 0 & 1 & 0 & 0 & 0 & 0.23 & 0 \\
0 & -11.28 & 0 & 1 & 0 & 0 & 0 & 0.23 \\
0 & -11.28 & 0 & 0 & 0 & 0 & 0 & 0.23 \\
0 & 0 & -11.28 & 0 & 0 & 0 & 0 & 0 \\
0 & 0 & 1.13 & 0 & -0.01 & 0.01 & 1.13 & 0 \\
0 & 0 & 1 & 1.13-0.06-0.06 & 0 & 1.13 \\
0 & 0 & 0 & 1.13-0.56-0.56 & 0 & 1.13 \\
0 & 0 & 0 & 0 & -3.98 & 3.98 & 0 & 0
\end{array}\right]
\end{gathered}
$$




$$
\underline{M(z)}=\left[\begin{array}{cccccccc}
1 & z & \frac{z^{2}}{2} & \frac{z^{3}}{6} & 0 & 0 & 0 & 0 \\
0 & 1 & z & \frac{z^{2}}{2} & 0 & 0 & 0 & 0 \\
0 & 0 & 1 & z & 0 & 0 & 0 & 0 \\
0 & 0 & 0 & 1 & 0 & 0 & 0 & 0 \\
0 & 0 & 0 & 0 & \exp (7.08) & 0 & 0 & 0 \\
0 & 0 & 0 & 0 & 0 & \exp (-7.08) & 0 & 0 \\
0 & 0 & 0 & 0 & 0 & 0 & 1 & z \\
0 & 0 & 0 & 0 & 0 & 0 & 0 & 1
\end{array}\right]
$$

\section{Cracked portion}

$$
\begin{aligned}
& \underline{A}=\left[\begin{array}{cccccccc}
1 & 0 & 0 & 0 & 0 & 0 & 0 & 0 \\
0 & -2.16 \times 10^{8} & 0 & 0 & 0 & 0 & 0 & 0 \\
0 & 0 & 1 & 0 & 0 & 0 & 0 & 0 \\
0 & 0 & 0 & -1.91 \times 10^{7} & 0 & 0 & 0 & -4.59 \times 10^{6} \\
0 & 0 & 0 & 0 & 1 & 0 & 0 & 0 \\
0 & 0 & 0 & 0 & 0 & -6.47 \times 10^{6} & 0 & 0 \\
0 & 0 & 0 & 0 & 0 & 0 & 1 & 0 \\
0 & 0 & 0 & -4.59 \times 10^{6} & 0 & 0 & 0 & -1.95 \times 10^{6}
\end{array}\right] \\
& \underline{B}=\left[\begin{array}{cccccccc}
0 & 1 & 0 & 0 & 0 & 0 & 0 & 0 \\
0 & 0 & 0 & -2.16 \times 10^{8} & 0 & 0 & 0 & 0 \\
0 & 0 & 0 & 1 & 0 & 0 & 0 & 0 \\
0 & 2.16 \times 10^{8}-2.16 \times 10^{8} & 0 & 0 & 0 & 0 & 0 \\
0 & 0 & 0 & 0 & 0 & 1 & 0 & 0 \\
0 & 0 & 0 & 0 & 0 & 0 & 0 & -6.47 \times 10^{6} \\
0 & 0 & 0 & 0 & 0 & 0 & 0 & 1 \\
0 & 0 & 0 & 0 & 0 & 6.47 \times 10^{6}-6.47 \times 10^{6} & 0
\end{array}\right] \\
& \underline{F}=\left\lfloor\begin{array}{llllllll}
0 & 0 & 0 & 0 & 0 & 16.67 & 0 & 0
\end{array}\right]^{T}
\end{aligned}
$$

The corresponding Jordan matrix, $\underline{J}$, matrix of transformation, $\underline{\Phi}$, and matrix of functions, $\underline{M(z)}$, for this case are as follows: 


$$
\begin{aligned}
& \underline{J}=\left[\begin{array}{cccccccc}
0 & 1 & 0 & 0 & 0 & 0 & 0 & 0 \\
0 & 0 & 1 & 0 & 0 & 0 & 0 & 0 \\
0 & 0 & 0 & 1 & 0 & 0 & 0 & 0 \\
0 & 0 & 0 & 0 & 0 & 0 & 0 & 0 \\
0 & 0 & 0 & 0 & -0.11 & 0 & 0 & 0 \\
0 & 0 & 0 & 0 & 0 & 0.11 & 0 & 0 \\
0 & 0 & 0 & 0 & 0 & 0 & 0 & 1 \\
0 & 0 & 0 & 0 & 0 & 0 & 0 & 0
\end{array}\right] \\
& \Phi=\left[\begin{array}{cccccccc}
-11.28 & 0 & 1,409 & 0 & 5,114 & -5,114 & 255 & 0 \\
0 & -11.28 & 0 & 1,409 & -576 & -576 & 0 & 255 \\
0 & -11.28 & 0 & 1,408 & -576 & -576 & 0 & 255 \\
0 & 0 & -11.28 & 0 & 64.92 & -64.92 & 0 & 0 \\
0 & 0 & -5,398 & 0 & -21,292 & 21,292 & -600 & 0 \\
0 & 0 & 0 & -5,398 & 2,399 & 2,399 & 0 & -600 \\
0 & 0 & 0 & -5,402 & 2,401 & 2401 & 0 & -600 \\
0 & 0 & 0 & 0 & -271 & 271 & 0 & 0
\end{array}\right] \\
& \underline{M(z)}=\left[\begin{array}{cccccccc}
1 & z & \frac{z^{2}}{2} & \frac{z^{3}}{6} & 0 & 0 & 0 & 0 \\
0 & 1 & z & \frac{z^{2}}{2} & 0 & 0 & 0 & 0 \\
0 & 0 & 1 & z & 0 & 0 & 0 & 0 \\
0 & 0 & 0 & 1 & 0 & 0 & 0 & 0 \\
0 & 0 & 0 & 0 & \exp (-0.11) & 0 & 0 & 0 \\
0 & 0 & 0 & 0 & 0 & \exp (0.11) & 0 & 0 \\
0 & 0 & 0 & 0 & 0 & 0 & 1 & z \\
0 & 0 & 0 & 0 & 0 & 0 & 0 & 1
\end{array}\right]
\end{aligned}
$$




\section{Appendix B Assembled Matrix Components}

\section{$\underline{\text { Matrix } G}$}

\section{Column 1-12}

$$
\begin{aligned}
& G(1,1: 12)=\underline{\Phi}_{1}^{(1)} \underline{M(0)}^{(1)} \\
& G(2,1: 12)=\underline{\Phi}_{3}^{(1)} \underline{M(0)}^{(1)} \\
& G(3,1: 12)=\underline{\Phi}_{5}^{(1)} M(0)^{(1)} \\
& G(4,1: 12)=\underline{\Phi}_{7}^{(1)} M(0)^{(1)} \\
& G(5,1: 12)=\underline{\Phi}_{9}^{(1)} \underline{M(0)}^{(1)} \\
& G(6,1: 12)=\underline{\Phi}_{11}^{(1)} \underline{M(0)}^{(1)} \\
& G(7,1: 12)=\underline{\Phi}_{1}^{(1)}{\underline{M\left(l_{1}\right)^{(1)}}}^{(1)} \\
& G(8,1: 12)=\underline{\Phi}_{3}^{(1)}{\underline{M\left(l_{1}\right)^{(1)}}}^{(1)} \\
& G(9,1: 12)=\left[\underline{\Phi}_{5}^{(1)}+d_{2} \underline{\Phi}_{11}^{(1)}\right] \underline{M\left(l_{1}\right)^{(1)}} \\
& G(10,1: 12)=\left[\underline{\Phi}_{7}^{(1)}-d_{3} \underline{\Phi}_{11}^{(1)}\right]{\underline{M\left(l_{1}\right)}}^{(1)} \\
& G(11,1: 12)=\underline{\Phi}_{9}^{(1)}{\underline{M\left(l_{1}\right)}}^{(1)} \\
& G(12,1: 12)=d_{4} \underline{\Phi}_{11}^{(1)}{\underline{M\left(l_{1}\right)^{(1)}}}^{(1)} \\
& G(13,1: 12)=\left[G J_{\theta y}^{(1)} \underline{\Phi}_{10}^{(1)}-G Q_{y z}^{(1)} \underline{\Phi}_{11}^{(1)}+G Q_{x y}^{(1)}\left(\underline{\Phi}_{4}^{(1)}+\underline{\Phi}_{5}^{(1)}\right)+G Q_{y y}^{(1)}\left(\underline{\Phi}_{2}^{(1)}-\underline{\Phi}_{7}^{(1)}\right)\right]{\underline{M\left(l_{1}\right)}}^{(1)} \\
& G(14,1: 12)=\left[G J_{\theta x}^{(1)} \underline{\Phi}_{10}^{(1)}-G Q_{x z}^{(1)} \underline{\Phi}_{11}^{(1)}+G Q_{x x}^{(1)}\left(\underline{\Phi}_{4}^{(1)}+\underline{\Phi}_{5}^{(1)}\right)+G Q_{x y}^{(1)}\left(\underline{\Phi}_{2}^{(1)}-\underline{\Phi}_{7}^{(1)}\right)\right]{\underline{M\left(l_{1}\right)}}^{(1)} \\
& G(15,1: 12)=\left[-E \Gamma_{z x}^{(1)} \underline{\Phi}_{12}^{(1)}+E I_{x x}^{(1)} \underline{\Phi}_{6}^{(1)}-E I_{x y}^{(1)} \underline{\Phi}_{8}^{(1)}\right] \underline{M\left(l_{1}\right)^{(1)}} \\
& G(16,1: 12)=\left[E \Gamma_{z y}^{(1)} \underline{\Phi}_{12}^{(1)}+E I_{x y}^{(1)} \underline{\Phi}_{6}^{(1)}-E I_{y y}^{(1)} \underline{\Phi}_{0}^{(1)}\right] M\left(l_{1}\right)^{(1)} \\
& G(17,1: 12)=\left[G J_{\theta \theta}^{(1)} \underline{\Phi}_{10}^{(1)}-G J_{\theta z}^{(1)} \underline{\Phi}_{11}^{(1)}+G J_{\theta x}^{(1)}\left(\underline{\Phi}_{4}^{(1)}+\underline{\Phi}_{5}^{(1)}\right)+G J_{\theta y}^{(1)}\left(\underline{\Phi}_{2}^{(1)}-\underline{\Phi}_{7}^{(1)}\right)\right] M\left(l_{1}\right)^{(1)} \\
& G(18,1: 12)=\left[E \Gamma_{z z}^{(1)} \underline{\Phi}_{12}^{(1)}-E \Gamma_{z x}^{(1)} \underline{\Phi}_{6}^{(1)}-E \Gamma_{z y}^{(2)} \underline{\Phi}_{8}^{(1)}\right] M\left(l_{1}\right)^{(1)}
\end{aligned}
$$

\section{Column 13-24}

$G(7,13: 24)=-\underline{\Phi}_{1}^{(2)} \underline{M(0)^{(2)}}$ 


$$
\begin{aligned}
& G(8,13: 24)=-\underline{\Phi}_{3}^{(2)} \underline{M(0)}^{(2)} \\
& G(9,13: 24)=-\underline{\Phi}_{5}^{(2)} \underline{M(0)}^{(2)} \\
& G(10,13: 24)=-\underline{\Phi}_{7}^{(2)} \underline{M(0)}^{(2)} \\
& G(11,13: 24)=-\underline{\Phi}_{9}^{(2)} M(0)^{(2)} \\
& G(12,13: 24)=-\underline{\Phi}_{11}^{(2)} \underline{M(0)^{(2)}} \\
& G(13,13: 24)=-\left[G J_{\theta y}^{(2)} \Phi_{10}^{(2)}-G Q_{y z}^{(2)} \Phi_{11}^{(2)}+G Q_{x y}^{(2)}\left(\underline{\Phi}_{4}^{(2)}+\underline{\Phi}_{5}^{(2)}\right)\right. \\
& \left.+G Q_{y y}^{(2)}\left(\underline{\Phi}_{2}^{(2)}-\underline{\Phi}_{7}^{(2)}\right)\right] M(0)^{(2)} \\
& G(14,13: 24)=-\left[G J_{\theta x}^{(2)} \Phi_{10}^{(2)}-G Q_{x z}^{(2)} \Phi_{11}^{(2)}+G Q_{x x}^{(2)}\left(\underline{\Phi}_{4}^{(2)}+\underline{\Phi}_{5}^{(2)}\right)\right. \\
& \left.+G Q_{x y}^{(2)}\left(\underline{\Phi}_{2}^{(2)}-\underline{\Phi}_{7}^{(2)}\right)\right] M(0)^{(2)} \\
& G(15,13: 24)=-\left[-E \Gamma_{z x}^{(2)} \underline{\Phi}_{12}^{(2)}+E I_{x x}^{(2)} \underline{\Phi}_{6}^{(2)}-E I_{x y}^{(2)} \underline{\Phi}_{8}^{(2)}\right] M(0)^{(2)} \\
& G(16,13: 24)=-\left[E \Gamma_{z y}^{(2)} \underline{\Phi}_{12}^{(2)}+E I_{x y}^{(2)} \underline{\Phi}_{6}^{(2)}-E I_{y y}^{(2)} \underline{\Phi}_{8}^{(2)}\right] \underline{M(0)^{(2)}} \\
& G(17,13: 24)=-\left[G J_{\theta \theta}^{(2)} \underline{\Phi}_{10}^{(2)}-G J_{\theta z}^{(2)} \underline{\Phi}_{11}^{(2)}+G J_{\theta x}^{(2)}\left(\underline{\Phi}_{4}^{(2)}+\underline{\Phi}_{5}^{(2)}\right)\right. \\
& \left.+G J_{\theta y}^{(2)}\left(\underline{\Phi}_{2}^{(2)}-\underline{\Phi}_{7}^{(2)}\right)\right] M(0)^{(2)} \\
& G(18,13: 24)=-\left\langle d_{2}\left[-E \Gamma_{z x}^{(2)} \underline{\Phi}_{12}^{(2)}+E I_{x x}^{(2)} \underline{\Phi}_{6}^{(2)}-E I_{x y}^{(2)} \underline{\Phi}_{8}^{(2)}\right]\right. \\
& +d_{3}\left[E \Gamma_{z y}^{(2)} \underline{\Phi}_{12}^{(2)}+E I_{x y}^{(2)} \Phi_{6}^{(2)}\right. \\
& \left.\left.-E I_{y y}^{(2)}\right]-d_{4}\left[E \Gamma_{z z}^{(2)} \underline{\Phi}_{12}^{(2)}-E \Gamma_{z x}^{(2)} \underline{\Phi}_{6}^{(2)}-E \Gamma_{z y}^{(2)} \underline{\Phi}_{8}^{(2)}\right]\right\rangle \underline{M(0)^{(2)}} \\
& G(19,13: 24)=\underline{\Phi}_{1}^{(2)}{\underline{M\left(l_{2}\right)}}^{(2)} \\
& G(20,13: 24)=\underline{\Phi}_{3}^{(2)} \underline{M\left(l_{2}\right)^{(2)}} \\
& G(21,13: 24)=\left[\underline{\Phi}_{5}^{(1)}+d_{2} \underline{\Phi}_{11}^{(1)}\right] \underline{M\left(l_{2}\right)^{(2)}} \\
& G(22,13: 24)=\left[\underline{\Phi}_{7}^{(1)}-d_{3} \underline{\Phi}_{11}^{(1)}\right]{\underline{M\left(l_{2}\right)}}^{(2)} \\
& G(23,13: 24)=\underline{\Phi}_{9}^{(2)} \underline{M\left(l_{2}\right)^{(2)}} \\
& G(24,13: 24)=d_{4} \underline{\Phi}_{11}^{(2)} \underline{M\left(l_{2}\right)^{(2)}}
\end{aligned}
$$




$$
\begin{aligned}
& G(25,13: 24)=\left[G J_{\theta y}^{(2)} \underline{\Phi}_{10}^{(2)}-G Q_{y z}^{(2)} \underline{\Phi}_{11}^{(2)}+G Q_{x y}^{(2)}\left(\underline{\Phi}_{4}^{(2)}+\underline{\Phi}_{5}^{(2)}\right)\right. \\
& \left.+G Q_{y y}^{(2)}\left(\underline{\Phi}_{2}^{(2)}-\underline{\Phi}_{7}^{(2)}\right)\right]{\underline{M\left(l_{2}\right)^{(2)}}}^{(2)} \\
& G(26,13: 24)=\left[G J_{\theta x}^{(2)} \underline{\Phi}_{10}^{(2)}-G Q_{x z}^{(2)} \underline{\Phi}_{11}^{(2)}+G Q_{x x}^{(2)}\left(\underline{\Phi}_{4}^{(2)}+\underline{\Phi}_{5}^{(2)}\right)\right. \\
& \left.+G Q_{x y}^{(2)}\left(\underline{\Phi}_{2}^{(2)}-\underline{\Phi}_{7}^{(2)}\right)\right]{\underline{M\left(l_{2}\right)^{(2)}}}^{(2)} \\
& G(27,13: 24)=\left[-E \Gamma_{z x}^{(2)} \underline{\Phi}_{12}^{(2)}+E I_{x x}^{(2)} \underline{\Phi}_{6}^{(2)}-E I_{x y}^{(2)} \underline{\Phi}_{8}^{(2)}\right]{\underline{M\left(l_{2}\right)}}^{(2)} \\
& G(28,13: 24)=\left[E \Gamma_{z y}^{(2)} \underline{\Phi}_{12}^{(2)}+E I_{x y}^{(2)} \underline{\Phi}_{6}^{(2)}-E I_{y y}^{(2)} \underline{\Phi}_{8}^{(2)}\right] \underline{M\left(l_{2}\right)^{(2)}} \\
& G(29,13: 24)=\left[G J_{\theta \theta}^{(2)} \underline{\Phi}_{10}^{(2)}-G J_{\theta z}^{(2)} \underline{\Phi}_{11}^{(2)}+G J_{\theta x}^{(2)}\left(\underline{\Phi}_{4}^{(2)}+\underline{\Phi}_{5}^{(2)}\right)+G J_{\theta y}^{(2)}\left(\underline{\Phi}_{2}^{(2)}-\underline{\Phi}_{7}^{(2)}\right)\right]{\underline{M\left(l_{2}\right)}}^{(2)} \\
& G(30,13: 24)=\left[E \Gamma_{z z}^{(2)} \underline{\Phi}_{12}^{(2)}-E \Gamma_{z x}^{(2)} \underline{\Phi}_{6}^{(2)}-E \Gamma_{z y}^{(2)} \underline{\Phi}_{8}^{(2)}\right]{\underline{M\left(l_{2}\right)}}^{(2)}
\end{aligned}
$$

\section{Column 25-36}

$$
\begin{aligned}
& G(19,25: 36)=\underline{\Phi}_{1}^{(3)} M(0)^{(3)} \\
& G(20,25: 36)=\underline{\Phi}_{3}^{(3)} \underline{M(0)^{(3)}} \\
& G(21,25: 36)=\underline{\Phi}_{5}^{(3)} \underline{M(0)}^{(3)} \\
& G(22,25: 36)=\underline{\Phi}_{7}^{(3)} \underline{M(0)^{(3)}} \\
& G(23,25: 36)=\underline{\Phi}_{9}^{(3)} \underline{M(0)^{(3)}} \\
& G(24,25: 36)=\underline{\Phi}_{11}^{(3)} \underline{M(0)}^{(3)} \\
& G(25,25: 36)=-\left[G J_{\theta y}^{(3)} \Phi_{10}^{(3)}-G Q_{y z}^{(3)} \Phi_{11}^{(3)}+G Q_{x y}^{(3)}\left(\underline{\Phi}_{4}^{(3)}+\underline{\Phi}_{5}^{(3)}\right)\right. \\
& \left.+G Q_{y y}^{(3)}\left(\underline{\Phi}_{2}^{(3)}-\underline{\Phi}_{7}^{(3)}\right)\right] M(0)^{(3)} \\
& G(26,25: 36)=-\left[G J_{\theta x}^{(3)} \Phi_{10}^{(3)}-G Q_{x z}^{(3)} \underline{\Phi}_{11}^{(3)}+G Q_{x x}^{(3)}\left(\underline{\Phi}_{4}^{(3)}+\underline{\Phi}_{5}^{(3)}\right)\right. \\
& \left.+G Q_{x y}^{(3)}\left(\underline{\Phi}_{2}^{(3)}-\underline{\Phi}_{7}^{(3)}\right)\right] M(0)^{(3)} \\
& \left.G(27,25: 36)=-E \Gamma_{z x}^{(3)} \underline{\Phi}_{12}^{(3)}+E I_{x x}^{(3)} \underline{\Phi}_{6}^{(3)}-E I_{x y}^{(3)} \underline{\Phi}_{8}^{(3)}\right] \underline{M(0)}{ }^{(3)} \\
& G(28,25: 36)=-\left[E \Gamma_{z y}^{(3)} \underline{\Phi}_{12}^{(3)}+E I_{x y}^{(3)} \underline{\Phi}_{6}^{(3)}-E I_{y y}^{(3)} \underline{\Phi}_{8}^{(3)}\right] \underline{M(0)}{ }^{(3)} \\
& G(29,25: 36)=-\left[G J_{\theta \theta}^{(3)} \underline{\Phi}_{10}^{(3)}-G J_{\theta z}^{(3)} \underline{\Phi}_{11}^{(3)}+G J_{\theta x}^{(3)}\left(\underline{\Phi}_{4}^{(3)}+\underline{\Phi}_{5}^{(3)}\right)\right. \\
& \left.+G J_{\theta y}^{(3)}\left(\underline{\Phi}_{2}^{(3)}-\underline{\Phi}_{7}^{(3)}\right)\right] \underline{M(0)}^{(3)}
\end{aligned}
$$




$$
\begin{aligned}
G(30,25: 36)= & -\left\langle d_{2}\left[-E \Gamma_{z x}^{(3)} \underline{\Phi}_{12}^{(3)}+E I_{x x}^{(3)} \underline{\Phi}_{6}^{(3)}-E I_{x y}^{(3)} \underline{\Phi}_{8}^{(3)}\right]\right. \\
& +d_{3}\left[E \Gamma_{z y}^{(3)} \underline{\Phi}_{12}^{(3)}+E I_{x y}^{(3)} \underline{\Phi}_{6}^{(3)}\right. \\
& \left.\left.-E I_{y y}^{(3)}\right]-d_{4}\left[E \Gamma_{z z}^{(3)} \underline{\Phi}_{12}^{(3)}-E \Gamma_{z x}^{(3)} \underline{\Phi}_{6}^{(3)}-E \Gamma_{z y}^{(3)} \underline{\Phi}_{8}^{(3)}\right]\right\rangle \underline{M(0)}^{(3)} \\
G(31,25: 36)= & {\left[G J_{\theta y}^{(3)} \underline{\Phi}_{10}^{(3)}-G Q_{y z}^{(3)} \underline{\Phi}_{11}^{(3)}+G Q_{x y}^{(3)}\left(\underline{\Phi}_{4}^{(3)}+\underline{\Phi}_{5}^{(3)}\right)\right.} \\
& \left.+G Q_{y y}^{(3)}\left(\underline{\Phi}_{2}^{(3)}-\underline{\Phi}_{7}^{(3)}\right)\right] \underline{M\left(l_{3}\right)^{(3)}} \\
G(32,25: 36)= & {\left[G J_{\theta x}^{(3)} \underline{\Phi}_{10}^{(3)}-G Q_{x z}^{(3)} \underline{\Phi}_{11}^{(3)}+G Q_{x x}^{(3)}\left(\underline{\Phi}_{4}^{(3)}+\underline{\Phi}_{5}^{(3)}\right)\right.} \\
& \left.+G Q_{x y}^{(3)}\left(\underline{\Phi}_{2}^{(3)}-\underline{\Phi}_{7}^{(3)}\right)\right]{\underline{M\left(l_{3}\right)^{(3)}}}^{(3)} \\
G(33,25: 36)= & {\left[-E \Gamma_{z x}^{(3)} \underline{\Phi}_{12}^{(3)}+E I_{x x}^{(3)} \underline{\Phi}_{6}^{(3)}-E I_{x y}^{(3)} \underline{\Phi}_{8}^{(3)}\right] \underline{M\left(l_{3}\right)^{(3)}} } \\
G(34,25: 36)= & {\left[E \Gamma_{z y}^{(3)} \underline{\Phi}_{12}^{(3)}+E I_{x y}^{(3)} \underline{\Phi}_{6}^{(3)}-E I_{y y}^{(3)} \underline{\Phi}_{8}^{(3)}\right] M\left(l_{3}\right)^{(3)} } \\
G(35,25: 36)= & {\left[G J_{\theta \theta}^{(3)} \underline{\Phi}_{10}^{(3)}-G J_{\theta z}^{(3)} \underline{\Phi}_{11}^{(3)}+G J_{\theta x}^{(3)}\left(\underline{\Phi}_{4}^{(3)}+\underline{\Phi}_{5}^{(3)}\right)+G J_{\theta y}^{(3)}\left(\underline{\Phi}_{2}^{(3)}-\underline{\Phi}_{7}^{(3)}\right)\right] \underline{M\left(l_{3}\right)^{(3)}} } \\
G(36,25: 36)= & {\left[E \Gamma_{z z}^{(3)} \underline{\Phi}_{12}^{(3)}-E \Gamma_{z x}^{(3)} \underline{\Phi}_{6}^{(3)}-E \Gamma_{z y}^{(3)} \underline{\Phi}_{8}^{(3)}\right] M\left(l_{3}\right)^{(3)} }
\end{aligned}
$$

\section{Vector c}

Row 1-12

$c(1: 12,1)=\left[\begin{array}{lllllllllllll}c_{1}^{(1)} & c_{2}^{(1)} & c_{3}^{(1)} & c_{4}^{(1)} & c_{5}^{(1)} & c_{6}^{(1)} & c_{7}^{(1)} & c_{8}^{(1)} & c_{9}^{(1)} & c_{10}^{(1)} & c_{11}^{(1)} & c_{12}^{(1)}\end{array}\right]^{T}$

\section{Row 13-24}

$c(13: 24,1)=\left[\begin{array}{llllllllllll}c_{1}^{(2)} & c_{2}^{(2)} & c_{3}^{(2)} & c_{4}^{(2)} & c_{5}^{(2)} & c_{6}^{(2)} & c_{7}^{(2)} & c_{8}^{(2)} & c_{9}^{(2)} & c_{10}^{(2)} & c_{11}^{(2)} & c_{12}^{(2)}\end{array}\right]^{T}$

Row 25-36

$c(25: 36,1)=\left[\begin{array}{llllllllllll}c_{1}^{(3)} & c_{2}^{(3)} & c_{3}^{(3)} & c_{4}^{(3)} & c_{5}^{(3)} & c_{6}^{(3)} & c_{7}^{(3)} & c_{8}^{(3)} & c_{9}^{(3)} & c_{10}^{(3)} & c_{11}^{(3)} & c_{12}^{(3)}\end{array}\right]^{T}$

\section{Vector $F_{G}$, Row 1-12}

$F_{G}(1,1)=-\underline{\Phi}_{1}^{(1)} \underline{I(0)}^{(1)}$

$F_{G}(2,1)=-\underline{\Phi}_{3}^{(1)} \underline{I(0)}^{(1)}$

$F_{G}(3,1)=-\underline{\Phi}_{5}^{(1)} \underline{I(0)}^{(1)}$

$F_{G}(4,1)=-\underline{\Phi}_{7}^{(1)} \underline{I(0)}^{(1)}$ 


$$
\begin{aligned}
& F_{G}(5,1)=-\underline{\Phi}_{9}^{(1)} \underline{I(0)}^{(1)} \\
& F_{G}(6,1)=-\underline{\Phi}_{11}^{(1)} \underline{I(0)}^{(1)} \\
& F_{G}(7,1)=\underline{\Phi}_{1}^{(2)} \underline{I(0)}{ }^{(2)}-\underline{\Phi}_{1}^{(1)}{\underline{I\left(l_{1}\right)^{(1)}}}^{(1)} \\
& F_{G}(8,1)=\underline{\Phi}_{3}^{(2)} \underline{I(0)}^{(2)}-\underline{\Phi}_{3}^{(1)}{\underline{I\left(l_{1}\right)^{(1)}}}^{(1)} \\
& F_{G}(9,1)=\underline{\Phi}_{5}^{(2)} \underline{I(0)}^{(2)}-\left[\underline{\Phi}_{5}^{(1)}+d_{2} \underline{\Phi}_{11}^{(1)}\right] \underline{I\left(l_{1}\right)}{ }^{(1)} \\
& F_{G}(10,1)=\underline{\Phi}_{7}^{(2)} \underline{I(0)}^{(2)}-\left[\underline{\Phi}_{7}^{(1)}-d_{3} \underline{\Phi}_{11}^{(1)}\right]{\underline{I\left(l_{1}\right)^{(1)}}}^{(1)} \\
& F_{G}(11,1)=\underline{\Phi}_{9}^{(2)} \underline{I(0)}^{(2)}-\underline{\Phi}_{9}^{(1)}{\underline{I\left(l_{1}\right)}}^{(1)} \\
& F_{G}(12,1)=\underline{\Phi}_{11}^{(2)} \underline{I(0)}^{(2)}-d_{4} \underline{\Phi}_{11}^{(1)}{\underline{I\left(l_{1}\right)}}^{(1)}
\end{aligned}
$$

\section{Vector $F_{G}$, Row 13-24}

$$
\begin{aligned}
F_{G}(13,1)=\left[G J_{\theta y}^{(2)} \underline{\Phi}_{10}^{(2)}-G Q_{y z}^{(2)} \underline{\Phi}_{11}^{(2)}+G Q_{x y}^{(2)}\left(\underline{\Phi}_{4}^{(2)}+\underline{\Phi}_{5}^{(2)}\right)+G Q_{y y}^{(2)}\left(\underline{\Phi}_{2}^{(2)}\right.\right. & \\
& \left.\left.-\underline{\Phi}_{7}^{(2)}\right)\right] \underline{I(0)}{ }^{(2)}-\left[G J_{\theta y}^{(1)} \underline{\Phi}_{10}^{(1)}-G Q_{y z}^{(1)} \underline{\Phi}_{11}^{(1)}+G Q_{x y}^{(1)}\left(\underline{\Phi}_{4}^{(1)}+\underline{\Phi}_{5}^{(1)}\right)+G Q_{y y}^{(1)}\left(\underline{\Phi}_{2}^{(1)}\right.\right. \\
& \left.\left.-\underline{\Phi}_{7}^{(1)}\right)\right] \underline{I\left(l_{1}\right)^{(1)}} \\
F_{G}(14,1)=[ & \left.G J_{\theta x}^{(2)} \underline{\Phi}_{10}^{(2)}-G Q_{x z}^{(2)} \underline{\Phi}_{11}^{(2)}+G Q_{x x}^{(2)}\left(\underline{\Phi}_{4}^{(2)}+\underline{\Phi}_{5}^{(2)}\right)+G Q_{x y}^{(2)}\left(\underline{\Phi}_{2}^{(2)}-\underline{\Phi}_{7}^{(2)}\right)\right] \underline{I(0)}^{(2)} \\
& -\left[G J_{\theta x}^{(1)} \underline{\Phi}_{10}^{(1)}-G Q_{x z}^{(1)} \underline{\Phi}_{11}^{(1)}+G Q_{x x}^{(1)}\left(\underline{\Phi}_{4}^{(1)}+\underline{\Phi}_{5}^{(1)}\right)+G Q_{x y}^{(1)}\left(\underline{\Phi}_{2}^{(1)}-\underline{\Phi}_{7}^{(1)}\right)\right] \underline{I\left(l_{1}\right)^{(1)}} \\
F_{G}(15,1)=[ & \left.-E \Gamma_{z x}^{(2)} \underline{\Phi}_{12}^{(2)}+E I_{x x}^{(2)} \underline{\Phi}_{6}^{(2)}-E I_{x y}^{(2)} \underline{\Phi}_{8}^{(2)}\right] \underline{I(0)}^{(2)}-E \Gamma_{z x}^{(1)} \underline{\Phi}_{12}^{(1)}+E I_{x x}^{(1)} \underline{\Phi}_{6}^{(1)} \\
& \left.-E I_{x y}^{(1)} \underline{\Phi}_{8}^{(1)}\right] \underline{I\left(l_{1}\right)^{(1)}} \\
F_{G}(16,1)=[ & E \Gamma_{z y}^{(2)} \underline{\Phi}_{12}^{(2)}+E I_{x y}^{(2)} \underline{\Phi}_{6}^{(2)} \\
& \left.-E I_{y y}^{(2)} \underline{\Phi}_{8}^{(2)}\right] \underline{I(0)}^{(2)}-\left[E \Gamma_{z y}^{(1)} \underline{\Phi}_{12}^{(1)}+E I_{x y}^{(1)} \underline{\Phi}_{6}^{(1)}-E I_{y y}^{(1)} \underline{\Phi}_{8}^{(1)}\right]{\underline{I\left(l_{1}\right)}}^{(1)} \\
F_{G}(17,1)=[ & \left.G J_{\theta \theta}^{(2)} \underline{\Phi}_{10}^{(2)}-G J_{\theta z}^{(2)} \underline{\Phi}_{11}^{(2)}+G J_{\theta x}^{(2)}\left(\underline{\Phi}_{4}^{(2)}+\underline{\Phi}_{5}^{(2)}\right)+G J_{\theta y}^{(2)}\left(\underline{\Phi}_{2}^{(2)}-\underline{\Phi}_{7}^{(2)}\right)\right] \underline{I(0)}^{(2)} \\
& -\left[G J_{\theta \theta}^{(1)} \underline{\Phi}_{10}^{(1)}-G J_{\theta z}^{(1)} \underline{\Phi}_{11}^{(1)}+G J_{\theta x}^{(1)}\left(\underline{\Phi}_{4}^{(1)}+\underline{\Phi}_{5}^{(1)}\right)+G J_{\theta y}^{(1)}\left(\underline{\Phi}_{2}^{(1)}-\underline{\Phi}_{7}^{(1)}\right)\right]{\underline{I\left(l_{1}\right)}}^{(1)}
\end{aligned}
$$




$$
\begin{aligned}
& F_{G}(18,1)=\left\langle d_{2}\left[-E \Gamma_{z x}^{(2)} \underline{\Phi}_{12}^{(2)}+E I_{x x}^{(2)} \underline{\Phi}_{6}^{(2)}-E I_{x y}^{(2)} \underline{\Phi}_{8}^{(2)}\right]\right. \\
& +d_{3}\left[E \Gamma_{z y}^{(2)} \underline{\Phi}_{12}^{(2)}+E I_{x y}^{(2)} \underline{\Phi}_{6}^{(2)}\right. \\
& \left.\left.-E I_{y y}^{(2)}\right]-d_{4}\left[E \Gamma_{z z}^{(2)} \underline{\Phi}_{12}^{(2)}-E \Gamma_{z x}^{(2)} \underline{\Phi}_{6}^{(2)}-E \Gamma_{z y}^{(2)} \underline{\Phi}_{8}^{(2)}\right]\right\rangle \underline{I(0)}^{(2)} \\
& -\left[E \Gamma_{z Z}^{(1)} \underline{\Phi}_{12}^{(1)}-E \Gamma_{z x}^{(1)} \underline{\Phi}_{6}^{(1)}-E \Gamma_{z y}^{(2)} \underline{\Phi}_{8}^{(1)}\right]{\underline{I\left(l_{1}\right)^{(1)}}}^{(1)} \\
& F_{G}(19,1)=\underline{\Phi}_{2}^{(3)} I(0)^{(3)}-\underline{\Phi}_{1}^{(2)} I\left(l_{2}\right)^{(2)} \\
& F_{G}(20,1)=\underline{\Phi}_{2}^{(3)} \underline{I(0)}^{(3)}-\underline{\Phi}_{3}^{(2)}{\underline{I\left(l_{2}\right)}}^{(2)} \\
& F_{G}(21,1)=\underline{\Phi}_{3}^{(3)} I(0)^{(3)}-\left[\underline{\Phi}_{5}^{(1)}+d_{2} \underline{\Phi}_{11}^{(1)}\right]{\underline{I\left(l_{2}\right)}}^{(2)} \\
& F_{G}(22,1)=\underline{\Phi}_{5}^{(3)} I(0) \underline{(}^{(3)}-\left[\underline{\Phi}_{7}^{(1)}-d_{3} \underline{\Phi}_{11}^{(1)}\right]{\underline{I\left(l_{2}\right)}}^{(2)} \\
& F_{G}(23,1)=\underline{\Phi}_{7}^{(3)} I(0) \underline{S}^{(3)}-\underline{\Phi}_{9}^{(2)} I\left(l_{2}\right)^{(2)} \\
& F_{G}(24,1)=\underline{\Phi}_{9}^{(3)} \underline{I(0)}^{(3)}-d_{4} \underline{\Phi}_{11}^{(2)}{\underline{I\left(l_{2}\right)}}^{(2)}
\end{aligned}
$$

\section{Vector $F_{G}$, Row 25-36}

$$
\begin{aligned}
& F_{G}(25,1)=\left[G J_{\theta y}^{(3)} \underline{\Phi}_{10}^{(3)}-G Q_{y z}^{(3)} \underline{\Phi}_{11}^{(3)}+G Q_{x y}^{(3)}\left(\underline{\Phi}_{4}^{(3)}+\underline{\Phi}_{5}^{(3)}\right)+G Q_{y y}^{(3)}\left(\underline{\Phi}_{2}^{(3)}-\underline{\Phi}_{7}^{(3)}\right)\right] \underline{I(0)}^{(3)} \\
& -\left[G J_{\theta y}^{(2)} \underline{\Phi}_{10}^{(2)}-G Q_{y z}^{(2)} \underline{\Phi}_{11}^{(2)}+G Q_{x y}^{(2)}\left(\underline{\Phi}_{4}^{(2)}+\underline{\Phi}_{5}^{(2)}\right)\right. \\
& \left.+G Q_{y y}^{(2)}\left(\underline{\Phi}_{2}^{(2)}-\underline{\Phi}_{7}^{(2)}\right)\right]{\underline{I\left(l_{2}\right)}}^{(2)} \\
& F_{G}(26,1)=\left[G J_{\theta x}^{(3)} \underline{\Phi}_{10}^{(3)}-G Q_{x z}^{(3)} \underline{\Phi}_{11}^{(3)}+G Q_{x x}^{(3)}\left(\underline{\Phi}_{4}^{(3)}+\underline{\Phi}_{5}^{(3)}\right)+G Q_{x y}^{(3)}\left(\underline{\Phi}_{2}^{(3)}-\underline{\Phi}_{7}^{(3)}\right)\right] \underline{I(0)}^{(3)} \\
& -\left[G J_{\theta x}^{(2)} \underline{\Phi}_{10}^{(2)}-G Q_{x z}^{(2)} \underline{\Phi}_{11}^{(2)}+G Q_{x x}^{(2)}\left(\underline{\Phi}_{4}^{(2)}+\underline{\Phi}_{5}^{(2)}\right)+G Q_{x y}^{(2)}\left(\underline{\Phi}_{2}^{(2)}-\underline{\Phi}_{7}^{(2)}\right)\right]{\underline{I\left(l_{2}\right)}}^{(2)} \\
& F_{G}(27,1)=\left[-E \Gamma_{z x}^{(3)} \underline{\Phi}_{12}^{(3)}+E I_{x x}^{(3)} \underline{\Phi}_{6}^{(3)}-E I_{x y}^{(3)} \underline{\Phi}_{8}^{(3)}\right] \underline{I(0)}^{(3)}-E \Gamma_{z x}^{(2)} \underline{\Phi}_{12}^{(2)}+E I_{x x}^{(2)} \underline{\Phi}_{6}^{(2)} \\
& \left.-E I_{x y}^{(2)} \underline{\Phi}_{8}^{(2)}\right]{\underline{I\left(l_{2}\right)^{(2)}}}^{(0)} \\
& F_{G}(28,1)=\left[E \Gamma_{z y}^{(3)} \underline{\Phi}_{12}^{(3)}+E I_{x y}^{(3)} \underline{\Phi}_{6}^{(3)}-E I_{y y}^{(3)} \underline{\Phi}_{8}^{(3)}\right] \underline{I(0)}^{(3)} \\
& -\left[E \Gamma_{z y}^{(2)} \underline{\Phi}_{12}^{(2)}+E I_{x y}^{(2)} \underline{\Phi}_{6}^{(2)}-E I_{y y}^{(2)} \underline{\Phi}_{8}^{(2)}\right]{\underline{I\left(l_{2}\right)^{(2)}}}^{(2)} \\
& F_{G}(29,1)=\left[G J_{\theta \theta}^{(3)} \underline{\Phi}_{10}^{(3)}-G J_{\theta z}^{(3)} \underline{\Phi}_{11}^{(3)}+G J_{\theta x}^{(3)}\left(\underline{\Phi}_{4}^{(3)}+\underline{\Phi}_{5}^{(3)}\right)+G J_{\theta y}^{(3)}\left(\underline{\Phi}_{2}^{(3)}-\underline{\Phi}_{7}^{(3)}\right)\right] \underline{I(0)}^{(3)} \\
& -\left[G J_{\theta \theta}^{(2)} \underline{\Phi}_{10}^{(2)}-G J_{\theta z}^{(2)} \underline{\Phi}_{11}^{(2)}+G J_{\theta x}^{(2)}\left(\underline{\Phi}_{4}^{(2)}+\underline{\Phi}_{5}^{(2)}\right)+G J_{\theta y}^{(2)}\left(\underline{\Phi}_{2}^{(2)}-\underline{\Phi}_{7}^{(2)}\right)\right]{\underline{I\left(l_{2}\right)^{(2)}}}^{(2)}
\end{aligned}
$$




$$
\begin{aligned}
& F_{G}(30,1)=\left\langle d_{2}\left[-E \Gamma_{z x}^{(3)} \Phi_{12}^{(3)}+E I_{x x}^{(3)} \underline{\Phi}_{6}^{(3)}-E I_{x y}^{(3)} \underline{\Phi}_{8}^{(3)}\right]\right. \\
& +d_{3}\left[E \Gamma_{z y}^{(3)} \underline{\Phi}_{12}^{(3)}+E I_{x y}^{(3)} \Phi_{6}^{(3)}\right. \\
& \left.\left.-E I_{y y}^{(3)}\right]-d_{4}\left[E \Gamma_{z z}^{(3)} \underline{\Phi}_{12}^{(3)}-E \Gamma_{z x}^{(3)} \underline{\Phi}_{6}^{(3)}-E \Gamma_{z y}^{(3)} \underline{\Phi}_{8}^{(3)}\right]\right\rangle \underline{I(0)}^{(3)} \\
& -\left[E \Gamma_{z z}^{(2)} \underline{\Phi}_{12}^{(2)}-E \Gamma_{z x}^{(2)} \underline{\Phi}_{6}^{(2)}-E \Gamma_{z y}^{(2)} \underline{\Phi}_{8}^{(2)}\right] I\left(l_{2}\right)^{(2)} \\
& F_{G}(31,1)=P_{x}-\left[G J_{\theta y}^{(3)} \underline{\Phi}_{10}^{(3)}-G Q_{y z}^{(3)} \underline{\Phi}_{11}^{(3)}+G Q_{x y}^{(3)}\left(\underline{\Phi}_{4}^{(3)}+\underline{\Phi}_{5}^{(3)}\right)\right. \\
& \left.+G Q_{y y}^{(3)}\left(\underline{\Phi}_{2}^{(3)}-\underline{\Phi}_{7}^{(3)}\right)\right]{\underline{I\left(l_{3}\right.}}^{(3)} \\
& F_{G}(32,1)=P_{y}-\left[G J_{\theta x}^{(3)} \underline{\Phi}_{10}^{(3)}-G Q_{x z}^{(3)} \underline{\Phi}_{11}^{(3)}+G Q_{x x}^{(3)}\left(\underline{\Phi}_{4}^{(3)}+\underline{\Phi}_{5}^{(3)}\right)\right. \\
& \left.+G Q_{x y}^{(3)}\left(\underline{\Phi}_{2}^{(3)}-\underline{\Phi}_{7}^{(3)}\right)\right]{\underline{I\left(l_{3}\right.}}^{(3)} \\
& F_{G}(33,1)=M_{x}-E \Gamma_{z x}^{(3)} \underline{\Phi}_{12}^{(3)}+E I_{x x}^{(3)} \underline{\Phi}_{6}^{(3)}-E I_{x y}^{(3)} \underline{\Phi}_{8}^{(3)}{\underline{I\left(l_{3}\right)}}^{(3)} \\
& F_{G}(34,1)=M_{y}-\left[E \Gamma_{z y}^{(3)} \underline{\Phi}_{12}^{(3)}+E I_{x y}^{(3)} \underline{\Phi}_{6}^{(3)}-E I_{y y}^{(3)} \underline{\Phi}_{8}^{(3)}\right]{\underline{I\left(l_{3}\right)^{(3)}}}^{(3)} \\
& \left.F_{G}(35,1)=M_{z}-\left[G J_{\theta \theta}^{(3)} \underline{\Phi}_{10}^{(3)}-G J_{\theta z}^{(3)} \underline{\Phi}_{11}^{(3)}+G J_{\theta x}^{(3)}\left(\underline{\Phi}_{4}^{(3)}+\underline{\Phi}_{5}^{(3)}\right)+G J_{\theta y}^{(3)}\left(\underline{\Phi}_{2}^{(3)}-\underline{\Phi}_{7}^{(3)}\right)\right] \underline{I(l}_{3}\right)^{(3)} \\
& \left.F_{G}(36,1)=-M_{\omega}-\left[E \Gamma_{z z}^{(3)} \underline{\Phi}_{12}^{(3)}-E \Gamma_{z x}^{(3)} \underline{\Phi}_{6}^{(3)}-E \Gamma_{z y}^{(3)} \underline{\Phi}_{8}^{(3)}\right] I \underline{I}_{3}\right)^{(3)}
\end{aligned}
$$


Appendix C Configurations of Shell Model used to validate the results obtained by closed form solution

Software: ABAQUS ${ }^{\circledR}$

Numerical approach: Finite element

Part dimension:

Shape: Rectangular thin-walled cross-section

Cross-section dimension: $0.4 m \times 0.2 m$

Span length: $6 \mathrm{~m}$

Element type: Shell element, $S 4 R$

Material:

Type: Mechanical

Elasticity type: Elastic

Elastic type: Isotropic

Young's modulus: $71.7 \times 10^{9} \mathrm{~Pa}$

Poisson's ratio: 0.33

Section: Solid, homogeneous, shell thickness: $0.01 \mathrm{~m}$

Mesh size: $0.05 \mathrm{~m}$

Analysis:

Static response (deformation)

Step:

Procedure type: General, Static

Incrementation: $1 \times 10^{-5}$ of the full load

Dynamic response (frequency)

Step:

Procedure type: Linear perturbation, Frequency

Eigensolver: Lanzos 


\section{References}




\section{References}

[1] Nguyen, N., et al., "Dynamics and Adaptive Control for Stability Recovery of Damaged Asymmetric Aircraft," pp. 169-191, Vol. 1, Collection of Technical Papers - AIAA Guidance, Navigation and Control Conference 2006. Edited. American Institute of Aeronautics and Astronautics Inc., Keystone, CO, USA, 2006.

[2] Megson, T.H.G., Aircraft Structures for Engineering Students, Butterworth-Heinemann, MA, 2007.

[3] Timoshenko, S.P., History of Strength of Materials: With a Brief Account of the History of Theory of Elasticity and Theory of Structures, Dover Publications, New York, NY, 1983.

[4] Murray, N.W., Introduction to the Theory of Thin-Walled Structures, Clarendon Press, Oxford, 1984.

[5] Gjelsvik, A., The Theory of Thin Walled Bars, Wiley, New York, NY, 1981.

[6] Timoshenko, S.P., Theory of Elastic Stability, McGraw-Hill, New York, NY, 1961.

[7] Vlasov, V.Z., Thin-Walled Elastic Beams, National Science Foundation, Washington DC, 1961.

[8] Benscoter, S.U., "Theory of Torsion Bending for Multicell Beams," Journal of Applied Mechanics, Transactions of the ASME, Vol. 21, No.1, 1954, pp. 25-34.

[9] Nishino, F., Hasegawa, A., and Natori, E., "Thin-Walled Rectangular Beams with Shear Deformation and Cross Sectional Distortion," Mechanics in Engineering, ASCE-EMD, Vol. 11, 1977, pp. 183-206.

[10] Bishop, R.E.D. and Price, W.G., "Coupled Bending and Twisting of a Timoshenko Beam," Journal of Sound and Vibration, Vol. 50, No.4, 1977, pp. 469-477.

[11] Capuani, D., Savoia, M., and Laudiero, F., "A Generalization of the Timoshenko Beam Model for Coupled Vibration Analysis of Thin-Walled Beams," Earthquake Engineering \& Structural Dynamics, Vol. 21, No.10, 1992, pp. 859-879.

[12] Bercin, A.N. and Tanaka, M., "Coupled Flexural-Torsional Vibrations of Timoshenko Beams," Journal of Sound and Vibration, Vol. 207, No.1, 1997, pp. 47-59.

[13] Shakourzadeh, H., Guo, Y.Q., and Batoz, J.L., "A Torsion Bending Element for ThinWalled Beams with Open and Closed Cross Sections," Computers \& Structures, Vol. 55, No.6, 1995, pp. 1045-1054. 
[14] Dokumaci, E., "An Exact Solution for Coupled Bending and Torsion Vibrations of Uniform Beams Having Single Cross-Sectional Symmetry," Journal of Sound and Vibration, Vol. 119, No.3, 1987, pp. 443-449.

[15] Falco, M. and Gasparetto, M., "Flexural-Torsional Vibrations of Thin-Walled Beams," Meccanica, Vol. 8, No.3, 1973, pp. 181-189.

[16] Bishop, R.E.D., Cannon, S.M., and Miao, S., "On Coupled Bending and Torsional Vibration of Uniform Beams," Journal of Sound and Vibration, Vol. 131, No.3, 1989, pp. 457-464.

[17] Prokic, A., "Thin-Walled Beams with Open and Closed Cross-Sections," Computers \& Structures, Vol. 47, No.6, 1993, pp. 1065-1070.

[18] Gunnlaugsson, G.A. and Pedersen, P.T., "A Finite Element Formulation for Beams with Thin Walled Cross-Sections," Computers and Structures, Vol. 15, No.6, 1982, pp. 691-699.

[19] Tralli, A., "A Simple Hybrid Model for Torsion and Flexure of Thin-Walled Beams," Computers \& Structures, Vol. 22, No.4, 1986, pp. 649-658.

[20] Pedersen, P.T., "Beam Theories for Torsional-Bending Response of Ship Hulls," Journal of Ship Research, Vol. 35, No.3, 1991, pp. 254-265.

[21] Capurso, M., "Sur Calcolo Delle Travi Di Parete Sotlite in Presenta De Forze E Distorsions," La Ricerca Scientifico, Vol. 6, 1964, pp. 213-286.

[22] Librescu, L. and Song, O., Thin-Walled Composite Beams: Theory and Application, Springer, Dordrecht, The Netherlands, 2006.

[23] Prokic, A., "New Warping Function for Thin-Walled Beams 2: Finite Element Method and Applications," Journal of Structural Engineering, Vol. 122, No.12, 1996, pp. 1443-1451.

[24] Prokic, A., "New Warping Function for Thin-Walled Beams 1: Theory," Journal of Structural Engineering, Vol. 122, No.12, 1996, pp. 1437-1442.

[25] Fujii, D. and Park, S.-W., "Torsional Analysis of Thin-Walled Beam with Discontinuously Variable Cross-Section by Using Shear Warping Function," Journal of Structural and Construction Engineering, Transactions of the AIJ, Vol. 487, 1996, pp. 93-101.

[26] Park, S.-W., Fujii, D., and Fujitani, Y., "A Finite Element Analysis of Discontinuous ThinWalled Beams Considering Nonuniform Shear Warping Deformation," Computers \& Structures, Vol. 65, No.1, 1997, pp. 17-27. 
[27] Saade, K., Espion, B., and Warzee, G., "Non-Uniform Torsional Behavior and Stability of Thin-Walled Elastic Beams with Arbitrary Cross Sections," Thin-Walled Structures, Vol. 42, No.6, 2004, pp. 857-881.

[28] Timoshenko, S.P., Vibration Problems in Engineering, D. Van Nostrand Company, New York, NY, 1937.

[29] Meirovitch, L., Elements of Vibration Analysis, McGraw-Hill, New York, NY, 1975.

[30] Gere, J.M., "Torsional Vibrations of Beams of Thin-Walled Open Section," Journal of Applied Mechanics,Transactions of the ASME, Vol. 21, No.4, 1954, pp. 381-387.

[31] Gere, J.M. and Lin, Y.K., "Coupled Vibrations of Thin-Walled Beams of Open Cross Section," Journal of Applied Mechanics,Transactions of the ASME, Vol. 25, 1957, pp. 373-378. [32] Hallauer, W.L. and Liu, R.Y.L., "Beam Bending-Torsion Dynamic Stiffness Method for Calculation of Exact Vibration Modes," Journal of Sound and Vibration, Vol. 85, No.1, 1982, pp. 105-113.

[33] Rozmarynowski, B. and Szymczak, C., "Non-Linear Free Torsional Vibrations of ThinWalled Beams with Bisymmetric Cross-Section," Journal of Sound and Vibration, Vol. 97, No.1, 1984, pp. 145-53.

[34] Noor, A.K., Peters, J.M., and Min, B.-J., "Mixed Finite Element Models for Free Vibrations of Thin-Walled Beams," Finite Elements in Analysis and Design, Vol. 5, No.4, 1989, pp. 291305.

[35] Ahmad, F.M. and Guile, C.W., "Analysis of Coupled Natural Frequencies of Thin-Walled Beams with Opens Cross Section Using Msc/Nastran," The MSC 1990 World Users Conference Proceedings, Vol. 1, No.15, 1990, pp. 1-9.

[36] Banerjee, J.R. and Williams, F.W., "Coupled Bending-Torsional Dynamic Stiffness Matrix for Timoshenko Beam Elements," Computers \& Structures, Vol. 42, No.3, 1992, pp. 301-310.

[37] Mcgee, O.G., Owings, M.I., and Harris, J.W., "Torsional Vibration of Pretwisted ThinWalled Cantilevered I-Beams," Computers \& Structures, Vol. 47, No.1, 1993, pp. 47-56.

[38] Eisenberger, M., "Torsional Vibrations of Open and Variable Cross Section Bars," ThinWalled Structures, Vol. 28, No.3-4, 1997, pp. 269-278.

[39] Wu, J.J. and Whittaker, A.R., "The Natural Frequencies and Mode Shapes of a Uniform Cantilever Beam with Multiple Two-Dof Spring-Mass Systems," Journal of Sound and Vibration, Vol. 227, No.2, 1999, pp. 361-381. 
[40] Kuang, J.S. and Ng, S.C., "Coupled Lateral-Torsion Vibration of Asymmetric Shear-Wall Structures," Thin-Walled Structures, Vol. 38, No.2, 2000, pp. 93-104.

[41] Ambrosini, D. and Danesi, R., "Free Vibration of Nonsymmetrical Thin Walled Beams," Mecánica Computacional, Vol. 22, 2003, pp. 666-677.

[42] Mohri, F., Azrar, L., and Potier-Ferry, M., "Vibration Analysis of Buckled Thin-Walled Beams with Open Sections," Journal of Sound and Vibration, Vol. 275, No.1-2, 2004, pp. 434446.

[43] Prokic, A., "On Triply Coupled Vibrations of Thin-Walled Beams with Arbitrary CrossSection," Journal of Sound and Vibration, Vol. 279, No.3-5, 2005, pp. 723-737.

[44] Prokic, A., "On Fivefold Coupled Vibrations of Timoshenko Thin-Walled Beams," Engineering Structures, Vol. 28, No.1, 2006, pp. 54-62.

[45] Kapania, R. and Kim, Y.-Y., "Flexural-Torsional Coupled Vibration of Slewing Beams Using Various Types of Orthogonal Polynomials," Journal of Mechanical Science and Technology, Vol. 20, No.11, 2006, pp. 1790-1800.

[46] Senjanovic, I., Catipovic, I., and Tomasevic, S., "Coupled Flexural and Torsional Vibrations of Ship-Like Girders," Thin-Walled Structures, Vol. 45, No.12, 2007, pp. 1002-1021.

[47] Song, O. and Librescu, L., "Bending Vibration of Cantilevered Thin-Walled Beams Subjected to Time-Dependent External Excitations," The Journal of the Acoustical Society of America, Vol. 98, No.1, 1995, pp. 313-319.

[48] Librescu, L. and Na, S., "Dynamic Response of Cantilevered Thin-Walled Beams to Blast and Sonic-Boom Loadings," Shock and Vibration, Vol. 5, No.1, 1998, pp. 23-33.

[49] Suresh, R. and Malhotra, S.K., "Vibration and Damping Analysis of Thin-Walled Box Beams," Journal of Sound and Vibration, Vol. 215, No.2, 1998, pp. 201-210.

[50] Qin, Z., "Vibration and Aeroelasticity of Advanced Aircraft Wings Modeled as Thin-Walled Beams - Dynamics, Stability and Control," Virginia Polytechnic Institute and State University, Blacksburg, VA, 2001.

[51] Librescu, L.I. and Na, S., "Vibration Control of Adaptive Aircraft Wings Modeled as Tapered Thin-Walled Beams," pp. 319-330in Smart Structures and Materials 2001: Modeling, Signal Processing, and Control in Smart Structures, Vol. 4326. Edited. SPIE, Newport Beach, CA, 2001. 
[52] Jun, L., et al., "Bending-Torsional Coupled Dynamic Response of Axially Loaded Composite Timosenko Thin-Walled Beam with Closed Cross-Section," Composite Structures, Vol. 64, No.1, 2004, pp. 23-35.

[53] Wang, K., "Vibration Analysis of Cracked Composite Bending-Torsion Beams for Damage Diagnosis," Virginia Polytechnic Institute and State University, Blacksburg, VA, 2004.

[54] Freudenthal, A.M., "Safety and Probability of Structural Failure," Journal of the Structural Division, Transactions of the ASCE, Vol. 80, No.468, 1954, pp. 1337-1397.

[55] Freudenthal, A.M., "Safety, Reliability and Structural Design," Journal of the Structural Division, Transactions of the ASCE, Vol. 87, No.ST3, Part 1, 1961, pp. 1-16.

[56] Freudenthal, A.M., Garrelts, J.M., and Shinozuka, M., "The Analysis of Structural Safety," Journal of the Structural Division, Transactions of the ASCE, Vol. 92, No.ST1, 1966, pp. 267325.

[57] Cornell, C.A., "Bounds on the Reliability of Structural Systems," Journal of Structural Division, ASCE, Vol. 93, No.1, 1967, pp. 171-200.

[58] Cornell, C.A., Structural Safety Specifications Based on Second-Moment Reliability Analysis, London, 1969.

[59] Ang, A.H.-S. and Cornell, C.A., "Reliability Bases of Structural Safety and Design," Journal of the Structural Division, Transactions of the ASCE, Vol. 100, No.ST9, 1974, pp. 17551769.

[60] Hasofer, A.M. and Lind, N.C., "Exact and Invariant Second-Moment Code Format," 100, No.EM1, 1974, pp. 111-121.

[61] Rackwitz, R., Practical Probabilistic Approach to Design, Munich, Germany, 1976.

[62] Rackwitz, R. and Fiessler, B., Note on Discrete Safety Checking When Using Non-Normal Stochastic Models for Basic Variables, MIT, Cambridge, MA, 1976.

[63] Rackwitz, R. and Fiessler, B., "Structural Reliability under Combined Random Load Sequences," Computers and Structures, Vol. 9, No.5, 1978, pp. 489-494.

[64] Haldar, A. and Mahadevan, S., Probability, Reliability and Statistical Methods in Engineering Design, Wiley, New York, 2000.

[65] Fiessler, B., Neumann, H.-J., and Rackwitz, R., "Quadratic Limit States in Structural Reliability," 105, No.4, 1979, pp. 661-676. 
[66] Ang, A.H.-S. and Tang, W.H., Probability Concepts in Engineering Planning and Design: Volume 2 - Decision, Risk and Reliability, Vol. 2, Wiley, New York, NY, 1984.

[67] Elishakoff, I., Probabilistic Methods in the Theory of Structures, Wiley, New York, 1983.

[68] Melchers, R.E., Structural Reliability: Analysis and Prediction, Ellis Horwood ,Wiley, Chichester, W. Sussex, England, 1987.

[69] Rao, S.S., Reliability-Based Design, McGraw-Hill, New York, NY, 1992.

[70] Nowak, A.S. and Collins, K.R., Reliability of Structures, McGraw-Hill, Boston, MA, 2000.

[71] Nikolaidis, E., Ghiocel, D.M., and Singhal, S., Engineering Design Reliability Handbook, CRC Press, Boca Raton, 2005.

[72] Nikolaidis, E., Ghiocel, D.M., and Singhal, S., Engineering Design Reliability Applications for the Aerospace, Automotive, and Ship Industries, CRC Press, Boca Raton, 2008.

[73] Choi, S.-K., Grandhi, R.V., and Canfield, R.A., Reliability-Based Structural Design, Springer, London, 2007.

[74] Maymon, G., "Probability of Failure of Structures without a Closed-Form Failure Function," Computers \& Structures, Vol. 49, No.2, 1993, pp. 301-313.

[75] Lin, Y.K. and Cai, G.Q., Probabilistic Structural Dynamics : Advanced Theory and Applications, McGraw-Hill, New York, 2004.

[76] Crandall, S.H., Random Vibration, Technology Press of the Massachusetts Institute of Technology, Cambridge, MA, 1959.

[77] Crandall, S.H. and Mark, W.D., Random Vibration in Mechanical Systems, Academic Press, New York, NY, 1963.

[78] Lin, Y.K., Probabilistic Theory of Structural Dynamics, McGraw-Hill, New York, 1967.

[79] Shinozuka, M. and Yang, J.N., "Random Vibration of Linear Structures," International Journal of Solids and Structures, Vol. 5, No.10, 1969, pp. 1005-1036.

[80] Davies, H.G., "Random Vibration of a Beam Impacting Stops," Journal of Sound and Vibration, Vol. 68, No.4, 1980, pp. 479-487.

[81] Elishakoff, I. and Livshits, D., "Some Closed-Form Solutions in Random Vibration of Bernoulli-Euler Beams," International Journal of Engineering Science, Vol. 22, No.11-12, 1984, pp. 1291-1301. 
[82] Elishakoff, I. and Livshits, D., "Some Closed-Form Solutions in Random Vibration of Bresse-Timoshenko Beams," Probabilistic Engineering Mechanics, Vol. 4, No.1, 1989, pp. 4954.

[83] Singh, M.P. and Abdelnaser, A.S., "Random Vibrations of Externally Damped Viscoelastic Timoshenko Beams with General Boundary Conditions," Journal of Applied Mechanics,Transactions of the ASME, Vol. 60, No.1, 1993, pp. 149-156.

[84] Abdelnaser, A.S. and Singh, M.P., "Random Vibrations of Cantilevered Composite Beams with Torsion-Bending Coupling," Probabilistic Engineering Mechanics, Vol. 8, No.3-4, 1993, pp. 143-151.

[85] Eslimy-Isfahany, S.H.R., Banerjee, J.R., and Sobey, A.J., "Response of a Bending-Torsion Coupled Beam to Deterministic and Random Loads," Journal of Sound and Vibration, Vol. 195, No.2, 1996, pp. 267-283.

[86] Jun, L., et al., "Response of Monosymmetric Thin-Walled Timoshenko Beams to Random Excitations," International Journal of Solids and Structures, Vol. 41, No.22-23, 2004, pp. 60236040 .

[87] Jun, L. and Xianding, J., "Response of Flexure-Torsion Coupled Composite Thin-Walled Beams with Closed Cross-Sections to Random Loads," Mechanics Research Communications, Vol. 32, No.1, 2005, pp. 25-41.

[88] Nigam, N.C., Introduction to Random Vibrations, MIT Press, Cambridge, MA, 1983.

[89] Nigam, N.C. and Narayanan, S., Applications of Random Vibrations, Springer-Verlag, Narosa Publishing House, New York, NY, 1994.

[90] Newland, D.E., An Introduction to Random Vibrations, Spectral and Wavelet Analysis, Longman Scientific \& Technical, Wiley, Harlow, Essex, England, 1993.

[91] Lutes, L.D. and Sarkani, S., Random Vibrations: Analysis of Structural and Mechanical Systems, Butterworth-Heinemann, Oxford, 2004.

[92] Soong, T.T. and Grigoriu, M., Random Vibration of Mechanical and Structural Systems, PTR Prentice Hall, Englewood Cliffs, NJ, 1993.

[93] Heller, A.S., Heller, R.A., and Freudenthal, A.M., "Random Fatigue Failure of a Multiple Load Path Redundant Structure," pp. 592-611 Edited, United States, 1944. 
[94] Freudenthal, A.M., "The Statistical Aspect of Fatigue of Materials," Proceedings of the Royal Society of London, Series A (Mathematical and Physical Sciences), Vol. 187, 1946, pp. 416-429.

[95] Freudenthal, A.M. and Gumbel, E.J., "On the Statistical Interpretation of Fatigue Tests," Proceedings of the Royal Society of London, Series A (Mathematical and Physical Sciences), Vol. 216, 1953, pp. 309-332.

[96] Heller, R.A., Probabilistic Aspects of Fatigue, Vol. 511, American Society for Testing and Materials, Philadelphia, PA, 1972.

[97] Haugen, E.B., Probabilistic Mechanical Design, Wiley, New York, NY, 1980.

[98] Provan, J.W., Probabilistic Fracture Mechanics and Reliability, M. Nijhoff, Dordrecht, The Netherlands, 1987.

[99] Sobczyk, K. and Spencer, B.F., Random Fatigue: From Data to Theory, Academic Press, Boston, MA, 1992.

[100] Schijve, J., Fatigue of Structures and Materials, Springer, Dordrecht, The Netherlands, 2008.

[101] Castillo, E. and Fernández-Canteli, A., A Unified Statistical Methodology for Modeling Fatigue Damage, Springer, Dordrecht, The Netherlands, 2009.

[102] Puatatsananon, W. and Saouma, V.E., "Reliability Analysis in Fracture Mechanics Using the First-Order Reliability Method and Monte Carlo Simulation," Fatigue \& Fracture of Engineering Materials \& Structures, Vol. 29, No.11, 2006, pp. 959-975.

[103] Paris, P. and Erdogan, F., "A Critical Analysis of Crack Propagation Laws," Journal of Basic Engineering,Transactions of the ASME, Vol. 85, No.4, 1963, pp. 528-534.

[104] Forman, R.G., Kearney, V.E., and Engle, R.M., "Numerical Analysis of Crack Propagation in Cyclic-Loaded Structures," Journal of Basic Engineering, Transactions of the ASME Series D, Vol. 89, No.3, 1967, pp. 459-464.

[105] Walker, E.K., The Effect of Stress Ratio During Crack Propagation and Fatigue for 2024T3 and 7076-T6 Aluminum, Effect of Environment and Complex Load History on Fatigue Life, Vol. 462, American Society for Testing and Materials, Philadelphia, PA, 1970.

[106] Bannantine, J.A., Comer, J.J., and Handrock, J.L., Fundamentals of Metal Fatigue Analysis, Prentice Hall, Englewood Cliffs, NJ, 1990. 
[107] Skelton, R.P., "The Prediction of Crack Growth Rates from Total Endurances in High Strain Fatigue," Fatigue of Engineering Materials and Structures, Vol. 2, No.3, 1979, pp. 305318.

[108] Wanhill, R.J.H., "Gust Spectrum Fatigue Crack Propagation in Candidate Skin Materials," Fatigue of Engineering Materials and Structures, Vol. 1, No.1, 1979, pp. 5-19.

[109] Kujawski, D. and Ellyin, F., "A Fatigue Crack Propagation Model," Engineering Fracture Mechanics, Vol. 20, No.5-6, 1984, pp. 695-704.

[110] Ellyin, F. and Kujawski, D., "Plastic Strain Energy in Fatigue Failure," Journal of Pressure Vessel Technology, Transactions of the ASME, Vol. 106, No.4, 1984, pp. 342-347.

[111] Rahman, S., Wilkowski, G., and Mohan, R., "Low-Cycle Fatigue Crack Growth Considerations in Pipe Fracture Analyses," Nuclear Engineering and Design, Vol. 168, No.1-3, 1997, pp. 105-118.

[112] Beden, S.M., Abdullah, S., and Ariffin, A.K., "Review of Fatigue Crack Propagation Models for Metallic Components," European Journal of Scientific Research, Vol. 28, No.3, 2009, pp. 364-397.

[113] Virkler, D.A., Hillberry, B.M., and Goel, P.K., "The Statistical Nature of Fatigue Crack Propagation," Journal of Engineering Materials and Technology, Transactions of the ASME, Vol. 101, No.2, 1979, pp. 148-153.

[114] Yang, J.N. and Donath, R.C., "Statistical Crack Propagation in Fastener Holes under Spectrum Loading," Journal of Aircraft, Vol. 20, No.12, 1983, pp. 1028-1032.

[115] Yang, J.N., Hsi, W.H., Manning, S. D., and Rudd, J.L., "Stochastic Crack Propagation in Fastener Holes," Journal of Aircraft, Vol. 22, No.9, 1985, pp. 810-817.

[116] Lin, Y.K. and Yang, J.N., "Stochastic Theory of Fatigue Crack Propagation," AIAA Journal, Vol. 23, No.1, 1985, pp. 117-124.

[117] Oh, K.P., "A Diffusion Model for Fatigue Crack Growth," Proceedings of the Royal Society of London, Series A (Mathematical and Physical Sciences), Vol. 367, No.1728, 1979, pp. 47-58.

[118] Sobczyk, K., "Markov Models for Fatigue Crack Growth," Engineering Fracture Mechanics, Vol. 31, No.4, 1988, pp. 713-714.

[119] Ditlevsen, O. and Sobczyk, K., "Random Fatigue Crack Growth with Retardation," Engineering Fracture Mechanics, Vol. 24, No.6, 1986, pp. 861-878. 
[120] Liu, W.K., et al., "Three Reliability Methods for Fatigue Crack Growth," Engineering Fracture Mechanics, Vol. 53, No.5, 1996, pp. 733-752.

[121] Bloom, J.M. and Ekvall, J.C., Probabilistic Fracture Mechanics and Fatigue Methods: Applications for Structural Design and Maintenance, American Society for Testing and Materials, Philadelphia, PA, 1983.

[122] Ellyin, F. and Fakinlede, C.O., "Probabilistic Simulation of Fatigue Crack Growth by Damage Accumulation," Engineering Fracture Mechanics, Vol. 22, No.4, 1985, pp. 697-712. [123] Bogdanoff, J.L. and Kozin, F., Probabilistic Models of Cumulative Damage, Wiley, New York, NY, 1985.

[124] Ellyin, F., Fatigue Damage, Crack Growth, and Life Prediction, Chapman \& Hall, London, 1997.

[125] Maymon, G., Some Engineering Applications in Random Vibrations and Random Structures, American Institute of Aeronautics and Astronautics, Reston, VA, 1998.

[126] Maymon, G., Structural Dynamics and Probabilistic Analysis for Engineers, ButterworthHeinemann, Burlington, 2008.

[127] Kunaporn, C., et al., "Probabilistic Analysis of a Thin-Walled Beam with a Crack," in the 51st AIAA/ASME/ASCE/AHS/ASC Structures, Structural Dynamics, and Materials Conference. Edited. American Institute of Aeronautics and Astronautics, Orlando, Fl, 2010.

[128] Kunaporn, C., et al., "Random Crack Propagation Analysis of a Beam with a Crack," in the 51st AIAA/ASME/ASCE/AHS/ASC Structures, Structural Dynamics, and Materials Conference. Edited. American Institute of Aeronautics and Astronautics, Orlando, Fl, 2010.

[129] Dvorkin, E.N., et al., "A Vlasov Beam Element," Computers \& Structures, Vol. 33, No.1, 1989, pp. 187-196.

[130] Pedersen, P.T., "A Beam Model for the Torsional-Bending Response of Ship Hulls," Transaction of Royal Institution of Naval Architects, Vol. 125, 1982, pp. 171-182.

[131] Prokic, A. and Lukic, D., "Dynamic Analysis of Thin-Walled Closed-Section Beams," Journal of Sound and Vibration, Vol. 302, No.4-5, 2007, pp. 962-980.

[132] Weintraub, S.H., Jordan Canonical Form : Theory and Practice, Morgan \& Claypool Publishers, San Rafael, CA, 2009.

[133] Dang, T.D., Kapania, R.K., and Patil, M.J., "Analytical Modeling of Cracked Thin-Walled Beams under Torsion," AIAA Journal, Vol. 48, No.3, 2010, pp. 664-75. 
[134] Rao, S.S., Mechanical Vibrations, Addison-Wesley, Reading, MA, 1995.

[135] Yang, T.Y. and Kapania, R.K., "Finite Element Random Response Analysis of Cooling Tower," Journal of Engineering Mechanics, Vol. 110, No.4, 1984, pp. 589-609.

[136] Leadbetter, M.R., Lindgren, G., and Rootzén, H., Extremes and Related Properties of Random Sequences and Processes, Springer-Verlag, New York, NY, 1983.

[137] Sanford, R.J., Principles of Fracture Mechanics, Prentice Hall, Upper Saddle River, NJ, 2003.

[138] Wright, J.R. and Cooper, J.E., Introduction to Aircraft Aeroelasticity and Loads, John Wiley, Hoboken, NJ, 2007.

[139] Howe, D., Aircraft Loading and Structural Layout, American Institute of Aeronautics and Astronautics, Reston, VA, 2004.

[140] Dowell, E.H., et al., A Modern Course in Aeroelasticity, Kluwer Academic Publishers, Boston, MA, 2004. 